\title{
Rough nonlocal diffusions
}

\author{
Michele Coghi ${ }^{*}$ Torstein Nilssen ${ }^{\dagger}$
}

\begin{abstract}
We consider a nonlinear Fokker-Planck equation driven by a deterministic rough path which describes the conditional probability of a McKean-Vlasov diffusion with "common" noise. To study the equation we build a self-contained framework of non-linear rough integration theory which we use to study McKean-Vlasov equations perturbed by rough paths. We construct an appropriate notion of solution of the corresponding Fokker-Planck equation and prove wellposedness.
\end{abstract}

MSC Classification Numbers: 60H05, 60H15, 60J60, 35K55.

Key words: Rough paths, Stochastic PDEs, McKean-Vlasov, non-local equations.

\section{Contents}

1 Introduction

2 Notations and preliminary results 6

2.1 Hölder and p-variation spaces . . . . . . . . . . . . . . . . 6

2.2 Rough paths . . . . . . . . . . . . . . . . . . . 7

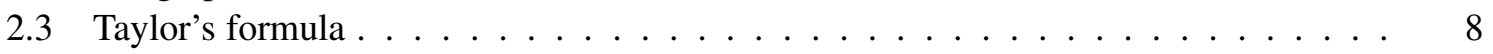

2.4 Wasserstein metric . . . . . . . . . . . . . . . . . . . . . . . . . 9

2.5 Spatial function spaces . . . . . . . . . . . . . . . . . 9

3 Non linear integration $\quad 10$

3.1 A priori estimates . . . . . . . . . . . . . . . . . 12

3.2 A priori contractive estimates . . . . . . . . . . . . . . . 14

3.3 Well-posedness of nonlinear RDEs . . . . . . . . . . . . . . . 18

4 Rough non-linearities $\quad 19$

4.1 Construction of the rough driver . . . . . . . . . . . . . . . 20

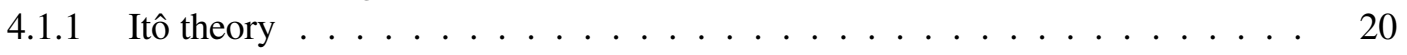

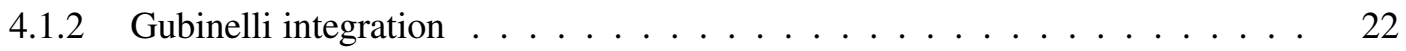

4.1.3 Mixed Itô and rough path integration . . . . . . . . . . . 23

4.2 Integrability of the random rough driver . . . . . . . . . . . . . 25

4.3 The average Itô formula . . . . . . . . . . . . . . . . . 28

${ }^{*}$ WIAS Berlin, Mohrenstraße 39, 10117 Berlin. Support from the Berlin Mathematics Research Center MATH+ is gratefully acknowledged.

${ }^{\dagger}$ Institute of Mathematics, Technical University of Berlin, Germany, Financial support by the DFG via Research Unit FOR 2402 is gratefully acknowledged. 
5 Linear Rough PDE 34

5.1 Unbounded rough drivers . . . . . . . . . . . . . . . . . . . . . . . . . . . .

5.2 A priori estimates for smooth vector fields . . . . . . . . . . . . . 38

5.3 Existence of a smooth solution . . . . . . . . . . . . . . . . . . 42

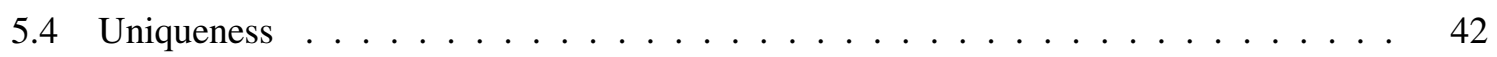

6 The McKean-Vlasov equation 43

7 Non local rough PDEs 47

A Appendix 49

A.1 Kolmogorov continuity theorem . . . . . . . . . . . . . . . . . . 49

A.2 Weakly geometric rough paths . . . . . . . . . . . . . . . 50

A.3 A separable subspace of the Hölder space . . . . . . . . . . . . . 52 52

\section{Introduction}

The term diffusion is sometimes used interchangeably when talking either about the macroscopic (Eulerian) description of the density of a substance occupying some space or the infinitesimal (Lagrangian) description of the particles of the substance. Many physical phenomena are however inherently nonlinear in the sense that the dynamic of the system will depend not only on space but also on the density of the substance itself. In this paper we study this type of nonlinear diffusion from both the Eulerian and Lagrangian perspective when the diffusion is perturbed by a rough path. We are motivated by dynamics that arise from interacting particle systems with common noise;

$$
d X_{t}^{i}=\frac{1}{N} \sum_{j=1}^{N} b\left(X_{t}^{j}, X_{t}^{i}\right) d t+\frac{1}{N} \sum_{j=1}^{N} \sigma\left(X_{t}^{j}, X_{t}^{i}\right) d W_{t}^{i}+\frac{1}{N} \sum_{j=1}^{N} \beta\left(X_{t}^{j}, X_{t}^{i}\right) \circ d B_{t} .
$$

Here each particle $X^{i}$ is influenced by 2 independent sources of noise, the Brownian motion $B \mathbb{1}$ is visible for all particles (common noise) and the Brownian motion $W^{i}$ represents a noise term specific for particle $X^{i}$. Since $B$ is influencing every particle, taking the limit $N \rightarrow \infty$ will only average out the individual noise terms, giving, at least formally, the mean-field dynamics

$$
\begin{cases}d x_{t} & =\int_{\mathbb{R}^{d}} b\left(\omega, x_{t}\right) d \mu_{t}(\omega) d t+\int_{\mathbb{R}^{d}} \sigma\left(\omega, x_{t}\right) d \mu_{t}(\omega) d W_{t}+\int_{\mathbb{R}^{d}} \beta\left(\omega, x_{t}\right) d \mu_{t}(\omega) \circ d B_{t} \\ \mu_{t} & =\mathcal{L}\left(x_{t} \mid \mathcal{F}_{t}^{B}\right) .\end{cases}
$$

We note that the conditional law $\mathcal{L}\left(x_{t} \mid \mathcal{F}_{t}^{B}\right)$ heuristically satisfies the non-local Fokker-Plank equation

$$
d \mu_{t}=\frac{1}{2} \operatorname{Tr} \nabla^{2}\left(\sigma(\mu, \cdot)_{t} \sigma(\mu, \cdot)_{t}^{T} \mu_{t}\right) d t-\operatorname{div}\left(b(\mu, \cdot)_{t} \mu_{t}\right) d t-\operatorname{div}\left(\beta(\mu, \cdot)_{t} \mu_{t}\right) \circ d B_{t}
$$

where we have used the notation $\sigma(\mu, x)_{t}=\int_{\mathbb{R}^{d}} \sigma(\omega, x) d \mu_{t}(\omega)$ etc. and $\operatorname{Tr} \nabla^{2}(a)=\sum_{i, j=1}^{d} \partial_{i} \partial_{j} a^{i, j}$ for a matrix valued function $a$. In fact, we can also address the case when $\sigma$ is a certain type of Lipschitz nonlinearity on $\mathcal{P}\left(\mathbb{R}^{d}\right) \times \mathbb{R}^{d}$, where $\mathcal{P}\left(\mathbb{R}^{d}\right)$ denotes the set of probability measures on $\mathbb{R}^{d}$, see Assumption 6.2. We will only address the case when $\beta$ and $b$ are linear in their second argument.

\footnotetext{
${ }^{1}$ Since we will in this paper only consider geometric rough paths, we shall consider Stratonovich integration for this term.
} 
In practice, (2) is difficult to solve since it needs to be formulated on a very large state space, namely $\left[C\left([0, T] ; \mathcal{P}\left(\mathbb{R}^{d}\right)\right)\right]^{\Omega}$ where $\Omega$ is the underlying probability space. Even when $\Omega$ is finite, this space is too large to do analysis since it is difficult to find compact subsets that is used for proving well-posedness of (1) and (2). For a long time, well-posedness for equation (2) was known only for densities, see [20]. A proper well-posedness result in the space of measures was obtain just very recently in [8].

In this paper we take a different approach, namely we study equation (1) for a fixed sample path of the Brownian motion. Our method relies on the theory of rough paths and as such, allows the study of (1) where $B$ is replaced by any path that can be lifted to a rough path. In particular, no markovianity or martingale structure is needed for the common noise.

From now on we replace $B$ by a (deterministic) rough path $\mathbf{Z}=(Z, \mathbb{Z})$, and equation (2) becomes

$$
\partial_{t} \mu=\frac{1}{2} \operatorname{Tr} \nabla^{2}\left(\sigma(\mu, \cdot) \sigma(\mu, \cdot)^{T} \mu\right)-\operatorname{div}(b(\mu, \cdot) \mu)-\operatorname{div}(\beta(\mu, \cdot) \mu) \dot{\mathbf{Z}} .
$$

The main contribution of this paper is the following.

Theorem (see Theorems 7.2 and7.4). Given a probability measure $\mu_{0}$ on $\mathbb{R}^{d}$ with finite $\rho$-th moment, for any $\rho \geq 2$, there exists a unique measure-valued path $\mu:[0, T] \rightarrow \mathcal{P}\left(\mathbb{R}^{d}\right)$, which solves (3) with initial condition $\mu_{0}$.

Moreover we will prove in Theorem 7.2 that the unique solution is given as $\mu_{t}:=\mathcal{L}\left(x_{t}\right)$, namely the law of solution $x$ to the McKean-Vlasov equation

$$
d x_{t}=b\left(\mathcal{L}\left(x_{t}\right), x_{t}\right) d t+\sigma\left(\mathcal{L}\left(x_{t}\right), x_{t}\right) d W_{t}+\beta\left(\mathcal{L}\left(x_{t}\right), x_{t}\right) d \mathbf{Z}_{t} .
$$

We will show well-posedness of (4) in Section 6 ,

The strategy to prove uniqueness to equation (3) relies on showing that every solution must be the law of the McKean-Vlasov equation. As it will be clear in the proof of Theorem 7.4, this also necessitates to be able to have well-posedness of the equation

$$
d x_{t}=b_{t}\left(x_{t}\right) d t+\sigma_{t}\left(x_{t}\right) d W_{t}+\beta_{t}\left(x_{t}\right) d \mathbf{Z}_{t}
$$

for given time inhomogeneous functions $b, \sigma$ and $\beta$, where the time dependence is induced by the law. Moreover, a common approach to proving well-posedness of (4) is to construct the solution as a fixed point in the space of measures on an appropriate function space. Towards this end one would e.g. define inductively

$$
d x_{t}^{n+1}=b\left(\mathcal{L}\left(x_{t}^{n}\right), x_{t}^{n+1}\right) d t+\sigma\left(\mathcal{L}\left(x_{t}^{n}\right), x_{t}^{n+1}\right) d W_{t}+\beta\left(\mathcal{L}\left(x_{t}^{n}\right), x_{t}^{n+1}\right) d \mathbf{Z}_{t} .
$$

Once again, it is necessary to give a meaning to equation (5). If we consider the case $b=\sigma=0$ and $\beta_{t}(x)=\beta_{t}$ the equation reads

$$
d x_{t}=\beta_{t} d \mathbf{Z}_{t} .
$$

It is well-known that the above integration does not make sense unless we impose additional structure on $\beta$, namely that there exists a Taylor-type expansion around the irregular path $Z$, which is exactly the notion of controlled rough paths as introduced by Gubinelli in [17]. If one aims to solve a mean-field equation on the form

$$
d x_{t}=\beta\left(\mathcal{L}\left(x_{t}\right), x_{t}\right) d \mathbf{Z}_{t},
$$


where $\mathcal{L}\left(x_{t}\right)$ denotes the law of $x$, and $\beta$ is an appropriate function on the space of measures, it is reasonable to expect that $t \mapsto \beta\left(\mathcal{L}\left(x_{t}\right), x\right)$ has such a decomposition and that one could solve the equation as a fixed-point in an appropriate space of measures.

Following this logic, if we want to consider the equation with added Brownian motion (4) as a fixed-point, this would necessitate being able to solve equation (5). The usual way, see [12], [13] and [14], to study this hybrid rough path and Itô equation is to consider the joint rough path

$$
\left(\begin{array}{c}
\mathbf{W}_{s t} \\
\mathbf{Z}_{s t}
\end{array}\right):=\left(\left(\begin{array}{c}
W_{t}-W_{s} \\
Z_{t}-Z_{s}
\end{array}\right), \quad\left(\begin{array}{cc}
\int_{s}^{t}\left(W_{r}-W_{s}\right) d W_{r} & \int_{s}^{t}\left(W_{r}-W_{s}\right) d Z_{r} \\
\int_{s}^{t}\left(Z_{r}-Z_{s}\right) d W_{r} & Z_{s t}
\end{array}\right)\right),
$$

and recast the equation on the form of a rough path equation

$$
d x_{t}=\left(\begin{array}{c}
\sigma_{t} \\
\beta_{t}
\end{array}\right)\left(x_{t}\right) d\left(\begin{array}{c}
\mathbf{W}_{t} \\
\mathbf{Z}_{t}
\end{array}\right) \text {. }
$$

Again, one would need to make an expansion of $\left(\sigma_{t}, \beta_{t}\right)^{T}$ in terms of the path $(W, Z)^{T}$. However, thinking towards the goal of solving mean-field equations, the simplest examples shows that there is no reason to expect that $\sigma_{t}$ is controlled by a fixed Brownian path in any sense - the law of the solution is an average over all Brownian sample paths.

Instead, if we define $W_{s t}^{\sigma}(x)=\int_{s}^{t} \sigma_{r}(x) d W_{r}$ as a Wiener-Itô integral and $Z_{s t}^{\beta}(x)=\int_{s}^{t} \beta_{r}(x) d \mathbf{Z}_{r}$ as a rough path integral, then on small time scales one would expect

$$
\left|\int_{s}^{t} \sigma_{r}\left(x_{r}\right) d W_{r}-W_{s t}^{\sigma}\left(x_{s}\right)\right| \vee\left|\int_{s}^{t} \beta_{r}\left(x_{r}\right) d \mathbf{Z}_{r}-Z_{s t}^{\beta}\left(x_{s}\right)\right|,
$$

to be small, so that one could use $W^{\sigma}$ and $Z^{\beta}$ to define a notion of non-linear 2 integration. At the heart of all stochastic integration is the difficulty that the above is not enough to guarantee a canonically defined integration map in the pathwise sense. The most fundamental understanding of the rough path theory is that one can construct integrals once additional information about the driving path is given by some off-line argument e.g. stochastic integration.

\section{Existing literature}

The stochastic equation, i.e. (11) and (2) has been studied in [20] and [21] but focusing on the case where the initial condition has a density. The measure-valued case was studied very recently in [8]. Under more restrictive conditions, either on the class of solutions or on the coefficients (like strong parabolicity), the well-posedness of solutions to SPDE of the type (2) had been previously considered by Dawson, Vaillancourt in [10].

McKean-Vlasov equations from a rough path perspective has already been introduced in [7] and, more recently in [1], focusing on the Lagrangian description. In [1] the equation is driven by a general random rough path, which gives the additional difficulty of needing to keep track of the rough path as a $L^{p}(\Omega)$-valued path. The latter space is present to consider a probability measure as the law of a random variable and Lions' approach to calculus for the Wasserstein metric. The approach by Gubinelli on controlled rough paths is then used to solve the equation as a fixed-point in the mixed $\mathbb{R}^{d}$ and $L^{p}(\Omega)$-space.

\footnotetext{
${ }^{2}$ We choose to call the integration non-linear since a mapping $x \mapsto \int f_{r}\left(x_{r}\right) d r$ is obviously never linear.
} 
We mention also [5] where the authors study mean-field games in the presence of a common noise as in (1). The authors use tightness arguments along with approximations to prove existence of a (probabilistically) weak solutions. Then, the authors prove a Yamada-Watanabe type principle for these equations to prove existence and uniqueness of (probabilistically) strong solutions.

In Section 3 we build a version of the rough path theory that allow for time dependent coefficients. The results in this section should be compared to [3] where the authors solves equations on this form. There, the main focus is flows build from a non-linear version of the sewing lemma. Very recently, right before the completion of the present paper, the authors of [23] introduce the very same object, here called a nonlinear rough path. The authors use a similar set up as in [17] to solve rough equations with time-dependent coefficients.

The papers [3] and [23] does not contain the same precise estimates as the present paper, which is crucially needed to set up a contraction mapping for the McKean-Vlasov equation (4).

\section{Main contributions}

The main contribution of this paper is the formulation and well-posedness of the nonlinear FokkerPlanck equation in terms of the appropriate rough path topology. We believe this is the first paper to study a rough non-local diffusion from both the Lagrangian and Eulerian perspective. Furthermore we believe it is the first work to prove well-posedness of an equation with a nonlinearity in the noise term on this form.

It is plausible that the well-posedness of the McKean-Vlasov equation equation in the present paper can be seen as a particular case of the equation studied in [1] by doing a rough path lift of $W$ and $\mathbf{Z}$ as in (6), but now as a rough path with values in an $L^{p}(\Omega)$-space. However, our proof of the well-posedness of the nonlinear Fokker-Planck equation necessitate well-posedness of a rough path equation with time-dependent coefficients. As already mentioned, it is not reasonable to expect that the coefficients could be controlled by a single Brownian path thus one could not use [1] for the time dependent case. Moreover, for the same reason, time dependent coefficients are also needed to understand the McKean-Vlasov equation as a fixed point of linear diffusions in an appropriate space of measures.

In addition, we prove a result on existence of a solution to a linear, possibly degenerate, rough PDE which could be of independent interest.

\section{Structure of the paper}

The paper is structured as follows. In Section 2 we introduce the necessary concepts from rough path theory, including controlled rough paths, that will be needed for the paper. In Section 3 we introduce the corresponding integration theory to handle non-linear integration and differential equations. In Section 4 we show how to concretely build rough drivers from Itô integration theory and the theory of controlled paths. These examples will also act exactly as the rough drivers needed to formulate the McKean-Vlasov equation as a fixed point. Moreover, this section contains an average, in $\Omega$, Itô formula that allows us to prove that the law of a diffusion solves the Fokker-Planck equation (linear or nonlinear). In Section 5 we prove well-posedness for a linear RPDE with time dependent coefficients. In Section 6 we construct the appropriate space for solving the McKean-Vlasov equation. In Section 7 we prove uniqueness of our main equation, which hinges on the results of the previous sections. 


\section{Notations and preliminary results}

\subsection{Hölder and p-variation spaces}

For $T>0$ we let $\Delta_{T}$ denote the simplex $\Delta_{T}=\left\{(s, t) \in[0, T]^{2}: s<t\right\}$. For $\zeta>0$ and a Banach space $E$ we denote by $C_{2}^{\zeta}([0, T] ; E)$ the space of all continuous mappings $g: \Delta_{T} \rightarrow E$ such that

$$
[g]_{\zeta, h ; E}:=\sup _{(s, t) \in \Delta_{T}:|t-s| \leq h} \frac{\left\|g_{s t}\right\|_{E}}{|t-s|^{\zeta}}<\infty .
$$

It can be checked that the above space is independent of $h$, and we will write for simplicity $[g]_{\alpha ; E}:=$ $[g]_{\alpha, T ; E}$. When it is clear from the context, we will also omit the Banach space $E$, writing $[g]_{\alpha, h}$ and $[g]_{\alpha}$. We let $C^{\zeta}([0, T] ; E)$ denote the space of all paths $f:[0, T] \rightarrow E$ such that the increment $\delta f_{s t}:=f_{t}-f_{s}$ belongs to $C_{2}^{\zeta}([0, T] ; E)$. For simplicity we will write $[f]_{\alpha, h ; E}:=[\delta f]_{\alpha, h ; E}$. It is well known that local and global Hölder norms are comparable for paths, in the sense that

$$
[f]_{\zeta ; E} \leq[f]_{\zeta, h ; E}\left(1 \vee 2 h^{\zeta-1}\right)
$$

for all $f \in C^{\zeta}([0, T] ; E)$ (see Exercise 4.25 in [15]). It is well known that the Hölder spaces are not separable. However, the subspace

$$
C_{0}^{\alpha}([0, T] ; E):=\left\{f \in C^{\alpha}([0, T] ; E): \lim _{h \rightarrow 0}[f]_{\alpha, h}=0\right\}
$$

is separable, as proved in Proposition A.4

We let $C_{2}^{p-\text { var }}([0, T] ; E)$ be the space of all continuous mappings $g: \Delta_{T} \rightarrow E$ such that

$$
\llbracket g \rrbracket_{p,[s, t] ; E}:=\left(\sup _{\pi} \sum_{\left\{t_{i}\right\}=\pi}\left\|g_{t_{i} t_{i+1}}\right\|_{E}^{p}\right)^{1 / p}<\infty
$$

where the above supremum is taken over all partitions $\pi$ of $[s, t]$. If we define $w_{g}(s, t):=\llbracket g \rrbracket_{p,[s, t] ; E}^{p}$ it can be shown that $(s, t) \mapsto w_{g}(s, t)$ is a control, namely continuous and superadditive i.e. $w_{g}(s, u)+$ $w_{g}(u, t) \leq w_{g}(s, t)$. Moreover, we see that if there exists a control $w$ such that $\left\|g_{s t}\right\|_{E} \leq w(s, t)^{1 / p}$, then $w_{g}(s, t) \leq w(s, t)$, so that we could equivalently define

$$
\llbracket g \rrbracket_{p,[s, t] ; E}=\inf \left\{w(s, t)^{1 / p} \mid w \text { is a control such that }\left\|g_{u v}\right\|_{E} \leq w(u, v)^{1 / p} \text { for } s \leq u<v \leq t\right\} .
$$

We will write $\llbracket g \rrbracket_{p ; E}:=\llbracket g \rrbracket_{p,[0, T] ; E}$ and when the space $E$ is clear from the context we will simply write $\llbracket g \rrbracket_{p,[s, t]}$ and $\llbracket g \rrbracket_{p}:=\llbracket g \rrbracket_{p,[0, T]}$.

To see the relationship between Hölder continuity and $p$-variation, notice that for any partition $\pi$ we have

$$
\sum_{\pi}\left\|g_{t_{i} t_{i+1}}\right\|_{E}^{p} \leq \sum_{\pi}[g]_{\alpha ; E}^{p}\left|t_{i+1}-t_{i}\right|^{\alpha p}=[g]_{\alpha ; E}^{p}|t-s|
$$

when $\alpha=1 / p$, which gives the bound

$$
w_{g}(s, t) \leq[g]_{\alpha ; E}^{1 / \alpha}|t-s| .
$$

Given a control $w$, we construct the greedy partition, following [15, Chapter 11]; for $\beta>0$, define the partition $\left\{\tau_{n}\right\}_{n}$ as

$$
\tau_{0}=s, \quad \tau_{n+1}=\inf \left\{t \mid w\left(\tau_{n}, t\right) \geq \beta, \tau_{n}<t \leq T\right\} \wedge T,
$$

so that $w\left(\tau_{n}, \tau_{n+1}\right)=\beta$, for all $n<N$, and $w\left(\tau_{N}, \tau_{N+1}\right) \leq \beta$. Define now the integer

$$
N_{\beta}(w,[s, t]):=\sup \left\{n \geq 0 \mid \tau_{n}<t\right\} .
$$




\subsection{Rough paths}

Assume $E$ is a Banach space and equip $E \otimes E$ with the projective tensor norm. We call a pair

$$
\mathbf{Z}:=(Z, \mathbb{Z}) \in C^{\alpha}([0, T] ; E) \times C_{2}^{2 \alpha}([0, T] ; E \otimes E)
$$

for $\alpha \in\left(\frac{1}{3}, \frac{1}{2}\right)$ a rough path provided Chen's relation,

$$
\delta \mathbb{Z}_{s \theta t}=Z_{s \theta} \otimes Z_{\theta t},
$$

holds where we have defined the second order increment operator $\delta g_{s \theta t}:=g_{s t}-g_{\theta t}-g_{s \theta}$. We denote by $\mathscr{C}^{\alpha}([0, T] ; E)$ the (non-linear) set of all rough paths which we equip with the subset metric,

$$
[\mathbf{Z}-\mathbf{X}]_{\alpha, h}:=[Z-X]_{\alpha, h}+[\mathbb{Z}-\mathbb{X}]_{2 \alpha, h} .
$$

For a path of bounded variation, $Z:[0, T] \rightarrow E$ there is a canonical rough path, $\mathbf{Z}=\left(Z, \int Z \otimes d Z\right)$ where the latter is the iterated integral $\left(\int Z \otimes d Z\right)_{s t}=\int_{s}^{t} Z_{s r} \otimes d Z_{r}$ which is well defined when $Z$ is of bounded variation. We denote by $\mathscr{C}_{g}^{\alpha}([0, T] ; E)$ the set of geometric rough paths, which is the closure of the set of bounded variation paths in the rough path metric.

We notice that if $\mathbf{Z}$ is geometric, then $\mathbf{Z}$ is also weakly geometric which means $\operatorname{sym}\left(\mathbb{Z}_{s t}\right)=\frac{1}{2} Z_{s t} \otimes$ $Z_{s t}$, and we denote by $\mathscr{C}_{w g}^{\alpha}([0, T] ; E)$ the set of all such rough paths. When $E$ is finite dimensional it is known that (see e.g. [16, Proposition 8.12]) if $\mathbf{Z}$ is weakly geometric, there exists a sequence of smooth paths $Z^{n}$ such that $\mathbf{Z}^{n} \rightarrow \mathbf{Z}$ in $\mathscr{C}^{\bar{\alpha}}([0, T] ; E)$ for all $\bar{\alpha}<\alpha$.

\section{Controlled space}

Given a path $Z$ taking values in $\mathbb{R}^{m}$ we denote by $\mathscr{D}_{Z}^{2 \alpha}([0, T] ; E)$ the (linear) space of all controlled path, given by pairs $\left(Y, Y^{\prime}\right)$ of mappings

$$
Y:[0, T] \rightarrow \mathcal{L}\left(\mathbb{R}^{m} ; E\right), \quad Y^{\prime}:[0, T] \rightarrow \mathcal{L}\left(\mathbb{R}^{m \times m} ; E\right)
$$

such that

$$
Y_{s t}^{\sharp}:=\delta Y_{s t}-Y_{s}^{\prime} Z_{s t}, \quad \Longrightarrow \quad Y^{\sharp} \in C_{2}^{2 \alpha}\left([0, T] ; \mathcal{L}\left(\mathbb{R}^{m} ; E\right)\right) .
$$

We call $Y^{\prime}$ the Gubinelli derivative of $Y$. The above definition is sometimes better understood in coordinates $Y_{s t}^{\sharp, i}:=\delta Y_{s t}^{i}-Y_{s}^{i, k} Z_{s t}^{k}$ where we abuse notation and write $Y^{i, k}$ for the matrix representing the Gubinelli derivative. Above and for the remainder of the paper we shall use the convention of summation over repeated indices. We equip the space of all controlled paths with the norm

$$
\left\|\left(Y, Y^{\prime}\right)\right\|_{Z, \alpha, h ; E}:=\left|Y_{0}\right|+\left[Y^{\prime}\right]_{\alpha, h ; E}+\left[Y^{\sharp}\right]_{2 \alpha, h ; E} .
$$

\section{Sewing lemma and rough path integration}

We recall here the main result used to obtain estimates in the theory of rough paths, namely the sewing lemma.

Lemma 2.1. Suppose $g: \Delta_{T} \rightarrow E$ is such that

$$
[\delta g]_{\zeta, h ; E}:=\sup _{s<\theta<t:|t-s| \leq h} \frac{\left\|\delta g_{s \theta t}\right\|_{E}}{|t-s|^{\zeta}}<\infty
$$


for some $\zeta>1$ and $h>0$. Then there exists a unique pair $I(g):[0, T] \rightarrow E$ and $I(g)^{\natural}: \Delta_{T} \rightarrow E$ such that

$$
\delta I(g)_{s t}=g_{s t}+I(g)_{s t}^{\natural}
$$

with $\left[I(g)^{\natural}\right]_{\zeta ; E} \leq C[\delta g]_{\zeta, h ; E}$ for $C$ depending only on $\zeta$.

In fact, we have $I(g)_{s t}:=\lim _{|\pi| \rightarrow 0} \sum_{\pi} g_{t_{i} t_{i+1}}$ and we think of $I(g)$ as being an integral with local expansion $g$.

With this in hand we can define the rough path integral. Given a rough path $\mathbf{Z}$ and a controlled path $\left(Y, Y^{\prime}\right) \in \mathscr{D}_{Z}^{2 \alpha}([0, T] ; E)$, define the local expansion

$$
g_{s t}:=Y_{s} Z_{s t}+Y_{s}^{\prime} \mathbb{Z}_{s t}:=Y_{s}^{k} Z_{s t}^{k}+Y_{s}^{k, l} \mathbb{Z}_{s t}^{l, k}
$$

Using Chen's relation it is straightforward to check that $[\delta g]_{3 \alpha ; E}<\infty$ and we shall write $\int Y d \mathbf{Z}:=$ $I(g)$.

This construction also gives rise to a new rough path, namely

$$
X_{t}=\int_{0}^{t} Y_{r} d \mathbf{Z}_{r}, \quad \mathbb{X}_{s t}=\int_{s}^{t} X_{r} \otimes Y_{r} d \mathbf{Z}_{r}-X_{s} \otimes X_{s t}
$$

where the latter integral is defined by the local expansion

$$
X_{s} \otimes Y_{s}^{k} Z_{s t}^{k}+\left(Y_{s}^{l} \otimes Y_{s}^{k}+X_{s} \otimes Y_{s}^{k, l}\right) \mathbb{Z}_{s t}^{l, k} .
$$

One can then check that $\mathbf{X}:=(X, \mathbb{X}) \in \mathscr{C}^{\alpha}([0, T] ; E)$ and that this operation is continuous from $\mathscr{D}_{Z}^{2 \alpha}([0, T] ; E)$ to $\mathscr{C}^{\alpha}([0, T] ; E)$. Moreover, at least when $E$ is a separable Hilbert space, weak geometricity is preserved under rough path integration as spelled out in Lemma A.2.

We shall also use the sewing lemma to get a priori estimates by a slight (straightforward) generalization of the sewing lemma. Assume that $g$ is such that there exists controls $w$ and $w_{*}$ and a positive function $k$ such that

$$
\left|\delta g_{\text {sut }}\right| \leq w(s, t)^{\zeta}\left(1+k_{s}\right), \quad\left|g_{s t}\right| \leq w_{*}(s, t)^{\zeta}
$$

for some $\zeta>1$. Then there exists a universal constant $C$ such that

$$
\left|g_{s t}\right| \leq C w(s, t)^{\zeta}\left(1+\sup _{r \in[s, t]} k_{r}\right)
$$

\subsection{Taylor's formula}

For a path $y:[0, T] \rightarrow \mathbb{R}^{d}$ and a function $g: \mathbb{R}^{d} \rightarrow V$ (where $V$ is a finite-dimensional vector space) we use the notation

$$
[g]_{s t}^{k, y}:=\int_{0}^{1}(1-\theta)^{k-1} g\left(y_{s}+\theta \delta y_{s t}\right) d \theta .
$$

With this notation at hand the first and second order Taylor's formula reads

$$
\delta g(y)_{s t}=[\nabla g]_{s t}^{1, y} \delta y_{s t}, \quad[g]_{s t}^{1, y}-g\left(y_{s}\right)=[\nabla g]_{s t}^{2, y} \delta y_{s t}
$$

respectively. We obviously get $\left|[g]_{s t}^{k, y}\right| \lesssim\|g\|_{\infty}$. 


\subsection{Wasserstein metric}

We shall work with the Wasserstein metric on measures on Hölder spaces, but since separability of the underlying space is required for the Wasserstein metric to give a complete space, we shall use the subspaces $C_{0}^{\alpha}\left([0, T] ; \mathbb{R}^{d}\right)$. When the dimension is clear from the context we shall simply write $C_{0}^{\alpha}$. Given two probability measure $\mu, v \in \mathcal{P}\left(C_{0}^{\alpha}\right)$ say that $\pi \in \mathcal{P}\left(C_{0}^{\alpha} \times C_{0}^{\alpha}\right)$ is a coupling of $\mu$ and $\nu$ provided its first (respectively second) marginal is equal to $\mu$ (respectively $v$ ). We define the Wasserstein metric

$$
W_{\rho}(\mu, v):=\inf _{\pi}\left(\int_{C_{0}^{\alpha} \times C_{0}^{\alpha}}[\omega-\bar{\omega}]_{\alpha}^{\rho} d \pi(\omega, \bar{\omega})\right)^{1 / \rho}
$$

where the above infimum ranges over all couplings $\pi$ of the measures $\mu$ and $v$. Since $C_{0}^{\alpha}$ is separable we have that $\mathcal{P}_{\rho}\left(C_{0}^{\alpha}\right)$ is a complete space w.r.t. $W_{\rho}$.

We note that the $\rho$-th moment of a probability measure $\mu$ can be written $W_{\rho}\left(\mu, \delta_{0}\right)^{\rho}$ where $\delta_{0}$ is the Dirac-Delta centered in the path constantly equal to 0 .

\subsection{Spatial function spaces}

We fix $d \in \mathbb{N}$. For any multi-index $\beta=\left(\beta_{1}, \ldots, \beta_{d}\right)$, we set

$$
D^{\beta}=\left(\frac{\partial}{\partial x_{1}}\right)^{\beta_{1}}\left(\frac{\partial}{\partial x_{2}}\right)^{\beta_{2}} \cdots\left(\frac{\partial}{\partial x_{d}}\right)^{\beta_{d}}
$$

and $|\beta|=\beta_{1}+\cdots+\beta_{d}$. For $p>1$ and an integer $k \geq 0$, we let $W^{k, p}=W^{k, p}\left(\mathbb{R}^{d}\right)$ be the Sobolev space of real-valued functions on $\mathbb{R}^{d}$ with finite norm

$$
\|f\|_{W^{k, p}}:=\left(\sum_{|\beta| \leq k} \int_{\mathbb{R}^{d}}\left|D^{\beta} f(x)\right|^{p} d x\right)^{\frac{1}{p}}<\infty .
$$

Let $H^{k}:=W^{k, 2}\left(\mathbb{R}^{d} ; \mathbb{R}^{d}\right)$, be the Sobolev space of square integrable functions over $\mathbb{R}^{d}$, endowed with the norm $\|\cdot\|_{H^{k}}:=\|\cdot\|_{W^{k, 2}}$. For a Hilbert space $H$, we endow the space of linear functionals $\mathcal{L}\left(\mathbb{R}^{d} ; H\right)$ with the Hilbert-Schmidt norm

$$
\|A\|_{\mathcal{L}\left(\mathbb{R}^{d} ; H\right)}:=\left(\sum_{i=0}^{d}\left\|A e_{i}\right\|_{H}^{2}\right)^{\frac{1}{2}}, \quad A \in \mathcal{L}\left(\mathbb{R}^{d} ; H\right) .
$$

Moreover, we call $M_{T}^{2}(H)$ the space of $H$-valued, time-continuous, square integrable martingales endowed with the norm

$$
\|M\|_{M_{T}^{2}(H)}:=\sup _{t \in[0, T]}\left\|M_{t}\right\|_{L_{\omega}^{2}} .
$$

Let $k>\frac{d}{2}$. We denote by $C_{b}^{3} \otimes H^{k}$ the space of continuous functions $f: \mathbb{R}^{d} \times \mathbb{R}^{d} \rightarrow \mathbb{R}^{d}$ such that

(i) For all $x \in \mathbb{R}^{d}$, the function $y \mapsto f(x, y) \in H^{k}$.

(ii) For all $y \in \mathbb{R}^{d}$, the function $x \mapsto f(x, y) \in C_{b}^{3}$. 
(iii) We have

$$
\|f\|_{C_{b}^{3} \otimes H^{k}}:=\left(\sum_{0 \leq i \leq 3,|\beta| \leq k} \sup _{x \in \mathbb{R}^{d}} \int_{\mathbb{R}^{d}}\left|\nabla_{1}^{i} D_{2}^{\beta} f(x, y)\right|^{2} d y\right)^{\frac{1}{2}}<\infty, \quad f \in C_{b}^{3} \otimes H^{k} .
$$

We endow the space $C_{b}^{3} \otimes H^{k}$ with the induced norm $\|f\|_{C_{b}^{3} \otimes H^{k}}$. Above we have used the Frechet derivative in the first variable and the weak derivative in the second variable.

Contrary to $H^{l} \otimes H^{k}$, this space is well suited for the convolution $f(x, y)=\sigma(x-y)$ and we see that $f \in C_{b}^{3} \otimes H^{k}$ if $\sigma \in H^{3+k}$.

\section{Non linear integration}

In this section we build the theory of rough paths to accommodate for time-dependent coefficients. We aim to solve the equation

$$
\dot{x}_{t}=f_{t}\left(x_{t}\right), \quad x_{0}=\xi \in \mathbb{R}^{d}
$$

for given function $f$ which is a distribution in time but regular in space. We shall use the framework akin to the definition by Davie in [9]. To illustrate the set up, assume that $x$ is a smooth solution of (17). Integrating the equation and using Taylor's formula we obtain

$$
\begin{aligned}
\delta x_{s t}= & \int_{s}^{t} f_{r}\left(x_{r}\right) d r=\int_{s}^{t} f_{r}\left(x_{s}\right)+\nabla f_{r}\left(x_{s}\right)\left(\delta x_{s r}\right)+\left[\nabla^{2} f_{r}\right]_{s r}^{2, x}\left(\delta x_{s r} \otimes \delta x_{s r}\right) d r \\
= & \int_{s}^{t} f_{r}\left(x_{s}\right) d r+\int_{s}^{t}\left[\nabla^{2} f_{r}\right]_{s r}^{2, x}\left(\delta x_{s r} \otimes \delta x_{s r}\right) d r \\
& +\int_{s}^{t} \nabla f_{r}\left(x_{s}\right)\left(\int_{s}^{r} f_{u}\left(x_{s}\right) d u+\int_{s}^{r} \nabla f_{u}\left(x_{s}\right)\left(\delta x_{s u}\right) d u+\int_{s}^{r}\left[\nabla^{2} f_{u}\right]_{s u}^{2, x}\left(\delta x_{s u} \otimes \delta x_{s u}\right) d u\right) d r \\
= & F_{s t}\left(x_{s}\right)+\mathbb{F}_{s t}\left(x_{s}\right)+x_{s t}^{\natural} .
\end{aligned}
$$

Here we have defined the driver $\mathbf{F}:=(F, \mathbb{F})$ of the equation as follows

$$
F_{s t}(x):=\int_{s}^{t} f_{r}(x) d r \quad \mathbb{F}_{s t}(x):=\int_{s}^{t} \nabla f_{r}(x) F_{s r}(x) d r
$$

and the remainder as

$$
x_{s t}^{\natural}:=\int_{s}^{t} \int_{s}^{r} \nabla f_{r}\left(x_{s}\right) \nabla f_{u}\left(x_{s}\right)\left(\delta x_{s u}\right)+\left[\nabla^{2} f_{u}\right]_{s u}^{2, x}\left(\delta x_{s u} \otimes \delta x_{s u}\right) d u d r+\int_{s}^{t}\left[\nabla^{2} f_{r}\right]_{s r}^{2, x}\left(\delta x_{s r} \otimes \delta x_{s r}\right) d r .
$$

With the above notation, we rewrite equation (17) as

$$
d x_{t}=\mathbf{F}_{d t}\left(x_{t}\right) .
$$

As is usual in rough path theory, we shall now read the definition (18) in the opposite direction - we assume we are given a pair of functions $(F, \mathbb{F})$ satisfying some compatibility conditions (in Definition 3.1 below), and take this as a definition of the non-linearity $f$. We will then take $x^{\natural}$ to be implicitly defined and say that $x$ is a solution provided $x^{\natural}$ is of high time regularity. 
We can read (17) in integral form as $x_{t}=x_{0}+\int_{0}^{t} \mathbf{F}_{d r}\left(x_{r}\right)$ and can be regarded as a rough version of the semimartingale integration theory by Kunita in [19].

We shall use a similar definition as in [3], with a noticeable difference that we allow our driver to depend on two spatial points. Moreover, we will not only be dealing with weakly geometric drivers.

Definition 3.1. For $p \in[2,3)$, a pair of functions $\mathbf{F}=(F, \mathbb{F}) \in C^{p-v a r}\left([0, T] ; C_{b}^{3}\left(\mathbb{R}^{d} ; \mathbb{R}^{d}\right)\right) \times$ $C_{2}^{\frac{p}{2}-\mathrm{var}}\left([0, T] ; C_{b}^{2}\left(\mathbb{R}^{d} \times \mathbb{R}^{d} ; \mathbb{R}^{d}\right)\right)$ is called a $p$-rough driver provided Chen's relation,

$$
\delta \mathbb{F}_{s u t}(x, y)=F_{s u}(x) \otimes \nabla F_{u t}(y):=F_{s u}^{i}(x) \partial_{i} F_{u t}(y)
$$

holds. The set of all such pairs is equipped with the metrics

$$
\llbracket \mathbf{F}-\mathbf{G} \rrbracket_{p,[s, t]}:=\llbracket F-G \rrbracket_{p,[s, t] ; C_{b}^{3}}+\sqrt{\llbracket \mathbb{F}-\mathbb{G} \rrbracket_{\frac{p}{2},[s, t] ; C_{b}^{2}}}
$$

Most of the time we will work on the diagonal of the spatial points and write simply $\mathbb{F}_{s t}(x):=$ $\mathbb{F}_{s t}(x, x)$, and we shall also write $\nabla F_{u t}(x) F_{s u}(x)=F_{s u}(x) \otimes \nabla F_{u t}(x)$.

For $\alpha \in\left(\frac{1}{3}, \frac{1}{2}\right]$ a pair of functions $\mathbf{F}=(F, \mathbb{F}) \in C^{\alpha}\left([0, T] ; C_{b}^{3}\left(\mathbb{R}^{d} ; \mathbb{R}^{d}\right)\right) \times C_{2}^{2 \alpha}\left([0, T] ; C_{b}^{2}\left(\mathbb{R}^{d} \times\right.\right.$ $\left.\left.\mathbb{R}^{d} ; \mathbb{R}^{d}\right)\right)$ is called an $\alpha$-rough driver provided (21) holds. The set of all such pairs is equipped with the metric

$$
[\mathbf{F}-\mathbf{G}]_{\alpha, h}:=\|F-G\|_{\alpha, h ; C_{b}^{3}}+\sqrt{\|\mathbb{F}-\mathbb{G}\|_{2 \alpha, h ; C_{b}^{2}}}
$$

Remark 3.2. The reason for using both p-variation and $\alpha$-Hölder continuous drivers is that the construction using Kolmogorov continuity theorem (Lemma 4.3 below) gives us more easily bounds in the sense of Hölder continuity. However, to estimate the difference between two solutions we need exponential bounds, and it is well known that even when $W$ is a Brownian motion, the random variable $[W]_{\alpha}$ is not exponentially integrable. This problem is circumvented by using p-variation, more specifically using the local accumulation $N_{1}\left(\|W\|_{p-v a r ;[, \cdot,]},[0, T]\right)$, see Section 4.2 for the details.

From (8) it is clear that if $\mathbf{F}$ is an $\alpha$-rough driver, then it is also a p-rough driver with $p=\frac{1}{\alpha}$. When the notion is clear from the context, we shall simply say that $\mathbf{F}$ is a rough driver.

Example 3.3. Consider a rough path $\mathbf{X} \in \mathscr{C}^{\alpha}\left([0, T] ; C_{b}^{3}\left(\mathbb{R}^{d} ; \mathbb{R}^{d}\right)\right)$, where we identify $C_{b}^{3}\left(\mathbb{R}^{d} ; \mathbb{R}^{d}\right) \otimes$ $C_{b}^{3}\left(\mathbb{R}^{d} ; \mathbb{R}^{d}\right)$ with a subspace 3 of $C_{b}^{3}\left(\mathbb{R}^{d} \times \mathbb{R}^{d} ; \mathbb{R}^{d \times d}\right)$ so that Chen's relation reads

$$
\delta \mathbb{X}_{s u t}^{i, j}(x, y)=X_{s u}^{i}(x) X_{u t}^{j}(y) .
$$

Let now $F_{s t}(x)=X_{s t}(x)$ and $\mathbb{F}_{s t}(x, y)=\nabla_{y}^{\otimes}\left(\mathbb{X}_{s t}(x, y)\right)$ where $\nabla_{2}^{\otimes}: C_{b}^{3}\left(\mathbb{R}^{d} \times \mathbb{R}^{d} ; \mathbb{R}^{d \times d}\right) \rightarrow C_{b}^{3}\left(\mathbb{R}^{d} \times\right.$ $\left.\mathbb{R}^{d} ; \mathbb{R}^{d}\right)$ is the multiplication of vector fields, i.e. the linear extension of the mapping defined by

$$
\left(\nabla_{2}^{\otimes}(f \otimes g)(x, y)\right)^{j}=f^{i}(x) \partial_{i} g^{j}(y) .
$$

It is straightforward to check that this gives a rough driver, and we notice that the mapping $\mathbf{X} \mapsto \mathbf{F}$ is continuous.

With this at hand we can define the notion of a solution.

\footnotetext{
${ }^{3}$ Since we are on the unbounded domain $\mathbb{R}^{d}$, we don't know if one can identify these spaces, but the inclusion is enough for our purposes
} 
Definition 3.4. Let $\mathbf{F}$ be a rough driver as in Definition 3.1 and $\xi \in \mathbb{R}^{d}$. A path $x:[0, T] \rightarrow \mathbb{R}^{d}$ is called a solution to (20) provided $x^{\natural}$ defined by

$$
\delta x_{s t}=F_{s t}\left(x_{s}\right)+\mathbb{F}_{s t}\left(x_{s}\right)+x_{s t}^{\natural}, \quad x_{0}=\xi,
$$

is such that $x^{\natural} \in C_{2}^{\frac{p}{3}-v a r}\left([0, T] ; \mathbb{R}^{d}\right)$.

Remark 3.5. One drawback with this method compared to linear integration is the lack of "universality" in the Itô-Lyons map; recall that the stochastic equation

$$
d x_{t}=V\left(x_{t}\right) \circ d B_{t}
$$

and its corresponding mapping $B \mapsto x$ can be factorized into a discontinuous map, $B \mapsto\left(B, \int B d B\right)$ and a continuous one $\left(B, \int B d B\right) \mapsto x$. One of the nice features of this decomposition is the fact that $B \mapsto\left(B, \int B d B\right)$ is universal in the sense that it does not depend on the vector field $V$ driving the equation, which allows to fix a subset $\Omega_{0} \subset \Omega$ for which one can do deterministic analysis on the differential equation.

In our case, however, the subset of $\Omega$ will depend on the driving vector fields since we are building a non-linear integration theory depending on the coefficients.

\subsection{A priori estimates}

Let $\mathbf{F}$ be a $p$-rough driver and assume $x$ is a solution of equation (20) in the sense of Definition 3.4. In this section we use (12) and (13) to deduce a priori estimates. We let $w_{\mathbf{F}}$ be the smallest control such that

$$
\left\|F_{s t}\right\|_{C_{b}^{3}} \leq w_{\mathbf{F}}(s, t)^{1 / p}, \quad\left\|\mathbb{F}_{s t}\right\|_{C_{b}^{2}} \leq w_{\mathbf{F}}(s, t)^{2 / p}
$$

Define the controlled quantity,

$$
x_{s t}^{\sharp}:=\delta x_{s t}-F_{s t}\left(x_{s}\right)=\mathbb{F}_{s t}\left(x_{s}\right)+x_{s t}^{\natural} .
$$

Lemma 3.6. Let $g \in C_{b}^{2}$, we have the following chain rule, $\forall s, t \in[0, T]$,

$$
g(x)_{s t}^{\sharp}:=\delta g(x)_{s t}-\nabla g\left(x_{s}\right) F_{s t}\left(x_{s}\right) \Longrightarrow\left|g(x)_{s t}^{\sharp}\right| \leq\|g\|_{C_{b}^{2}}\left(w_{\mathbf{F}}(s, t)^{1 / p} w_{x}(s, t)^{1 / p}+w_{x^{\sharp}}(s, t)^{2 / p}\right) .
$$

Proof. We have from Taylor's formula

$$
\delta g(x)_{s t}=[\nabla g]_{s t}^{1, x} \delta x_{s t}=[\nabla g]_{s t}^{1, x} F_{s t}\left(x_{s}\right)+[\nabla g]_{s t}^{1, x} x_{s t}^{\sharp}=\nabla g\left(x_{s}\right) F_{s t}\left(x_{s}\right)+g(x)_{s t}^{\sharp},
$$

where

$$
g(x)_{s t}^{\#}=\left([\nabla g]_{s t}^{1, x}-\nabla g\left(x_{s}\right)\right) F_{s t}\left(x_{s}\right)+[\nabla g]_{s t}^{1, x} x_{s t}^{\#} .
$$

By the definition of brackets (14), we get

$$
\left|[\nabla g]_{s t}^{1, x}-\nabla g\left(x_{s}\right)\right|=\left|\int_{0}^{1} \nabla g\left(x_{s}+\theta \delta x_{s t}\right)-\nabla g\left(x_{s}\right) d \theta\right| \leq\|g\|_{C_{b}^{2}}\left|\delta x_{s t}\right| .
$$

The result follows. 
With this in hand we turn to an a priori estimate for the nonlinear RDE.

Proposition 3.7. Let $0<h \leq T$. There exists constants $C$ and $h$ depending only on $p$ such that for all $s, t$ such that $w_{\mathbf{F}}(s, t) \leq h$ we have

$$
\left|x_{s t}\right| \leq C w_{\mathbf{F}}(s, t)^{1 / p}, \quad\left|x_{s t}^{\sharp}\right| \leq C w_{\mathbf{F}}(s, t)^{2 / p}, \quad\left|x_{s t}^{\natural}\right| \leq C w_{\mathbf{F}}(s, t)^{3 / p},
$$

Proof. We start with the easily verifiable identity for a function $G$ and path $y$

$$
\delta G(y)_{s u t}=\delta G_{s u t}\left(y_{s}\right)-\delta\left(G_{u t}(y .)\right)_{s u} .
$$

Using Chen's relation we get

$$
\begin{aligned}
\delta x_{s u t}^{\natural} & =\delta\left(F_{u t}(x .)\right)_{s u}+\delta\left(\mathbb{F}_{u t}(x .)\right)_{s u}-\delta \mathbb{F}_{s u t}\left(x_{s}\right) \\
& =\delta\left(F_{u t}(x .)\right)_{s u}-\nabla F_{u t}\left(x_{s}\right) F_{s u}\left(x_{s}\right)+\delta\left(\mathbb{F}_{u t}(x .)\right)_{s u} \\
& =F_{u t}(x)_{s u}^{\#}+\nabla \mathbb{F}_{u t}\left(x_{s}\right) F_{s u}\left(x_{s}\right)+\mathbb{F}_{u t}(x)_{s u}^{\sharp} .
\end{aligned}
$$

We get from Lemma 3.6, provided $h<1$

$$
\left|F_{u t}(x)_{s u}^{\sharp}\right|+\left|\mathbb{F}_{u t}(x)_{s u}^{\sharp}\right| \leq w_{\mathbf{F}}(s, t)^{1 / p}\left(w_{\mathbf{F}}(s, t)^{1 / p} w_{x}(s, t)^{1 / p}+w_{x^{\sharp}}(s, t)^{2 / p}\right)
$$

and clearly

$$
\left|\nabla \mathbb{F}_{u t}\left(x_{s}\right) F_{s u}\left(x_{s}\right)\right| \leq w_{\mathbf{F}}(s, t)^{3 / p} .
$$

From the sewing lemma there exists a constant $C$ such that

$$
\left|x_{s t}^{\natural}\right| \leq C\left(w_{\mathbf{F}}(s, t)^{2 / p} w_{x}(s, t)^{1 / p}+w_{\mathbf{F}}(s, t)^{1 / p} w_{x^{\sharp}}(s, t)^{2 / p}+w_{\mathbf{F}}(s, t)^{3 / p}\right)
$$

From equations (22) and (23) we have

$$
\left|x_{s t}\right| \leq w_{\mathbf{F}}(s, t)^{1 / p}+w_{\mathbf{F}}(s, t)^{2 / p}+w_{x^{\natural}}(s, t)^{3 / p} \quad\left|x_{s t}^{\sharp}\right| \leq w_{\mathbf{F}}(s, t)^{2 / p}+w_{x^{\natural}}(s, t)^{3 / p}
$$

and consequently

$$
\left|x_{s t}^{\natural}\right| \leq w_{x^{\natural}}(s, t)^{3 / p} \leq C\left(w_{\mathbf{F}}(s, t)^{1 / p} w_{x^{\natural}}(s, t)^{3 / p}+w_{\mathbf{F}}(s, t)^{3 / p}\right) .
$$

If now $s, t$ is such that $C w_{\mathbf{F}}(s, t)^{1 / p} \leq \frac{1}{2}$ we get

$$
w_{x^{\natural}}(s, t)^{3 / p} \leq C w_{\mathbf{F}}(s, t)^{3 / p}
$$

which gives

$$
\left|x_{s t}\right| \leq C w_{\mathbf{F}}(s, t)^{1 / p}, \quad\left|x_{s t}^{\sharp}\right| \leq C w_{\mathbf{F}}(s, t)^{2 / p} .
$$

The above bound translates now to global estimates on the solution itself in the following way.

Lemma 3.8. Assume now that $\mathbf{F}$ is an $\alpha$-rough driver with $\alpha=\frac{1}{p}$. Then we have, for $h>0$ small enough depending on $\mathbf{F}$,

$$
[x]_{\alpha, h} \leq C[\mathbf{F}]_{\alpha, h} .
$$

Moreover, we have the global estimate

$$
[x]_{\alpha} \leq C\left([\mathbf{F}]_{\alpha} \vee[\mathbf{F}]_{\alpha}^{1 / \alpha}\right)
$$

for a constant $C>0$ depending only on $\alpha$. 
Proof. Since $\mathbf{F}$ is Hölder continuous we have $w_{\mathbf{F}}(s, t) \leq[\mathbf{F}]_{\alpha, h}^{p}|t-s|$ for all $|t-s| \leq h$. Choose now $h$ such that $h^{\alpha}[\mathbf{F}]_{\alpha, h} C \leq \frac{1}{2}$ where $C$ is as in Proposition 3.7. For $|t-s| \leq h$ we have

$$
\left|x_{s t}\right| \leq C w_{\mathbf{F}}(s, t)^{\alpha} \leq C[\mathbf{F}]_{\alpha, h}|t-s|^{\alpha},
$$

from which (25) follows.

From (7) we get, choosing now $h \simeq[\mathbf{F}]_{\alpha}^{-1 / \alpha}, h^{\alpha-1} \simeq[\mathbf{F}]_{\alpha}^{(1-\alpha) / \alpha}$

$$
[x]_{\alpha} \leq[x]_{\alpha, h}\left(1 \vee 2 h^{\alpha-1}\right) \leq C[\mathbf{F}]_{\alpha}\left(1 \vee[\mathbf{F}]_{\alpha}^{(1-\alpha) / \alpha}\right)
$$

for some universal constant $C$ depending only on $\alpha$.

\subsection{A priori contractive estimates}

Let $p<3$, and assume $\mathbf{F}, \mathbf{G}$ are two $p$-rough drivers. We take two solutions $x$ and $y$ of equation (20) in the sense of Definition (3.4), with initial conditions $x_{0}$ and $y_{0}$ and driven by $\mathbf{F}$ and $\mathbf{G}$ respectively.

To illustrate the ideas of this section, we give the following remark.

Remark 3.9. Assume that $F:=\int_{0}^{t} f_{r}(x) d r, G:=\int_{0}^{t} g_{r}(x) d r, x$ and $y$ are smooth in time, so that we can write

$$
\begin{aligned}
& \left|x_{t}-y_{t}\right| \leq\left|x_{0}-y_{0}\right|+\left|\int_{0}^{t} f_{r}\left(x_{r}\right)-g_{r}\left(y_{r}\right) d r\right| \leq\left|x_{0}-y_{0}\right|+\int_{0}^{t}\left|f_{r}\left(x_{r}\right)-f_{r}\left(y_{r}\right)\right| d r+\int_{0}^{t}\left\|f_{r}-g_{r}\right\|_{C_{b}} d r \\
& \quad \leq\left|x_{0}-y_{0}\right|+\int_{0}^{t}\left\|\nabla f_{r}\right\|_{C_{b}}\left\|x_{r}-y_{r}\right\| d r+\int_{0}^{t}\left\|f_{r}-g_{r}\right\|_{C_{b}} d r \leq e^{\int_{0}^{t}\left\|\nabla f_{r}\right\|_{C_{b}} d r}\left(\left|x_{0}-y_{0}\right|+\int_{0}^{t}\left\|f_{r}-g_{r}\right\|_{C_{b}} d r\right)
\end{aligned}
$$

where we have used Gronwall's inequality in the last step. The purpose of this subsection is to replicate these estimates also for the rough case. The steps are similar to the previous subsection, except we compare two solutions.

We start by writing

$$
\delta x_{s t}-\delta y_{s t}=F_{s t}\left(x_{s}\right)-G_{s t}\left(y_{s}\right)+x_{s t}^{\sharp}-y_{s t}^{\sharp}=F_{s t}\left(x_{s}\right)-F_{s t}\left(y_{s}\right)+F_{s t}\left(y_{s}\right)-G_{s t}\left(y_{s}\right)+x_{s t}^{\sharp}-y_{s t}^{\sharp} .
$$

Let $z:=x-y$ and $z^{\sharp}:=x^{\sharp}-y^{\sharp}$ so that the above gives the estimate

$$
\left|\delta z_{s t}\right| \leq w_{\mathbf{F}}(s, t)^{1 / p}\left|z_{s}\right|+w_{\mathbf{F}-\mathbf{G}}(s, t)^{1 / p}+w_{z^{\sharp}}(t, s)^{1 / p} .
$$

We begin with the analogue of Lemma 3.6 that allows us to estimate nonlinearities of the remainders.

Lemma 3.10. Let $f, g \in C_{b}^{3}$. Then using the notation as in Lemma 3.6 we have the estimate

$$
\begin{aligned}
\left|f(x)_{s t}^{\sharp}-g(y)_{s t}^{\sharp}\right| & \leq\|f-g\|_{C_{b}^{2}} w_{\mathbf{F}}(s, t)^{2 / p}+\|g\|_{C_{b}^{3}}\left|z_{s}\right|\left(w_{\mathbf{F}}(s, t)^{2 / p}+w_{\mathbf{G}}(s, t)^{2 / p}\right) \\
& +\|g\|_{C_{b}^{2}}\left(w_{z}(s, t)^{1 / p} w_{\mathbf{F}}(s, t)^{1 / p}+w_{\mathbf{G}}(s, t)^{1 / p} w_{\mathbf{F}-\mathbf{G}}(s, t)^{1 / p}+w_{z^{\sharp}}(s, t)^{2 / p}\right) .
\end{aligned}
$$

Proof. We write

$$
f(x)_{s t}^{\sharp}-g(y)_{s t}^{\sharp}=\left[\nabla^{2} f\right]_{s t}^{2, x} \delta x_{s t} F_{s t}\left(x_{s}\right)-\left[\nabla^{2} g\right]_{s t}^{2, y} \delta y_{s t} G_{s t}\left(y_{s}\right)+[\nabla f]_{s t}^{1, x} x_{s t}^{\sharp}-[\nabla g]_{s t}^{1, y} y_{s t}^{\sharp} .
$$


The first two terms above can be written

$$
\begin{aligned}
{\left[\nabla^{2} f\right]_{s t}^{2, x} \delta x_{s t} F_{s t}\left(x_{s}\right) } & -\left[\nabla^{2} g\right]_{s t}^{2, y} \delta y_{s t} G_{s t}\left(y_{s}\right)=\left(\left[\nabla^{2} f\right]_{s t}^{2, x}-\left[\nabla^{2} g\right]_{s t}^{2, x}\right) \delta x_{s t} F_{s t}\left(x_{s}\right) \\
& +\left(\left[\nabla^{2} g\right]_{s t}^{2, x}-\left[\nabla^{2} g\right]_{s t}^{2, y}\right) \delta x_{s t} F_{s t}\left(x_{s}\right)+\left[\nabla^{2} g\right]_{s t}^{2, y}\left(\delta x_{s t}-\delta y_{s t}\right) F_{s t}\left(x_{s}\right) \\
& +\left[\nabla^{2} g\right]_{s t}^{2, y} \delta y_{s t}\left(F_{s t}\left(x_{s}\right)-G_{s t}\left(x_{s}\right)\right)+\left[\nabla^{2} g\right]_{s t}^{2, y} \delta y_{s t}\left(G_{s t}\left(x_{s}\right)-G_{s t}\left(y_{s}\right)\right) .
\end{aligned}
$$

Which gives the bound

$$
\begin{aligned}
& \left|\left[\nabla^{2} f\right]_{s t}^{2, x} \delta x_{s t} F_{s t}\left(x_{s}\right)-\left[\nabla^{2} g\right]_{s t}^{2, y} \delta y_{s t} G_{s t}\left(y_{s}\right)\right| \leq\left\|\nabla^{2} f-\nabla^{2} g\right\|_{C_{b}} w_{\mathbf{F}}(s, t)^{2 / p}+\left\|\nabla^{3} g\right\|_{C_{b}}\left|z_{s}\right| w_{\mathbf{F}}(s, t)^{2 / p}
\end{aligned}
$$

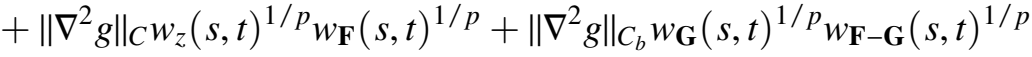

$$
\begin{aligned}
& +\left\|\nabla^{2} g\right\|_{C_{b}} w_{\mathbf{G}}(s, t)^{1 / p_{w_{\mathbf{G}}}}(s, t)^{1 / p}\left|z_{s}\right| \text {. }
\end{aligned}
$$

Now write

$$
[\nabla f]_{s t}^{1, x} x_{s t}^{\sharp}-[\nabla g]_{s t}^{1, y} y_{s t}^{\sharp}=\left([\nabla f]_{s t}^{1, x}-[\nabla g]_{s t}^{1, x}\right) x_{s t}^{\sharp}+\left([\nabla g]_{s t}^{1, x}-[\nabla g]_{s t}^{1, y}\right) x_{s t}^{\sharp}+[\nabla g]_{s t}^{1, y}\left(x_{s t}^{\#}-y_{s t}^{\sharp}\right) .
$$

We see that

$\left|\left([\nabla f]_{s t}^{1, x}-[\nabla g]_{s t}^{1, x}\right) x_{s t}^{\sharp}\right| \lesssim\|\nabla f-\nabla g\|_{C_{b}} w_{\mathbf{F}}(s, t)^{2 / p}, \quad$ and $\quad\left|\left([\nabla g]_{s t}^{1, y}\left(x_{s t}^{\sharp}-y_{s t}^{\sharp}\right)\right)\right| \lesssim\|\nabla g\|_{C_{b}} w_{z^{\sharp}}(s, t)^{2 / p}$ which gives (28).

Proposition 3.11. Assume that $\mathbf{F}$ and $\mathbf{G}$ are $\alpha$-rough drivers with $\alpha=\frac{1}{p}$. Then there exists universal constants $C$ such that

$$
\sup _{r \in[0, T]}\left|x_{r}-y_{r}\right| \leq C\left(\left|x_{0}-y_{0}\right|+\llbracket \mathbf{F}-\mathbf{G} \rrbracket_{p}\right) e^{C N\left(w_{\mathbf{F}},[0, T]\right)} .
$$

Moreover,

$$
\begin{aligned}
\llbracket x-y \rrbracket_{p,[s, t] \leq} \leq & e^{C N\left(w_{\mathbf{F}},[0, T]\right)}\left(\left|x_{0}-y_{0}\right|+\llbracket \mathbf{F}-\mathbf{G} \rrbracket_{p}\right) \\
& \cdot\left[\left(\llbracket \mathbf{F} \rrbracket_{p,[s, t]}+\llbracket \mathbf{G} \rrbracket_{p,[s, t]}\right)\left(1+\llbracket \mathbf{F} \rrbracket_{p}+\llbracket \mathbf{G} \rrbracket_{p}\right)^{2}\right] \\
\llbracket x^{\sharp}-y^{\sharp} \rrbracket_{\frac{p}{2},[s, t]} \leq & C e^{C N\left(w_{\mathbf{F}},[0, T]\right)}\left(\left|x_{0}-y_{0}\right|+\llbracket \mathbf{F}-\mathbf{G} \rrbracket_{p}\right) \\
& \cdot\left[\left(\llbracket \mathbf{F} \rrbracket_{p,[s, t]}+\llbracket \mathbf{G} \rrbracket_{p,[s, t]}\right)\left(1+\llbracket \mathbf{F} \rrbracket_{p}+\llbracket \mathbf{G} \rrbracket_{p}\right)^{2}\right]
\end{aligned}
$$

for all $s, t$ such that $C\left(\llbracket \mathbf{F} \rrbracket_{p,[s, t]}+\llbracket \mathbf{G} \rrbracket_{p,[s, t]}\right) \leq 1$. In particular, we have uniqueness for equation (20) and the solution is continuous w.r.t. the initial condition.

Proof. Using Chen's relation we get

$$
\delta(x-y)_{s u t}^{\natural}=F_{u t}(x)_{s u}^{\sharp}-G_{u t}(y)_{s u}^{\sharp}+\nabla \mathbb{F}_{u t}\left(x_{s}\right) F_{s u}\left(x_{s}\right)-\nabla \mathbb{G}_{u t}\left(x_{s}\right) G_{s u}\left(y_{s}\right)+\mathbb{F}_{u t}(x)_{s u}^{\sharp}-\mathbb{G}_{u t}(x)_{s u}^{\sharp} .
$$

Replacing $f=F_{u t}$ and $g=G_{u t}$ in (28) we get

$$
\begin{aligned}
& \left|F_{u t}(x)_{s u}^{\sharp}-G_{u t}(y)_{s u}^{\sharp}\right| \lesssim w_{\mathbf{F}-\mathbf{G}}(s, t)^{1 / p_{w}} w_{\mathbf{F}}(s, t)^{2 / p}+w_{\mathbf{G}}(s, t)^{1 / p}\left|z_{s}\right|\left(w_{\mathbf{F}}(s, t)^{2 / p}+w_{\mathbf{G}}(s, t)^{2 / p}\right) \\
& +w_{\mathbf{G}}(s, t)^{1 / p}\left(w_{z}(s, t)^{1 / p} w_{\mathbf{F}}(s, t)^{1 / p}+w_{\mathbf{G}}(s, t)^{1 / p} w_{\mathbf{F}-\mathbf{G}}(s, t)^{1 / p}+w_{z^{\sharp}}(s, t)^{2 / p}\right) .
\end{aligned}
$$


Replacing $f=\mathbb{F}_{u t}$ and $g=\mathbb{G}_{u t}$ in (28) we get

$$
\begin{aligned}
\left|\mathbb{F}_{u t}(x)_{s u}^{\#}-\mathbb{G}_{u t}(y)_{s u}^{\#}\right| & \lesssim w_{\mathbf{F}-\mathbf{G}}(s, t)^{2 / p_{w}} w_{\mathbf{F}}(s, t)^{2 / p}+w_{\mathbf{G}}(s, t)^{2 / p_{p}}\left|z_{s}\right|\left(w_{\mathbf{F}}(s, t)^{2 / p}+w_{\mathbf{G}}(s, t)^{2 / p}\right) \\
& +w_{\mathbf{G}}(s, t)^{2 / p}\left(w_{z}(s, t)^{1 / p} w_{\mathbf{F}}(s, t)^{1 / p}+w_{\mathbf{G}}(s, t)^{1 / p} w_{\mathbf{F}-\mathbf{G}}(s, t)^{1 / p}+w_{z^{\sharp}}(s, t)^{2 / p}\right) .
\end{aligned}
$$

Use also the estimate

$$
\left|z_{s t}^{\#}\right| \leq\left|z_{s}\right| w_{\mathbf{F}}(s, t)^{2 / p}+w_{\mathbf{F}-\mathbf{G}}(s, t)^{2 / p}+w_{z^{\natural}}(s, t)^{3 / p} .
$$

Let now $s, t$ be such that $w_{\mathbf{G}}(s, t)^{1 / p}, w_{\mathbf{F}}(s, t)^{1 / p} \leq \frac{C}{2}$ which gives

$$
\begin{aligned}
& \left|\delta z_{\text {sut }}^{\natural}\right| \lesssim w_{\mathbf{F}-\mathbf{G}}(s, t)^{1 / p} w_{\mathbf{F}}(s, t)^{2 / p}+w_{\mathbf{F}-\mathbf{G}}(s, t)^{2 / p} w_{\mathbf{F}}(s, t)^{1 / p} \\
& +w_{\mathbf{G}}(s, t)^{1 / p}\left|z_{s}\right|\left(w_{\mathbf{F}}(s, t)^{2 / p}+w_{\mathbf{G}}(s, t)^{2 / p}\right)
\end{aligned}
$$

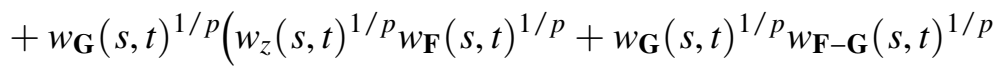

$$
\begin{aligned}
& +\left|z_{s}\right| w_{\mathbf{F}}(s, t)^{2 / p}+w_{\mathbf{F}-\mathbf{G}}(s, t)^{2 / p}+w_{z^{\natural}}(s, t)^{3 / p} .
\end{aligned}
$$

From (12) and (13) we get that there exists a universal constant $C$ such that

$$
\begin{aligned}
w_{z^{\natural}}(s, t)^{3 / p} \leq & C\left(w_{\mathbf{F}-\mathbf{G}}(s, t)^{1 / p} w_{\mathbf{F}}(s, t)^{2 / p}+w_{\mathbf{F}-\mathbf{G}}(s, t)^{2 / p} w_{\mathbf{F}}(s, t)^{1 / p}\right. \\
& +\sup _{r \in[s, t]}\left|z_{r}\right|\left(w_{\mathbf{G}}(s, t)^{1 / p_{1}} w_{\mathbf{F}}(s, t)^{2 / p}+w_{\mathbf{G}}(s, t)^{3 / p}\right) \\
& +w_{\mathbf{G}}(s, t)^{1 / p}\left(w_{z}(s, t)^{1 / p} w_{\mathbf{F}}(s, t)^{1 / p}+w_{\mathbf{G}}(s, t)^{1 / p} w_{\mathbf{F}-\mathbf{G}}(s, t)^{1 / p}\right. \\
& \left.+w_{\mathbf{F}-\mathbf{G}}(s, t)^{2 / p}+w_{z^{\natural}}(s, t)^{3 / p}\right) .
\end{aligned}
$$

Choose now $s, t$ such that $w_{\mathbf{G}}(s, t)^{1 / p} \leq \frac{C}{2} \wedge 1$, so that

$$
\begin{aligned}
w_{z^{\natural}}(s, t)^{3 / p} \leq & 2 C\left(w_{\mathbf{F}-\mathbf{G}}(s, t)^{1 / p} w_{\mathbf{F}}(s, t)^{2 / p}+w_{\mathbf{F}-\mathbf{G}}(s, t)^{2 / p} w_{\mathbf{F}}(s, t)^{1 / p}\right. \\
& +\sup _{r \in[s, t]}\left|z_{r}\right|\left(w_{\mathbf{G}}(s, t)^{1 / p} w_{\mathbf{F}}(s, t)^{2 / p}+w_{\mathbf{G}}(s, t)^{3 / p}\right) \\
& +w_{\mathbf{G}}(s, t)^{1 / p}\left(w_{z}(s, t)^{1 / p^{1 / p}} w_{\mathbf{F}}(s, t)^{1 / p}+w_{\mathbf{G}}(s, t)^{1 / p} w_{\mathbf{F}-\mathbf{G}}(s, t)^{1 / p}+w_{\mathbf{F}-\mathbf{G}}(s, t)^{2 / p}\right) .
\end{aligned}
$$

For the solution we have

$$
\begin{aligned}
\left|\delta z_{s t}\right| & \leq w_{\mathbf{F}}(s, t)^{1 / p}\left|z_{s}\right|+w_{\mathbf{F}}(s, t)^{2 / p}\left|z_{s}\right|+w_{z^{\natural}}(s, t)^{3 / p} \\
& \leq C\left(w_{\mathbf{F}}(s, t)^{1 / p} \sup _{r \in[s, t]}\left|z_{r}\right|+w_{\mathbf{F}-\mathbf{G}}(s, t)^{1 / p} w_{\mathbf{F}}(s, t)^{2 / p}\right. \\
& \left.+w_{\mathbf{F}-\mathbf{G}}(s, t)^{2 / p} w_{\mathbf{F}}(s, t)^{1 / p}+w_{\mathbf{G}}(s, t)^{1 / p} w_{z}(s, t)^{1 / p} w_{\mathbf{F}}(s, t)^{1 / p}\right) .
\end{aligned}
$$

Let now $(s, t)$ be such that $w_{\mathbf{F}}(s, t)^{1 / p}, w_{\mathbf{G}}(s, t)^{1 / p} \leq \frac{C}{2}$ we get

$$
\left|\delta z_{s t}\right| \leq \omega_{z}(s, t)^{1 / p} \leq C\left(w_{\mathbf{F}}(s, t)^{1 / p} \sup _{r \in[s, t]}\left|z_{r}\right|+w_{*}(s, t)^{3 / p}\right),
$$

where $w_{*}(s, t)=w_{\mathbf{F}-\mathbf{G}}(s, t)^{1 / 3} w_{\mathbf{F}}(s, t)^{2 / 3}+w_{\mathbf{F}-\mathbf{G}}(s, t)^{2 / 3} w_{\mathbf{F}}(s, t)^{1 / 3}$. From the rough Gronwall lemma, [11, Lemma 2.11], we get

$$
\sup _{r \in[s, t]}\left|z_{r}\right| \leq C \exp \left\{C w_{\mathbf{F}}(s, t)\right\}\left(\left|z_{s}\right|+w_{*}(s, t)^{3 / p}\right),
$$


and we notice that this holds for all subintervals $[s, t]$, i.e. no smallness assumption. Now, choose the finest partition $\tau_{k}$ of $[s, t]$ such that $w_{\mathbf{F}}\left(\tau_{k}, \tau_{k+1}\right)=1$. We have

$$
\sup _{r \in\left[s, \tau_{1}\right]}\left|z_{r}\right| \leq C\left(\left|z_{s}\right|+w_{*}\left(s, \tau_{1}\right)^{3 / p}\right) e^{C},
$$

and on $\left[\tau_{1}, \tau_{2}\right]$ we get

$$
\begin{aligned}
\sup _{r \in\left[\tau_{1}, \tau_{2}\right]}\left|z_{r}\right| & \leq C\left(\left|z_{\tau_{1}}\right|+w_{*}\left(\tau_{1}, \tau_{2}\right)^{3 / p}\right) e^{C} \leq C\left(C\left(\left|z_{s}\right|+w_{*}\left(s, \tau_{1}\right)^{3 / p}\right) e^{C}+w_{*}\left(\tau_{1}, \tau_{2}\right)^{3 / p}\right) e^{C} \\
& \leq C^{2}\left(\left|z_{s}\right|+w_{*}\left(s, \tau_{2}\right)^{3 / p}\right) e^{2 C}
\end{aligned}
$$

provided $C>1$. An easy induction shows that

$$
\sup _{r \in\left[\tau_{n}, \tau_{n+1}\right]}\left|z_{r}\right| \leq C^{n}\left(\left|z_{s}\right|+w_{*}\left(s, \tau_{n+1}\right)^{3 / p}\right) e^{n C} \leq\left(\left|z_{s}\right|+w_{*}\left(s, \tau_{n+1}\right)^{3 / p}\right) e^{n(C+\ln (C))} .
$$

By definition of the greedy partition (9) we get

$$
\sup _{r \in[s, t]}\left|z_{r}\right| \leq C\left(\left|z_{s}\right|+w_{*}(s, t)^{3 / p}\right) e^{\tilde{C} N\left(w_{\mathbf{F}},[s, t]\right)}
$$

Letting $s=0$ and using the bound $w_{*}(0, T) \leq w_{\mathbf{F}-\mathbf{G}}(0, T) \leq[\mathbf{F}-\mathbf{G}]_{\alpha}$ this shows (29). To see (30) we plug the above into (27) to get

$$
\begin{aligned}
\left|\delta z_{s t}\right| & \leq w_{\mathbf{F}}(s, t)^{1 / p}\left|z_{s}\right|+w_{\mathbf{F}-\mathbf{G}}(s, t)^{1 / p}+w_{z^{\natural}}(s, t)^{3 / p} \\
& \leq w_{\mathbf{F}}(s, t)^{1 / p}\left|z_{s}\right|+w_{\mathbf{F}-\mathbf{G}}(s, t)^{1 / p}+2 C\left(w_{\mathbf{F}-\mathbf{G}}(s, t)^{1 / p} w_{\mathbf{F}}(s, t)^{2 / p}+w_{\mathbf{F}-\mathbf{G}}(s, t)^{2 / p} w_{\mathbf{F}}(s, t)^{1 / p}\right. \\
& +\sup _{r \in[s, t]}\left|z_{r}\right|\left(w_{\mathbf{G}}(s, t)^{1 / p} w_{\mathbf{F}}(s, t)^{2 / p}+w_{\mathbf{G}}(s, t)^{3 / p}\right) \\
& +w_{\mathbf{G}}(s, t)^{1 / p}\left(w_{z}(s, t)^{1 / p} w_{\mathbf{F}}(s, t)^{1 / p}+w_{\mathbf{G}}(s, t)^{1 / p} w_{\mathbf{F}-\mathbf{G}}(s, t)^{1 / p}+w_{\mathbf{F}-\mathbf{G}}(s, t)^{2 / p}\right) \\
& \leq\left(w_{\mathbf{F}}(s, t)^{1 / p}+w_{\mathbf{G}}(s, t)^{1 / p}\right) \sup _{r \in[s, t]}\left|z_{r}\right|+w_{\mathbf{F}-\mathbf{G}}(s, t)^{1 / p}+w_{\mathbf{G}}(s, t)^{1 / p} w_{\mathbf{F}}(s, t)^{1 / p} w_{z}(s, t)^{1 / p} \\
\leq & \left(w_{\mathbf{F}}(s, t)^{1 / p}+w_{\mathbf{G}}(s, t)^{1 / p}\right) \sup _{r \in[s, t]}\left|z_{r}\right|+w_{\mathbf{F}-\mathbf{G}}(s, t)^{1 / p}
\end{aligned}
$$

using $w_{\mathbf{F}}(s, t)^{1 / p}, w_{\mathbf{G}}(s, t)^{1 / p} \leq 1 / 2$ in the last step. Using (33) gives

$$
\begin{aligned}
& \left|\delta z_{s t}\right| \leq\left(w_{\mathbf{F}}(s, t)^{1 / p}+w_{\mathbf{G}}(s, t)^{1 / p}\right) C w_{*}(0, t)^{3 / p} e^{\tilde{C} N\left(w_{\mathbf{F}},[0, t]\right)}+w_{\mathbf{F}-\mathbf{G}}(s, t)^{1 / p} \\
& \leq\left[\left(\left|z_{0}\right|+w_{\mathbf{F}-\mathbf{G}}(0, T)^{1 / p} w_{\mathbf{F}}(0, T)^{2 / p}+w_{\mathbf{F}-\mathbf{G}}(0, T)^{2 / p} w_{\mathbf{F}}(0, T)^{1 / p}\right)\left(w_{\mathbf{F}}(s, t)^{1 / p}+w_{\mathbf{G}}(s, t)^{1 / p}\right)\right. \\
& \left.+w_{\mathbf{F}-\mathbf{G}}(s, t)^{1 / p}\right] C e^{\tilde{C} N\left(w_{\mathbf{F}},[0, T]\right)} \text {. }
\end{aligned}
$$

This gives (30). The bound (31) is proved in a similar way.

Corollary 3.12. Assume that $\mathbf{F}$ and $\mathbf{G}$ are $\alpha$-rough drivers with $\alpha=\frac{1}{p}$. Then there exists a universal constant $C$ such that,

$$
\begin{aligned}
{[x-y]_{\alpha} \leq } & C e^{C N\left(w_{\mathbf{F}},[0, T]\right)}\left(\left|x_{0}-y_{0}\right|+[\mathbf{F}-\mathbf{G}]_{\alpha}\right)\left(1+[\mathbf{F}]_{\alpha}+[\mathbf{G}]_{\alpha}\right)^{2} \\
& \cdot\left(\left([\mathbf{F}]_{\alpha}+[\mathbf{G}]_{\alpha}\right) \vee\left([\mathbf{F}]_{\alpha}+[\mathbf{G}]_{\alpha}\right)^{\frac{1}{\alpha}}\right)
\end{aligned}
$$


Proof. Use bounds on the form $w_{\mathbf{F}}(s, t) \leq[\mathbf{F}]_{\alpha, h}^{p}|t-s|$ for all $|t-s| \leq h$ in inequality (34). This gives the Hölder estimate

$$
\begin{aligned}
{[z]_{\alpha, h} } & \left.\leq C e^{C N\left(w_{\mathbf{F}},[0, T]\right)}\left[\left([\mathbf{F}-\mathbf{G}]_{\alpha}[\mathbf{F}]_{\alpha}^{2}+[\mathbf{F}-\mathbf{G}]_{\alpha}^{2}[\mathbf{F}]_{\alpha}\right)\left([\mathbf{F}]_{\alpha, h}+[\mathbf{G}]_{\alpha, h}\right)+[\mathbf{F}-\mathbf{G}]_{\alpha, h}\right)\right] \\
& \leq C e^{C N\left(w_{\mathbf{F}},[0, T]\right)}\left(\left|z_{0}\right|+[\mathbf{F}-\mathbf{G}]_{\alpha}\right)\left[\left([\mathbf{F}]_{\alpha, h}+[\mathbf{G}]_{\alpha, h}\right)\left(1+[\mathbf{F}]_{\alpha}+[\mathbf{G}]_{\alpha}\right)^{2}\right]
\end{aligned}
$$

which holds when $h$ is such that $|t-s| \leq h$ we have $w_{\mathbf{F}}(s, t)^{1 / p}, w_{\mathbf{G}}(s, t)^{1 / p} \lesssim 1$, in particular when $h^{\alpha}\left([\mathbf{F}]_{\alpha, h}+[\mathbf{G}]_{\alpha, h}\right) \lesssim 1$.

Let $C$ be the constant given by Proposition 3.11 and set $h=C^{-\frac{1}{\alpha}}\left([\mathbf{F}]_{\alpha}+[\mathbf{G}]_{\alpha}\right)^{-\frac{1}{\alpha}}$. It follows by (7) and Proposition 3.11 that (the value of $C$ changes in the following lines, but it only depends on $\alpha$ )

$$
\begin{aligned}
{[x-y]_{\alpha} \leq } & {[x-y]_{\alpha, h}\left(1 \vee 2 h^{\alpha-1}\right) } \\
\leq & C e^{C N\left(w_{\mathbf{F}},[0, T]\right)}\left(\left|x_{0}-y_{0}\right|+[\mathbf{F}-\mathbf{G}]_{\alpha}\right)\left(1+[\mathbf{F}]_{\alpha}+[\mathbf{G}]_{\alpha}\right)^{2}\left([\mathbf{F}]_{\alpha, h}+[\mathbf{G}]_{\alpha, h}\right) \\
& \cdot\left(1 \vee\left([\mathbf{F}]_{\alpha}+[\mathbf{G}]_{\alpha}\right)^{-1+\frac{1}{\alpha}}\right) .
\end{aligned}
$$

This concludes the proof.

\subsection{Well-posedness of nonlinear RDEs}

Since uniqueness of equation (20) follows from Proposition 3.11, it is only left to prove existence of a solution. We do so by using a Picard iteration.

Theorem 3.13. Let $\mathbf{F}$ be a p-variation rough driver. There exists a unique solution $x$ of equation (20), in the sense of Definition 3.4 with initial condition $\xi \in \mathbb{R}^{d}$.

Proof. Uniqueness is given by Proposition 3.11. We study now existence. Define $x_{t}^{0}=\xi, x_{t}^{1}=F_{0 t}(\xi)$ and

$$
a_{s t}^{1}:=F_{s t}\left(x_{s}^{1}\right)+\mathbb{F}_{s t}\left(x_{s}^{0}, x_{s}^{1}\right),
$$

which gives

$$
\begin{aligned}
\delta a_{s u t}^{1} & =-\delta F_{u t}\left(x^{1}\right)_{s u}-\delta \mathbb{F}_{u t}\left(x^{1}, x^{0}\right)_{s u}+\delta \mathbb{F}_{s u t}\left(x_{s}^{0}, x_{s}^{1}\right) \\
& =-\left[\nabla F_{u t}\right]_{s u}^{1, x^{1}} \delta x_{s u}^{1}-\delta \mathbb{F}_{u t}\left(x^{1}, x^{0}\right)_{s u}+F_{s u}\left(x_{s}^{0}\right) \otimes \nabla F_{u t}\left(x_{s}^{1}\right) \\
& =-\left[\nabla^{2} F_{u t}\right]_{s u}^{2, x^{1}}\left(\delta x_{s u}^{1} \otimes F_{s u}\left(x_{s}^{0}\right)\right)-\delta \mathbb{F}_{u t}\left(x^{1}, x^{0}\right)_{s u} \\
& =-\left[\nabla^{2} F_{u t}\right]_{s u}^{2, x^{1}}\left(F_{s u}\left(x_{s}^{0}\right) \otimes F_{s u}\left(x_{s}^{0}\right)\right)-\delta \mathbb{F}_{u t}\left(x^{1}, x^{0}\right)_{s u} \\
& \leq w_{\mathbf{F}}(s, t)^{3 / p}+\left\|\nabla \mathbb{F}_{u t}\right\|_{C_{b}} w_{\mathbf{F}}(s, t)^{1 / p} \leq 2 w_{\mathbf{F}}(s, t)^{3 / p} .
\end{aligned}
$$

Consequently, there exists a pair $\left(x^{2}, x^{2, \natural}\right)$ such that

$$
\delta x_{s t}^{2}=F_{s t}\left(x_{s}^{1}\right)+\mathbb{F}_{s t}\left(x_{s}^{1}, x_{s}^{0}\right)+x_{s t}^{2, \downarrow}
$$

and we have $\left|x_{s t}^{2, \natural}\right| \leq C w_{\mathbf{F}}(s, t)^{3 / p}$ for some universal constant $C$.

We prove inductively that there exists universal constants $C$ and $h$ such that for $w_{\mathbf{F}}(s, t) \leq h$ we have $\left|x_{s t}^{n, \natural}\right| \leq C w_{\mathbf{F}}(s, t)^{3 / p}$ and $\left|\delta x_{s t}^{n}\right| \leq 2 w_{\mathbf{F}}(s, t)^{1 / p}+C w_{\mathbf{F}}(s, t)^{3 / p}$.

Given $x^{n-1}$ and $x^{n}$ we let

$$
a_{s t}^{n}:=F_{s t}\left(x_{s}^{n}\right)+\mathbb{F}_{s t}\left(x_{s}^{n}, x_{s}^{n-1}\right) .
$$


We then get

$$
\begin{aligned}
\delta a_{s u t}^{n} & =-\delta F_{u t}\left(x^{n}\right)_{s u}-\delta \mathbb{F}_{u t}\left(x^{n}, x^{n-1}\right)_{s u}+F_{s u}\left(x_{s}^{n-1}\right) \otimes \nabla F_{u t}\left(x_{s}^{n}\right) \\
& =-\left[\nabla F_{u t}\right]_{s u}^{1, x^{n}} \delta x_{s u}^{n}-\delta \mathbb{F}_{u t}\left(x^{n}, x^{n-1}\right)_{s u}+\nabla F_{u t}\left(x_{s}^{n}\right) F_{s u}\left(x_{s}^{n-1}\right) \\
& =-\left[\nabla F_{u t}\right]_{s u}^{1, x^{n}}\left(F_{s u}\left(x_{s}^{n-1}\right)\right)-\left[\nabla F_{u t}\right]_{s u}^{1, x^{n}} \mathbb{F}_{s u}\left(x_{s}^{n-1}, x_{s}^{n-2}\right)-\left[\nabla F_{u t}\right]_{s u}^{1, x^{n}} x_{s t}^{n, \mathfrak{t}} \mathbb{F}_{s u}\left(x_{s}^{n-1}, x_{s}^{n-2}\right) \\
& -\delta \mathbb{F}_{u t}\left(x^{n}, x^{n-1}\right)_{s u}+\nabla F_{u t}\left(x_{s}^{n}\right) F_{s u}\left(x_{s}^{n-1}\right) \\
& =-\left[\nabla F_{u t}\right]_{s u}^{2, x^{n}}\left(\delta x_{s u}^{n-1} \otimes F_{s u}\left(x_{s}^{n-1}\right)\right)-\left[\nabla F_{u t}\right]_{s u}^{1, x^{n}} \mathbb{F}_{s u}\left(x_{s}^{n-1}, x_{s}^{n-2}\right) \\
& -\left[\nabla F_{u t}\right]_{s u}^{1, x^{n}} x_{s t}^{n, \mathfrak{t}} \mathbb{F}_{s u}\left(x_{s}^{n-1}, x_{s}^{n-2}\right)-\delta \mathbb{F}_{u t}\left(x^{n}, x^{n-1}\right)_{s u} .
\end{aligned}
$$

which gives

$$
\begin{aligned}
\left|\delta a_{\text {sut }}^{n}\right| & \leq w_{\mathbf{F}}(s, t)^{2 / p}\left(2 w_{\mathbf{F}}(s, t)^{1 / p}+C w_{\mathbf{F}}(s, t)^{3 / p}\right)+C w_{\mathbf{F}}(s, t)^{5 / p}+w_{\mathbf{F}}(s, t)^{3 / p}+C w_{\mathbf{F}}(s, t)^{5 / p} \\
& +2 w_{\mathbf{F}}(s, t)^{2 / p}\left(2 w_{\mathbf{F}}(s, t)^{1 / p}+C w_{\mathbf{F}}(s, t)^{3 / p}\right) \\
& =7 w_{\mathbf{F}}(s, t)^{3 / p}+5 C w_{\mathbf{F}}(s, t)^{5 / p} \leq 8 w_{\mathbf{F}}(s, t)^{3 / p}
\end{aligned}
$$

provided $h$ is such that $5 C w_{\mathbf{F}}(s, t)^{2 / p} \leq 1$. This gives that there exists $x^{n+1}, x^{n+1, \natural}$ such that

$$
\delta x_{s t}^{n+1}=F_{s t}\left(x_{s}^{n}\right)+\mathbb{F}_{s t}\left(x_{s}^{n}, x_{s}^{n-1}\right)+x_{s t}^{n+1, \natural}, \quad\left|x_{s t}^{n+1, \natural}\right| \leq C_{p} 8 w_{\mathbf{F}}(s, t)^{3 / p}
$$

so $C \geq C_{p} 8$ will do. Provided $h$ is such that $w_{\mathbf{F}}(s, t)^{1 / p} \leq 1$ we also get

$$
\left|\delta x_{s t}^{n}\right| \leq w_{\mathbf{F}}(s, t)^{1 / p}+w_{\mathbf{F}}(s, t)^{2 / p}+C w_{\mathbf{F}}(s, t)^{3 / p} \leq 2 w_{\mathbf{F}}(s, t)^{1 / p}+C w_{\mathbf{F}}(s, t)^{3 / p}
$$

which proves the induction hypothesis.

From Arzelà-Ascoli we get that there exists a subsequence $x^{n_{k}}$ converging in $C\left([0, T] ; \mathbb{R}^{d}\right)$ to some element $x$. Clearly we get

$$
\sup _{s, t}\left(\left|F_{s t}\left(x_{s}^{n_{k}}\right)-F_{s t}\left(x_{s}\right)\right| \vee\left|\mathbb{F}_{s t}\left(x_{s}^{n_{k}}\right)-\mathbb{F}_{s t}\left(x_{s}\right)\right|\right) \rightarrow 0
$$

Since all the terms of (35) (or rather, the one with $n$ replaced by $n_{k}$ ) converges, we get that also $x_{s t}^{n_{k}, \natural^{\prime}}$ must converge to a limit denoted $x_{s t}^{\natural}$. Then $x$ and $x^{\natural}$ satisfies (22) and from the uniform bounds on $x^{n_{k}, \natural}$ we see that $x$ indeed is a solution.

\section{Rough non-linearities}

In this section we show how to construct the rough drivers that are used for solving the McKeanVlasov equation (4). We start by constructing rough drivers corresponding to Itô theory, i.e. given a vector field $\sigma$ and a Brownian motion $W$, we want to define

$$
W_{s t}^{\sigma}(x)=\int_{s}^{t} \sigma_{r}(x) d W_{r}, \quad \mathbb{W}_{s t}^{\sigma}(x, y)=\int_{s}^{t} W_{s r}^{\sigma}(x) \nabla \sigma_{r}(y) d W_{r}
$$

where the latter integration is in the sense of Itô. As the following example demonstrates, it is not possible to simply integrate a function $\sigma \in C\left([0, T] ; C_{b}^{3}\left(\mathbb{R}^{d} ; \mathbb{R}^{d}\right)\right)$ to produce a rough driver. 
Example 4.1. Let $d=1$ and $\sigma_{r}(x)=\sin (r x)$, then the mapping $x \mapsto W_{s t}^{\sigma}(x)$ is $P$-a.s. unbounded as $x \rightarrow \infty$. Indeed, let $s=0$ and $t=1$ and $x=2 \pi n$ for $n \in \mathbb{N}$, then $\left\{W_{01}^{\sigma}(2 \pi n)\right\}_{n \in \mathbb{N}}$ is an i.i.d. Gaussian sequence, which P-a.s. diverges.

The above example shows that we need some decay on our vector fields as $|x| \rightarrow \infty$. We choose to assume that $\sigma$ belongs to a Sobolev space $H^{k}\left(\mathbb{R}^{d} ; \mathbb{R}^{d}\right)$ where $k$ is large enough to use Sobolev embedding to show that $\mathbf{W}^{\sigma}$ is a rough driver. The reason for this choice is the relatively simple and well established theory of Itô integration that is available for Hilbert spaces. We conjecture that this regularity can be significantly lowered (e.g. with decay as in [3, Corollary 9]) and leave this for future investigation.

Let $d, m \in \mathbb{N}$ be fixed and let $\mathbf{Z} \in \mathscr{C}_{g}^{\bar{\alpha}}\left([0, T], \mathbb{R}^{m}\right)$, for $\bar{\alpha} \in\left(\frac{1}{3}, \frac{1}{2}\right)$. In this section we assume the following

Assumptions 4.2. Let $k \in \mathbb{N} \cup\{0\}$, and $\alpha \in\left(\frac{1}{3}, \bar{\alpha}\right)$,

(i) Let $\left(\beta, \beta^{\prime}\right) \in \mathscr{D}_{Z}^{2 \alpha}\left([0, T] ; H^{k}\right)$, as in Section 2

(ii) Let $\sigma:[0, T] \rightarrow \mathcal{L}\left(\mathbb{R}^{d} ; H^{k}\right)$ be a continuous function, such that

$$
\|\sigma\|_{L_{t}^{\infty} \mathcal{L}\left(\mathbb{R}^{d} ; H^{k}\right)}=\sup _{t \in[0, T]}\left\|\sigma_{t}\right\|_{\mathcal{L}\left(\mathbb{R}^{d} ; H^{k}\right)}<\infty .
$$

(iii) Let $p=\alpha^{-1}$, then

$$
\llbracket \sigma \rrbracket_{p ; \mathcal{L}\left(\mathbb{R}^{d} ; H^{k}\right)}<+\infty
$$

To simplify the following discussion, we introduce the convenient notation

$$
L=L(\sigma, \beta, \mathbf{Z}):=\left(1+\|\sigma\|_{L_{t}^{\infty} \mathcal{L}\left(\mathbb{R}^{d} ; H^{k}\right)}+\left\|\left(\beta, \beta^{\prime}\right)\right\|_{Z, \alpha ; H^{k}}\right)\left(1+[\mathbf{Z}]_{\bar{\alpha}} \vee[\mathbf{Z}]_{\bar{\alpha}}^{\frac{1}{2}}\right)
$$

\subsection{Construction of the rough driver}

\subsubsection{Itô theory}

Let $\left(\Omega, \mathcal{F},\left(\mathcal{F}_{t}\right)_{t \in[0, T]}, P\right)$ be a filtered probability space and let $W$ be a $d$-dimensional Wiener process on it. We assume that $\sigma$ satisfies Assumption 4.2[(ii), for $k>3+\frac{d}{2}$. We define, for $0 \leq s \leq t \leq T$,

$$
W_{t}^{\sigma}:=\int_{0}^{t} \sigma_{r} d W_{r} \in M_{T}^{2}\left(H^{k}\right), \quad W_{s t}^{\sigma}:=W_{t}^{\sigma}-W_{s}^{\sigma},
$$

where the integral is defined in the sense of Itô on Hilbert spaces, see [22, Section 2]. Thanks to Burkholder-Davis-Gundy (BDG) inequality for Hilbert spaces, [22, Theorem 2.4.7], we have for all $\rho \geq 1$ and $0 \leq s \leq t \leq T$,

$$
E \sup _{r \in[s, t]}\left\|W_{s r}^{\sigma}\right\|_{H^{k}}^{\rho} \leq C_{\rho}\left(\int_{s}^{t}\left\|\sigma_{r}\right\|_{\mathcal{L}\left(\mathbb{R}^{d} ; H^{k}\right)}^{2} d r\right)^{\frac{\rho}{2}} \leq C_{\rho}\left\|\sigma_{t}\right\|_{L_{t}^{\infty} \mathcal{L}\left(\mathbb{R}^{d} ; H^{k}\right)}^{\rho}|t-s|^{\frac{\rho}{2}} .
$$

We consider now the time-continuous stochastic process,

$$
\left(W^{\sigma} \otimes \nabla\right) \sigma .:[0, T] \times \Omega \rightarrow \mathcal{L}\left(\mathbb{R}^{d} ; H^{k} \otimes H^{k-1}\right),
$$


with Hilbert-Schmidt norm (15) bounded as $\left\|\left(W_{t}^{\sigma} \otimes \nabla\right) \sigma_{t}\right\|_{\mathcal{L}\left(\mathbb{R}^{d} ; H^{k} \otimes H^{k-1}\right)} \leq\left\|W_{t}^{\sigma}\right\|_{H^{k}}\left\|\sigma_{t}\right\|_{\mathcal{L}\left(\mathbb{R}^{d} ; H^{k}\right)}$, for all $t \in[0, T]$. Using again Itô theory on Hilbert spaces, we have that $\int_{0}^{t}\left(W_{r}^{\sigma} \otimes \nabla\right) \sigma_{r} d W_{r} \in M_{T}^{2}\left(H^{k} \otimes\right.$ $\left.H^{k-1}\right)$ and we set, for $0 \leq s \leq t \leq T$,

$$
\mathbb{W}_{s t}^{\sigma}:=\int_{s}^{t}\left(W_{r}^{\sigma} \otimes \nabla\right) \sigma_{r} d W_{r}-\left(W_{s}^{\sigma} \otimes \nabla\right) W_{s t}^{\sigma}: \Omega \rightarrow H^{k} \otimes H^{k-1} .
$$

Applying again BDG inequality and inequality (38), we have for all $\rho \geq 1$ and $0 \leq s \leq t \leq T$,

$$
E \sup _{r \in[s, t]}\left\|\mathbb{W}_{s r}^{\sigma}\right\|_{H^{k} \otimes H^{k-1}}^{p} \leq C_{\rho}\left(\int_{s}^{t}\left(E\left\|W_{s r}^{\sigma}\right\|_{H^{k}}^{\rho}\right)^{\frac{2}{\rho}}\left\|\sigma_{r}\right\|_{\mathcal{L}\left(\mathbb{R}^{d} ; H^{k}\right)}^{2} d r\right)^{\frac{p}{2}} \leq C_{\rho}\|\sigma\|_{L_{t}^{\infty} \mathcal{L}\left(\mathbb{R}^{d} ; H^{k}\right)}^{2 \rho}|t-s|^{\rho} .
$$

Lemma 4.3. Let $W$ be a d-dimensional Wiener process on the filtered probability space $\left(\Omega, \mathcal{F},\left(\mathcal{F}_{t}\right)_{t \in[0, T]}, P\right)$ and let $\sigma$ satisfy Assumption 4.2[(ii), with $k>3+\frac{d}{2}$. Let $W^{\sigma}$ and $\mathbb{W}^{\sigma}$ be defined as in (37) and (39), respectively. Then, for every $\alpha \in\left(\frac{1}{3}, \frac{1}{2}\right)$, for P-a.e. $\omega$

$$
\mathbf{W}^{\sigma}:=\left(W^{\sigma}, \mathbb{W}^{\sigma}\right) \in C^{\alpha}\left([0, T] ; C_{b}^{3}\left(\mathbb{R}^{d} ; \mathbb{R}^{d}\right)\right) \times C_{2}^{2 \alpha}\left([0, T] ; C_{b}^{2}\left(\mathbb{R}^{d} \times \mathbb{R}^{d} ; \mathbb{R}^{d}\right)\right),
$$

is a rough driver in the sense of Definition 3.1 and for all $\rho>\frac{2}{1-2 \alpha}$, we have

$$
\left\|\left[W^{\sigma}\right]_{\alpha ; C_{b}^{3}}\right\|_{L_{\omega}^{\rho}} \lesssim\|\sigma\|_{L_{t}^{\infty} \mathcal{L}\left(\mathbb{R}^{d} ; H^{k}\right)}, \quad\left\|\left[\mathbb{W}^{\sigma}\right]_{2 \alpha ; C_{b}^{2}\left(\mathbb{R}^{d} \times \mathbb{R}^{d}\right)}\right\|_{L_{\omega}^{\rho}} \lesssim\|\sigma\|_{L_{t}^{\infty} \mathcal{L}\left(\mathbb{R}^{d} ; H^{k}\right)}^{2} .
$$

Moreover, on small time-intervals $|t-s| \leq h \leq T$ we have, for $\bar{\alpha} \in\left(\alpha, \frac{1}{2}\right)$,

$$
\left[W^{\sigma}\right]_{\alpha, h, C_{b}^{3}} \leq h^{\bar{\alpha}-\alpha}\left[W^{\sigma}\right]_{\bar{\alpha}, C_{b}^{3}}, \quad\left[\mathbb{W}^{\sigma}\right]_{2 \alpha, h, C_{b}^{2}\left(\mathbb{R}^{d} \times \mathbb{R}^{d}\right)} \leq h^{\bar{\alpha}-\alpha}\left[\mathbb{W}^{\sigma}\right]_{2 \bar{\alpha}, C_{b}^{2}\left(\mathbb{R}^{d} \times \mathbb{R}^{d}\right)}, \quad P-\text { a.s. }
$$

Proof. We first study the space regularity of W. From the choice of $k$, Sobolev's embedding Theorem [4, Corollary 9.13] and inequalities (38) and (40), we have that

$$
\begin{gathered}
E \sup _{r \in[s, t]}\left\|W_{s r}^{\sigma}\right\|_{C_{b}^{3}}^{\rho} \leq E \sup _{r \in[s, t]}\left\|W_{s r}^{\sigma}\right\|_{H^{k}}^{\rho} \leq C_{\rho}\left\|\sigma_{t}\right\|_{L_{t}^{\infty} \mathcal{L}\left(\mathbb{R}^{d} ; H^{k}\right)}^{\rho}|t-s|^{\frac{\rho}{2}}, \\
E \sup _{r \in[s, t]}\left\|\mathbb{W}_{s r}^{\sigma}\right\|_{C_{b}^{3} \otimes C_{b}^{2}}^{\rho} \leq E \sup _{r \in[s, t]}\left\|\mathbb{W}_{s r}^{\sigma}\right\|_{H^{k} \otimes H^{k-1}}^{\rho} \leq C_{\rho}\left\|\sigma_{t}\right\|_{L_{t}^{\infty} \mathcal{L}\left(\mathbb{R}^{d} ; H^{k}\right)}^{2 \rho}|t-s|^{\rho} .
\end{gathered}
$$

By the Kolmogorov continuity theorem A.1, we obtain (41).

We check now that Chen's relation (21) holds $P$-a.s.. Indeed, we have the following,

$$
\delta \mathbb{W}_{s u t}^{\sigma}=\int_{u}^{t}\left(W_{s u}^{\sigma} \otimes \nabla\right) \sigma_{r} d W_{r}=\left(W_{s u}^{\sigma} \otimes \nabla\right) W_{u t}^{\sigma}, \quad P-a . s .
$$

To justify the last equality we call $\tilde{H}:=\mathcal{L}\left(\mathbb{R}^{d} ; H^{k} \otimes H^{k-1}\right)$ and we note that $\left(W_{s u}^{\sigma} \otimes \nabla\right): \Omega \rightarrow$ $L\left(H^{k}, \tilde{H}\right)$ is an $\mathcal{F}_{u}$-measurable random variable taking values in the space of linear operators between two Hilbert spaces. Thanks to the fact that the operator $\left(W_{s u}^{\sigma} \otimes \nabla\right)$ is measurable with respect to the left-most point of the integral, one can easily adapt [22, Lemma 2.4.1] to show that it commutes with the stochastic integral.

We shall also need contractive estimates w.r.t. the vector field.

Lemma 4.4. Let $\sigma$ and $\theta$ satisfy Assumption 4.2[ii) with $k>3+\frac{d}{2}$. Let $\mathbf{W}^{\sigma}$ and $\mathbf{W}^{\theta}$ be rough drivers as constructed in Lemma 4.3 w.r.t. the vector fields $\sigma$ and $\theta$. Then, for all $\bar{\alpha} \in\left[\alpha, \frac{1}{2}\right)$ and all $\rho>\frac{2}{1-2 \bar{\alpha}}$, there exists $K_{\rho} \in L^{\rho}(\Omega)$, such that for all $h \leq T$,

$$
\left[\mathbf{W}^{\sigma}-\mathbf{W}^{\theta}\right]_{\alpha, h ; C_{b}^{3}} \leq h^{\bar{\alpha}-\alpha} K_{\rho}\left(1+\|\sigma\|_{L_{t}^{\infty} \mathcal{L}\left(\mathbb{R}^{d} ; H^{k}\right)}+\|\theta\|_{L_{t}^{\infty} \mathcal{L}\left(\mathbb{R}^{d} ; H^{k}\right)}\right)\|\sigma-\theta\|_{L_{t}^{\infty} \mathcal{L}\left(\mathbb{R}^{d} ; H^{k}\right)}, \quad P-\text { a.s. }
$$

Proof. The proof follows as an application of Kolmogorov continuity theorem as in Lemma 4.3 . 


\subsubsection{Gubinelli integration}

Let $\bar{\alpha} \in\left(\frac{1}{3}, \frac{1}{2}\right), \mathbf{Z} \in \mathscr{C}_{g}^{\bar{\alpha}}\left([0, T], \mathbb{R}^{m}\right)$ and let $\beta$ satisfy Assumption 4.2](i), for $k \in \mathbb{N} \cup\{0\}$ and $\alpha \in$ $\left(\frac{1}{3}, \bar{\alpha}\right)$. Using Gubinelli's integration theory (see [15, Chapter 4]) we define, for each $0 \leq s \leq t \leq T$,

$$
Z_{s t}^{\beta}:=\int_{s}^{t} \beta_{r} d \mathbf{Z}_{r} \in H^{k}
$$

which satisfies (see [15, Theorem 4.10])

$$
\left\|Z_{s t}^{\beta}-\beta_{s}^{j} Z_{s t}^{j}-\beta_{s}^{j, i} \mathbb{Z}_{s t}^{i, j}\right\|_{H^{k}} \leq C\left([Z]_{\alpha}\left\|\beta^{\sharp}\right\|_{2 \alpha ; \mathcal{L}\left(\mathbb{R}^{m} ; H^{k}\right)}+[\mathbb{Z}]_{2 \alpha}\left\|\beta^{\prime}\right\|_{\alpha ; \mathcal{L}\left(\mathbb{R}^{m \times m} ; H^{k}\right)}\right)|t-s|^{3 \alpha} .
$$

and we have,

$$
\left\|Z_{s t}^{\beta}\right\|_{H^{k}} \leq C\left\|\left(\beta, \beta^{\prime}\right)\right\|_{Z, \alpha ; H^{k}}[\mathbf{Z}]_{\alpha}|t-s|^{\alpha}, \quad\left\|\left(Z^{\beta}\right)_{s t}^{\#}\right\|_{H^{k}} \leq C\left\|\left(\beta, \beta^{\prime}\right)\right\|_{Z, \alpha ; H^{k}}[\mathbf{Z}]_{\alpha}|t-s|^{2 \alpha}
$$

For $t \in[0, T]$, we define $Z_{t}^{\beta}:=Z_{0 t}^{\beta}$ and we consider $\left(Z_{t}^{\beta} \otimes \nabla\right) \beta_{t} \in \mathcal{L}\left(\mathbb{R}^{m} ; H^{k} \otimes H^{k-1}\right)$, with Gubinelli derivative

$$
\left(Z_{t}^{\beta} \otimes \nabla\right) \beta_{t}^{\prime}+\left(\beta_{t} \otimes \nabla\right) \beta_{t} \in \mathcal{L}\left(\mathbb{R}^{m \times m} ; H^{k} \otimes H^{k-1}\right) .
$$

Consequently we can define the integral $\int_{S}^{t}\left(Z_{r}^{\beta} \otimes \nabla\right) \beta_{r} d \mathbf{Z}_{r} \in H^{k} \otimes H^{k-1}$ via the local expansion

$$
\begin{aligned}
& \left\|\int_{s}^{t}\left(Z_{r}^{\beta} \otimes \nabla\right) \beta_{r} d \mathbf{Z}_{r}-\left(Z_{s}^{\beta} \otimes \nabla\right) \beta_{s}^{j} Z_{s t}^{j}-\left(\left(Z_{t}^{\beta} \otimes \nabla\right) \beta_{t}^{j, i}+\left(\beta_{t}^{j} \otimes \nabla\right) \beta_{t}^{i}\right) \mathbb{Z}_{s t}^{i, j}\right\|_{H^{k} \otimes H^{k-1}} \\
& \quad \leq C\left([Z]_{\alpha}\left[\left(\left(Z^{\beta} \otimes \nabla\right) \beta\right)^{\sharp}\right]_{2 \alpha ; \mathcal{L}\left(\mathbb{R}^{m} ; H^{k} \otimes H^{k-1}\right)}+[\mathbb{Z}]_{2 \alpha}\left[\left(Z_{t}^{\beta} \otimes \nabla\right) \beta_{t}^{\prime}+\left(\beta_{t} \otimes \nabla\right) \beta_{t}\right]_{\alpha ; \mathcal{L}\left(\mathbb{R}^{m \times m} ; H^{k} \otimes H^{k-1}\right)}\right)|t-s|^{3 \alpha} .
\end{aligned}
$$

Defining

$$
\mathbb{Z}_{s t}^{\beta}:=\int_{s}^{t}\left(Z_{r}^{\beta} \otimes \nabla\right) \beta_{r} d \mathbf{Z}_{r}-\left(Z_{s}^{\beta} \otimes \nabla\right) Z_{s t}^{\beta}
$$

we get

$$
\left\|\mathbb{Z}_{s t}^{\beta}\right\|_{H^{k} \otimes H^{k-1}} \leq C\left\|\left(\beta, \beta^{\prime}\right)\right\|_{Z, \alpha ; H^{k}}^{2}\left([\mathbf{Z}]_{\alpha}+[\mathbf{Z}]_{\alpha}^{2}\right)|t-s|^{2 \alpha} .
$$

We have the following lemmas of which we omit the proofs as they follow quite easily from the discussion above, standard computations on rough integrals, and Sobolev embedding Theorem [4, Corollary 9.13].

Lemma 4.5. Let $\bar{\alpha} \in\left(\frac{1}{3}, \frac{1}{2}\right)$ and $\mathbf{Z} \in \mathscr{C}_{g}^{\bar{\alpha}}\left([0, T], \mathbb{R}^{m}\right)$. Assume that $\beta$ satisfies Assumption 4.2](i)] with $k \geq 4+\frac{d}{2}$ and $\alpha \in\left(\frac{1}{3}, \bar{\alpha}\right)$. Let $Z^{\beta}$ and $\mathbb{Z}^{\beta}$ be defined as in (43) and (45), respectively. Then,

$$
\mathbf{Z}^{\beta}:=\left(Z^{\beta}, \mathbb{Z}^{\beta}\right) \in C^{\alpha}\left([0, T] ; C_{b}^{3}\left(\mathbb{R}^{d} ; \mathbb{R}^{d}\right)\right) \times C_{2}^{2 \alpha}\left([0, T] ; C_{b}^{2}\left(\mathbb{R}^{d} \times \mathbb{R}^{d} ; \mathbb{R}^{d}\right)\right),
$$

is a rough driver in the sense of Definition 3.1 and we have for time intervals of size $h \leq T$,

$$
\left[Z_{s t}^{\beta}\right]_{Z, \alpha, h ; C_{b}^{3}} \leq C\left\|\left(\beta, \beta^{\prime}\right)\right\|_{Z, \alpha ; H^{k}}[\mathbf{Z}]_{\alpha, h}, \quad\left[\mathbb{Z}_{s t}^{\beta}\right]_{2 \alpha, h ; C_{b}^{3} \otimes C_{b}^{2}} \leq C\left\|\left(\beta, \beta^{\prime}\right)\right\|_{Z, \alpha ; H^{k}}^{2}\left(1+[\mathbf{Z}]_{\alpha, h}\right)[\mathbf{Z}]_{\alpha, h} .
$$

Lemma 4.6. Let $\bar{\alpha} \in\left(\frac{1}{3}, \frac{1}{2}\right)$ and $\mathbf{Z} \in \mathscr{C}_{g}^{\bar{\alpha}}\left([0, T], \mathbb{R}^{m}\right)$. Assume that $\beta$ and $\gamma$ satisfy Assumption 4.2|(i)] with $k \geq 4+\frac{d}{2}$ and $\alpha \in\left(\frac{1}{3}, \bar{\alpha}\right)$. Let $\mathbf{Z}^{\beta}$ and $\mathbf{Z}^{\gamma}$ be rough drivers constructed as in Lemma 4.5. Then, on time intervals of size $h \leq T$,

$$
\left[\mathbf{Z}^{\beta}-\mathbf{Z}^{\gamma}\right]_{\alpha, h ; C_{b}^{3}} \leq C\left(1+\left\|\left(\beta, \beta^{\prime}\right)\right\|_{Z, \alpha ; H^{k}}+\left\|\left(\gamma, \gamma^{\prime}\right)\right\|_{Z, \alpha ; H^{k}}\right)\left\|\left(\beta, \beta^{\prime}\right)-\left(\gamma, \gamma^{\prime}\right)\right\|_{Z, \alpha ; H^{k}}\left([\mathbf{Z}]_{\alpha, h} \vee[\mathbf{Z}]_{\alpha, h}^{\frac{1}{2}}\right)
$$


Let us show that the above definition coincides with the usual definition of solutions of rough path equations.

Lemma 4.7. Suppose $x:[0, T] \rightarrow \mathbb{R}^{d}$ is a solution of $d x_{t}=\mathbf{Z}_{d t}^{\beta}\left(x_{t}\right)$ in the sense of Definition 3.4 Then $x$ also solves the classical rough path equation driven by $Z$ with coefficient $\beta$, i.e. $(x, \beta(x)) \in \mathscr{D}_{Z}^{2 \alpha}$ satisfies the following equation in the sense of Davie [9],

$$
x_{t}=\xi+\int_{0}^{t} \beta_{r}\left(x_{r}\right) d \mathbf{Z}_{r}
$$

where the $\beta(x)$ is also controlled by $Z$ with Gubinelli derivative $\beta^{\prime}(x)+\nabla \beta(x) \beta(x)$.

Proof. Assume $x$ is a solution to the non-linear equation and let us show that it also satisfies

$$
\delta x_{s t}=\beta_{s}^{j}\left(x_{s}\right) Z_{s t}^{j}+\left(\beta_{s}^{j, i}\left(x_{s}\right)+\nabla \beta_{s}^{j}\left(x_{s}\right) \beta_{s}^{i}\left(x_{s}\right)\right) \mathbb{Z}_{s t}^{i, j}+\bar{x}_{s t}^{\natural}
$$

for some remainder $\bar{x}^{\natural}$. By definition of $Z^{\beta}$ we have

$$
\left|Z_{s t}^{\beta}\left(x_{s}\right)-\beta_{s}^{j}\left(x_{s}\right) Z_{s t}^{j}-\beta_{s}^{j, i}\left(x_{s}\right) \mathbb{Z}_{s t}^{i, j}\right| \lesssim|t-s|^{3 \alpha} .
$$

Moreover

$$
\begin{aligned}
\mid \mathbb{Z}_{s t}^{\beta}\left(x_{s}\right)- & \nabla \beta_{s}^{j}\left(x_{s}\right) \beta_{s}^{i}\left(x_{s}\right) \mathbb{Z}_{s t}^{i, j}|\leq| Z_{s}^{\beta}\left(x_{s}\right) \nabla \beta_{s}\left(x_{s}\right)^{j} Z_{s t}^{j}+Z_{s}^{\beta}\left(x_{s}\right) \nabla \beta_{s}^{j, i}\left(x_{s}\right) \mathbb{Z}_{s t}^{i, j}-\nabla Z_{s t}^{\beta}\left(x_{s}\right) Z_{s}^{\beta}\left(x_{s}\right) \mid \\
& +\left|\int_{s}^{t} Z_{r}^{\beta}\left(x_{s}\right) \nabla \beta\left(x_{s}\right) d \mathbf{Z}_{r}-Z_{s}^{\beta}\left(x_{s}\right) \nabla \beta_{s}^{j}\left(x_{s}\right) Z_{s t}^{j}-\left(Z_{s}^{\beta}\left(x_{s}\right) \nabla \beta_{s}^{j, i}\left(x_{s}\right)+\nabla \beta_{s}^{j}\left(x_{s}\right) \beta_{s}^{i}\left(x_{s}\right)\right) \mathbb{Z}_{s t}^{i, j}\right| \\
& \lesssim|t-s|^{3 \alpha}
\end{aligned}
$$

by definition of $Z^{\beta}$ and $\int_{s}^{t} \nabla \beta\left(x_{s}\right) Z_{r}^{\beta}\left(x_{s}\right) d \mathbf{Z}_{r}$. This shows that $\left|x_{s t}^{\natural}-\bar{x}_{s t}^{\natural}\right| \lesssim|t-s|^{3 \alpha}$ which proves that the solutions coincide. Notice that the above bounds depend on $\left\|\left(\beta, \beta^{\prime}\right)\right\|_{\alpha, Z ; H^{k}}$ only.

\subsubsection{Mixed Itô and rough path integration}

Let $W$ be a $d$-dimensional Wiener process on the filtered probability space $\left(\Omega, \mathcal{F},\left(\mathcal{F}_{t}\right)_{t \in[0, T]}, P\right)$. Let $\bar{\alpha} \in\left(\frac{1}{3}, \frac{1}{2}\right), \mathbf{Z} \in \mathscr{C}_{g}^{\bar{\alpha}}\left([0, T], \mathbb{R}^{m}\right)$. Assume that $\sigma$ and $\beta$ satisfy Assumption 4.2[(ii)] and 4.2[(i)] respectively, for $k \in \mathbb{N} \cup\{0\}$ and $\alpha \in\left(\frac{1}{3}, \bar{\alpha}\right)$. Let $W^{\sigma}$ be defined as in (37) and $Z^{\beta}$ be defined as in (43). We define

$$
F_{s t}:=W_{s t}^{\sigma}+Z_{s t}^{\beta} \text {. }
$$

We remark that the first term on the right hand side of the above equation is random, whereas the second is deterministic. Define heuristically

$$
\mathbb{F}_{s t}:=\mathbb{W}_{s t}^{\sigma}+\mathbb{Z}_{s t}^{\beta}+\int_{s}^{t}\left(Z_{s r}^{\beta} \otimes \nabla\right) \sigma_{r} d W_{r}+\int_{s}^{t}\left(W_{s r}^{\sigma} \otimes \nabla\right) \beta_{r} d \mathbf{Z}_{r}
$$

The first two terms in the right hand side are defined as in (39) and (45) respectively, we need to make the last two rigorous. For the third term, using the Itô theory in Hilbert spaces as we did is Section 4.1.1, we see that the integral

$$
\int_{s}^{t}\left(Z_{r}^{\beta} \otimes \nabla\right) \sigma_{r} d W_{r} \in M_{T}^{2}\left(H^{k} \otimes H^{k-1}\right),
$$


is well-defined. Indeed, we have $\left(Z_{r}^{\beta} \otimes \nabla\right) \sigma_{r} \in \mathcal{L}\left(\mathbb{R}^{d} ; H^{k} \otimes H^{k-1}\right)$ for all $0 \leq r \leq T$. Hence, we can define

$$
\int_{s}^{t}\left(Z_{s r}^{\beta} \otimes \nabla\right) \sigma_{r} d W_{r}:=\int_{s}^{t}\left(Z_{r}^{\beta} \otimes \nabla\right) \sigma_{r} d W_{r}-\left(Z_{s}^{\beta} \otimes \nabla\right) W_{s t}^{\sigma}: \Omega \rightarrow H^{k} \otimes H^{k-1}
$$

Similarly, we have $\left(\sigma_{r} \otimes \nabla\right) Z_{r}^{\beta} \in \mathcal{L}\left(\mathbb{R}^{d} ; H^{k} \otimes H^{k-1}\right)$ and $\int_{s}^{t}\left(\sigma_{r} \otimes \nabla\right) Z_{r}^{\beta} d W_{r} \in M_{T}^{2}\left(H^{k} \otimes H^{k-1}\right)$. We define

$$
\int_{s}^{t}\left(W_{s r}^{\sigma} \otimes \nabla\right) \beta_{r} d \mathbf{Z}_{r}:=\left(W_{s t}^{\sigma} \otimes \nabla\right) Z_{t}^{\beta}-\int_{s}^{t}\left(\sigma_{r} \otimes \nabla\right) Z_{r}^{\beta} d W_{r}: \Omega \rightarrow H^{k} \otimes H^{k-1} .
$$

Lemma 4.8. Let $W$ be a d-dimensional Wiener process on the filtered probability space $\left(\Omega, \mathcal{F},\left(\mathcal{F}_{t}\right)_{t \in[0, T]}, P\right)$. Let $\bar{\alpha} \in\left(\frac{1}{3}, \frac{1}{2}\right)$ and $\mathbf{Z} \in \mathscr{C}_{g}^{\bar{\alpha}}\left([0, T], \mathbb{R}^{m}\right)$. Assume that $\sigma$ and $\beta$ satisfy Assumption $4.2[(i i)]$ and 4.2$\left.](i)\right]$ respectively, with $k \geq 4+\frac{d}{2}$ and $\alpha \in\left(\frac{1}{3}, \bar{\alpha}\right)$. Let $F$ and $\mathbb{F}$ be defined as in (47) and (48), respectively. Then, for P-a.e. $\omega$,

$$
\mathbf{F}:=(F, \mathbb{F}) \in C^{\alpha}\left([0, T] ; C_{b}^{3}\left(\mathbb{R}^{d} ; \mathbb{R}^{d}\right)\right) \times C_{2}^{2 \alpha}\left([0, T] ; C_{b}^{2}\left(\mathbb{R}^{d} \times \mathbb{R}^{d} ; \mathbb{R}^{d}\right)\right),
$$

is a rough driver in the sense of Definition 3.1 Moreover, on time intervals of size $h \leq T$ we have that, for all $\rho>\frac{2}{1-2 \bar{\alpha}}$, there exists $K_{\rho} \in L^{\rho}(\Omega)$, such that, $P$-a.s.,

$$
[\mathbf{F}]_{\alpha, h ; C_{b}^{3}} \leq\left(h \vee h^{\frac{1}{2}}\right)^{\bar{\alpha}-\alpha} K_{\rho} L(\sigma, \beta, \mathbf{Z}),
$$

where $L$ is defined in (36).

Proof. It is immediate to verify that the couple $(F, \mathbb{F})$ satisfies Chen's relation (21). We give now estimates on the first order term (47). As a consequence of the definition of $F$ and Lemma 4.5, we have, on an interval of size $h \leq T$,

$$
\left\|[F]_{\alpha, h ; C_{b}^{3}}\right\|_{L_{\omega}^{\rho}} \leq\left\|\left[W^{\sigma}\right]_{\alpha, h ; C_{b}^{3}}\right\|_{L_{\omega}^{\rho}}+C\left\|\left(\beta, \beta^{\prime}\right)\right\|_{Z, \alpha, h ; H^{k}}[\mathbf{Z}]_{\alpha, h} .
$$

We use now Lemma 4.3 to control the first term in the right hand side.

Now we study the regularity of $\mathbb{F}$. Using BDG inequality [22, Theorem 2.4.7] and inequality (44), we have for all $\rho \geq 1$ and $0 \leq s \leq t \leq T, t-s \leq h$,

$$
\begin{aligned}
\left\|\int_{s}^{t}\left(Z_{s r}^{\beta} \otimes \nabla\right) \sigma_{r} d W_{r}\right\|_{L_{\omega}^{\rho}\left(H^{k} \otimes H^{k-1}\right)} & \leq C_{\rho}\left(\int_{s}^{t}\left\|\left(Z_{s r}^{\beta} \otimes \nabla\right) \sigma_{r}\right\|_{\mathcal{L}\left(\mathbb{R}^{d} ; H^{k} \otimes H^{k-1}\right)}^{2} d r\right)^{\frac{1}{2}} \\
& \leq C_{\rho}\|\sigma\|_{L_{t}^{\infty} \mathcal{L}\left(\mathbb{R}^{d}, H^{k}\right)}\left[Z^{\beta}\right]_{\alpha ; H^{k}}|t-s|^{\alpha+\frac{1}{2}} \\
& \leq C_{\rho}\|\sigma\|_{L_{t}^{\infty} \mathcal{L}\left(\mathbb{R}^{d}, H^{k}\right)}\left\|\left(\beta, \beta^{\prime}\right)\right\|_{Z, \alpha ; H^{k}}[\mathbf{Z}]_{\alpha, h}|t-s|^{\alpha+\frac{1}{2}}
\end{aligned}
$$

By Kolmogorov continuity theorem A.1, we obtain that for every $\rho>\frac{2}{1-2 \alpha}$ there exists $K_{\rho} \in L^{\rho}(\Omega)$, such that

$$
\left[\int_{s}^{t}\left(Z_{s r}^{\beta} \otimes \nabla\right) \sigma_{r} d W_{r}\right]_{2 \alpha ; H^{k} \otimes H^{k-1}} \leq K_{\rho}\|\sigma\|_{L_{t}^{\infty} \mathcal{L}\left(\mathbb{R}^{d}, H^{k}\right)}\left\|\left(\beta, \beta^{\prime}\right)\right\|_{Z, \alpha ; H^{k}}[\mathbf{Z}]_{\alpha}, \quad P-\text { a.s. }
$$

Similar considerations lead to

$$
\left[\int_{s}^{t}\left(W_{s r}^{\sigma} \otimes \nabla\right) \beta_{r} d \mathbf{Z}_{r}\right]_{2 \alpha ; H^{k} \otimes H^{k-1}} \leq K_{\rho}\|\sigma\|_{L_{t}^{\infty} \mathcal{L}\left(\mathbb{R}^{d}, H^{k}\right)}\left\|\left(\beta, \beta^{\prime}\right)\right\|_{Z, \alpha ; H^{k}}[\mathbf{Z}]_{\alpha}, \quad P-\text { a.s. }
$$


Putting together the last inequalities, Lemma 4.3 and Lemma 4.5 yields

$\left[\mathbb{F}_{s t}\right]_{2 \alpha, h ; H^{k} \otimes H^{k-1}} \leq K_{\rho}\left\|\left(\beta, \beta^{\prime}\right)\right\|_{Z, \alpha ; H^{k}}[\mathbf{Z}]_{\alpha, h}\left(\left\|\left(\beta, \beta^{\prime}\right)\right\|_{Z, \alpha ; H^{k}}\left(1+[\mathbf{Z}]_{\alpha, h}\right)+\|\sigma\|_{L_{t}^{\infty} \mathcal{L}\left(\mathbb{R}^{d}, H^{k}\right)}\right)+\left[\mathbb{W}^{\sigma}\right]_{2 \alpha, h ; H^{k} \otimes H^{k-1}}$.

Inequality (49) follows immediately from the Sobolev embedding theorem [4, Corollary 9.13] .

Lemma 4.9. Let $W$ be a d-dimensional Wiener process on the filtered probability space $\left(\Omega, \mathcal{F},\left(\mathcal{F}_{t}\right)_{t \in[0, T]}, P\right)$. Let $\bar{\alpha} \in\left(\frac{1}{3}, \frac{1}{2}\right)$ and $\mathbf{Z} \in \mathscr{C}_{g}^{\bar{\alpha}}\left([0, T], \mathbb{R}^{m}\right)$. Assume that $\sigma, \theta$ satisfy Assumption 4.2](ii)] and that $\beta, \gamma$ satisfy Assumption 4.2](i) with $k \geq 4+\frac{d}{2}$ and $\alpha \in\left(\frac{1}{3}, \bar{\alpha}\right)$. Let $\mathbf{F}$ and $\mathbf{G}$ be nonlinear rough drivers constructed from $F_{s t}:=W_{s t}^{\sigma}+Z_{s t}^{\beta}$ and $G_{s t}:=W_{s t}^{\theta}+Z_{s t}^{\gamma}$ as in Lemma 4.8

Then, for all $\rho>\frac{2}{1-2 \bar{\alpha}}$, there exists $K_{\rho} \in L^{\rho}(\Omega)$, such that for any time interval of size $h \leq T$,

$$
[\mathbf{F}-\mathbf{G}]_{\alpha, h ; C_{b}^{3}} \leq\left(h \vee h^{\frac{1}{2}}\right)^{\bar{\alpha}-\alpha} K_{\rho} M\left(\|\sigma-\theta\|_{L_{t}^{\infty} H^{k}}+\left\|\left(\beta, \beta^{\prime}\right)-\left(\gamma, \gamma^{\prime}\right)\right\|_{Z, \alpha, h ; H^{k}}\right), \quad P-a . s .
$$

where we set $M:=L(\sigma, \beta, \mathbf{Z})+L(\theta, \gamma, \mathbf{Z})$ and $L$ is defined as in (36).

Proof. We already have contractive estimates from Lemmas 4.4 and 4.6 for the Itô and Gubinelli terms. We look now at the mixed integrals. For every $p \geq 1$, we have, for $|t-s| \leq h \leq T$,

$$
\begin{aligned}
\left\|\int_{s}^{t}\left(Z_{s r}^{\beta} \otimes \nabla\right) \sigma_{r} d W_{r}-\int_{s}^{t}\left(Z_{s r}^{\gamma} \otimes \nabla\right) \theta_{r} d W_{r}\right\|_{L_{\omega}^{p} H^{k} \otimes H^{k-1}} \\
\leq\left\|\int_{s}^{t}\left(Z_{s r}^{\beta-\gamma} \otimes \nabla\right) \sigma_{r} d W_{r}\right\|_{L_{\omega}^{p} H^{k} \otimes H^{k-1}}+\left\|\int_{s}^{t}\left(Z_{s r}^{\gamma} \otimes \nabla\right)\left(\sigma_{r}-\theta_{r}\right) d W_{r}\right\|_{L_{\omega}^{p} H^{k} \otimes H^{k-1}} \\
\leq C_{p}\left[\|\sigma\|_{L_{t}^{\infty} \mathcal{L}\left(\mathbb{R}^{d}, H^{k}\right)}\left\|\left(\beta, \beta^{\prime}\right)-\left(\gamma, \gamma^{\prime}\right)\right\|_{Z, \alpha, h ; H^{k}}+\|\sigma-\theta\|_{L_{t}^{\infty} \mathcal{L}\left(\mathbb{R}^{d}, H^{k}\right)}\left\|\left(\gamma, \gamma^{\prime}\right)\right\|_{Z, \alpha, h ; H^{k}}\right] \\
\cdot[\mathbf{Z}]_{\alpha, h}|t-s|^{\alpha+\frac{1}{2}} .
\end{aligned}
$$

The same estimates is true for the other mixed term. We can conclude by applying Kolmogorov continuity theorem.

\subsection{Integrability of the random rough driver}

In this section we are concerned with the study of exponential moments of the random rough driver. We will use the approach introduced by [6] and described in [15, Chapter 11].

Lemma 4.10. Let $\left(\Omega:=C\left([0, T] ; \mathbb{R}^{m}\right), \mathcal{B}(\Omega), P\right)$ be the canonical Wiener space with CameronMartin space $\mathcal{H} \subset \Omega$. We define on this space the canonical Wiener process as $W_{t}(\omega)=\omega(t)$. Let $\bar{\alpha} \in\left(\frac{1}{3}, \frac{1}{2}\right)$ and $\mathbf{Z} \in \mathscr{C}_{g}^{\bar{\alpha}}\left([0, T], \mathbb{R}^{m}\right)$. Assume that $\sigma$ and $\beta$ satisfy Assumption 4.2 with $k \geq 4+\frac{d}{2}$ and $\alpha \in\left(\frac{1}{3}, \bar{\alpha}\right)$, and let $\mathbf{F}$ be defined as in Lemma 4.8 Let $p:=\alpha^{-1} \in(2,3)$ and $q \geq 1$, such that $\frac{1}{p}+\frac{1}{q}>1$. Then, there exists $C:=C(p, q)>0$ and a null set $N \subset \Omega$, such that, $\forall \omega \in N^{c}$, $\forall[s, t] \subset[0, T]$ and $\forall h \in C^{q-v a r}$,

$$
\llbracket \mathbf{F} \rrbracket_{p,[s, t]}(\omega) \leq C \llbracket \sigma \rrbracket_{p ;[s, t]}\left(g_{s t}(\omega-h)+\llbracket h \rrbracket_{q ;[s, t]}\right),
$$

where, $g_{s, t}: \Omega \rightarrow \mathbb{R}_{+}$is defined as

$$
\begin{aligned}
g_{s, t}:= & \llbracket \mathbf{F} \rrbracket_{p,[s, t]}+\llbracket\left(W^{\sigma} \otimes \nabla\right) \sigma \rrbracket_{p ;[s, t]}+\llbracket(\sigma \otimes \nabla) W^{\sigma} \rrbracket_{p ;[s, t]} \\
& +\llbracket\left(Z^{\beta} \otimes \nabla\right) \sigma \rrbracket_{p ;[s, t]}+\llbracket(\sigma \otimes \nabla) Z^{\beta} \rrbracket_{p ;[s, t]}
\end{aligned}
$$


Proof. The proof of this result follows very closely the proof of [15, Theorem 11.5]. We repeat here the important pieces, where the dependence of the stochastic integrals on the space parameter $x$ has to be taken into account. We look at the first order term of $\mathbf{F}$. By definition, we have

$$
F_{s t}(\omega)=W_{s t}^{\sigma}(\omega)+Z_{s t}^{\beta}
$$

For every $s, t \in[0, T]$, the term $W_{s t}^{\sigma}$ is constructed as an $L_{\omega}^{2} H^{k}$ limit, hence there exists a sequence of partitions $\left(\Pi_{m}\right)_{m \in \mathbb{N}}$ and a null set $N_{s t}$ such that

$$
W_{s t}^{\sigma}(\omega)=\lim _{m \rightarrow \infty} \int_{\Pi_{m}} \sigma_{r} d W_{r}(\omega):=\lim _{m \rightarrow \infty} \sum_{t_{i} \in \Pi_{m}} \sigma_{t_{i}}\left(W_{t_{i+1}}(\omega)-W_{t_{i}}(\omega)\right),
$$

for every $\omega \in N_{s t}^{c}$. We call $N_{1}$ the intersection of $N_{s t}$ over all dyadic times and we note that it is still a null set. Similarly, we can construct a null set $N_{2}$ such that the function $W^{\sigma}(\omega)$ is of bounded $p$-variation for every $\omega \in N_{2}^{c}$. Let $\omega \in N_{1}^{c} \cap N_{2}^{c}$, we have,

$$
\lim _{m \rightarrow \infty} \int_{\Pi_{m}} \sigma_{r} d W_{r}(\omega+h)=\lim _{m \rightarrow \infty} \int_{\Pi_{m}} \sigma_{r}(x) d W_{r}(\omega)+\lim _{m \rightarrow \infty} \int_{\Pi_{m}} \sigma_{r}(x) d h_{r}
$$

The first limit on the right hand side exists because of the choice of the null set that we made in (52). The last limit is well defined as a Young integral, since $\sigma$ and $h$ are of complementary variation, see [15, Section 4.1]. Hence, also the left hand side of 53 converges and is, by definition, $W_{s t}^{\sigma}(\omega+h)$.

Hence, we obtain, $\forall \omega \in N_{1}^{c} \cap N_{2}^{c}, h \in C^{q-v a r}$, and for all dyadic times $[s, t] \subset[0, T]$,

$$
F_{s t}(\omega)=F_{s t}(\omega-h)+\int_{s}^{t} \sigma_{r} d h_{r}
$$

To generalize to any subset $[s, t] \subset[0, T]$, we can use a continuity argument, see [15, Theorem 11.5].

We compute now the $p$-variation in equation (54) and we obtain

$$
\llbracket F \rrbracket_{p,[s, t]}(\omega) \leq C_{p} \llbracket \sigma \rrbracket_{p,[s, t]}\left(\llbracket F \rrbracket_{p,[s, t]}(\omega-h)+\llbracket h \rrbracket_{q,[s, t]}\right) .
$$

Proceeding similarly for the second order term $\mathbb{F}$, we have that there exists a null set $N \subset \Omega$ such that $\forall \omega \in N^{c}, \forall h \in C^{q-v a r}$ and for all times $[s, t] \subset[0, T]$,

$$
\begin{aligned}
\mathbb{F}_{s t}(\omega)= & \mathbb{F}_{s t}(\omega-h)+\int_{s}^{t}\left(W_{s r}^{\sigma}(\omega-h) \otimes \nabla\right) \sigma_{r} d h_{r} \\
& +\int_{s}^{t}\left(\sigma_{u} \otimes \nabla\right) W_{u t}^{\sigma}(\omega-h) d h_{u}+\int_{s}^{t} \int_{s}^{r}\left(\sigma_{u} \otimes \nabla\right) \sigma_{r} d h_{u}^{l} d h_{r} \\
& +\left(\left(Z_{0}^{\beta} \otimes \nabla\right) \int_{0} \sigma_{r} d h_{r}\right)_{s t}-\int_{s}^{t}\left(Z_{s r}^{\beta} \otimes \nabla\right) \sigma_{r} d h_{r}+\int_{s}^{t}\left(\sigma_{r} \otimes \nabla\right) Z_{s r}^{\beta} d h_{r} .
\end{aligned}
$$

to obtain the third term on the right hand side, we used stochastic Fubini Theorem as follows

$$
\int_{s}^{t} \int_{s}^{r}\left(\sigma_{u} \otimes \nabla\right) \sigma_{r} d h_{u} d W_{r}(\omega-h)=\int_{s}^{t}\left(\sigma_{u} \otimes \nabla\right) W_{u t}^{\sigma}(\omega-h) d h_{u} .
$$

We compute the $p$-variation for the second order term. Using inequalities of the type $\sqrt{a b} \leq \sqrt{a}+\sqrt{b}$, for $a, b \in \mathbb{R}_{+}$, we obtain, for all $\omega \in N$,

$$
\llbracket \mathbb{F} \rrbracket_{p,[s, t]}^{\frac{1}{2}}(\omega) \leq C_{p} \llbracket \sigma \rrbracket_{p,[s, t]}\left(g(\omega-h)+\llbracket h \rrbracket_{q,[s, t]}\right),
$$

where $g_{s, t}$ is defined in (51). This concludes the proof 
For every $s, t \in[0, T]$, we define the control $w_{\mathbf{F}}(s, t)=\llbracket \mathbf{F} \rrbracket_{p,[s, t]}^{p}$ and we construct the greedy partition, following the construction in Section 2.1. Let $N_{\beta}$ be defined as in (9), for any $\beta>0$. We call $N$ the integer-valued random variable given by

$$
N(\omega):=N_{1}\left(w_{\mathbf{F}},[0, T]\right)(\omega),
$$

for $\omega \in \Omega$. For $y \geq 0$, let

$$
\Phi(y):=\frac{1}{\sqrt{2 \pi}} \int_{-\infty}^{y} e^{-\frac{x^{2}}{2}} d x
$$

be the cumulative distribution function of a standard Gaussian random variable and $\bar{\Phi}=1-\Phi$. We include a straightforward Lemma needed to estimate $N$.

Lemma 4.11. Let $C>0$ and $\bar{a} \in \mathbb{R}$. If $Y$ is a positive random variable such that $P(Y>t) \leq$ $\bar{\Phi}(\bar{a}+t / c)$, for every $t>a$, then

$$
E e^{s Y} \leq e^{a s}+e^{-c s \bar{a}+c^{2} s^{2} / 2} \quad \forall s>0 .
$$

Proof. We use elementary considerations and Fubini theorem, to obtain

$$
\begin{aligned}
E e^{s Y} & =\int_{0}^{\infty} P\left(e^{s Y}>t\right) d t=\int_{0}^{e^{s a}} P(Y>\log t / s) d t+\int_{e^{s a}}^{\infty} P(Y>\log t / s) d t \\
& \leq e^{s a}+\int_{0}^{\infty} \bar{\Phi}(\bar{a}+\log t / c s) d t=e^{s a}+\int_{0}^{\infty} \frac{1}{\sqrt{2 \pi}} \int_{a+\log t / c s} e^{-x^{2} / 2} d x d t \\
& =e^{s a}+\int_{\mathbb{R}} \int_{0}^{e^{c s(x-\bar{a})}} \frac{1}{\sqrt{2 \pi}} e^{-x^{2} / 2} d t d x=e^{s a}+\int_{\mathbb{R}} \frac{1}{\sqrt{2 \pi}} e^{c s(x-\bar{a})} e^{-x^{2} / 2} d x \\
& =e^{s a}+e^{-c s \bar{a}+c^{2} s^{2} / 2} .
\end{aligned}
$$

Theorem 4.12. Under the same assumptions of Lemma 4.10 the random variable $N$ defined in (55) has a Gaussian tail. Moreover, there exists $C=C(T, p)>0$, such that $C$ is bounded when $T$ is small and for all $s>1$,

$$
E e^{s N} \leq e^{C\left(\llbracket \sigma \rrbracket_{p}^{p}+1\right) L(\sigma, \beta, \mathbf{Z})^{p} s^{2}}
$$

where $L$ is defined in (36).

Proof. The main ingredient, which is still to prove, is that, for $P$-a.e. $\omega$,

$$
N_{1}\left(w_{\mathbf{F}},[0, T]\right)^{\frac{1}{q}}(\omega) \leq C \llbracket \sigma \rrbracket_{p}^{\frac{p}{q}}\left(g_{0, T}(\omega-h)^{\frac{p}{q}}+\llbracket h \rrbracket_{q,[0, T]}\right),
$$

where $g$ is defined as in (51) and $C:=C(p, q)$. The proof of this inequality follows from Lemma4.10 in the same way as the proof of [15, Lemma 11.12]. It follows from [15, Proposition 11.2], that we can take $q=1$, to obtain

$$
N_{1}\left(w_{\mathbf{F}},[0, T]\right)(\omega) \leq C \llbracket \sigma \rrbracket_{p}^{p}\left(g_{0, T}(\omega-h)+\llbracket h \rrbracket_{\mathcal{H}}\right),
$$


withe $C:=C(T, p)$. By assumption, $\sigma, \beta$ and $\mathbf{Z}$ are of finite $p$-variation. This implies that $g$ is almost surely finite and we can apply the generalized Fernique Theorem [15, Theorem 11.7] as follows. We set $f=N$ and $g=C \llbracket \sigma \rrbracket_{p}^{p} g^{p}$ defined as in (51). We must now find $a>0$ such that the following set has positive measure,

$$
A_{a}=\left\{\omega \in \Omega \mid C \llbracket \sigma \rrbracket_{p}^{p} g^{p}(\omega) \leq a\right\} .
$$

We know from Lemma 4.8 that $E\left[g^{p}\right]^{\frac{1}{p}} \leq C L(\sigma, \beta, \mathbf{Z})$. From Chebychev inequality, we have (where $C$ may change from a term to the next)

$$
P\left(g^{p} \geq a\left(C \llbracket \sigma \rrbracket_{p}^{p}\right)^{-1}\right) \leq \frac{C \llbracket \sigma \rrbracket_{p}^{p}}{a} E g^{p} \leq \frac{1}{a} C \llbracket \sigma \rrbracket_{p}^{p} L(\sigma, \beta, \mathbf{Z})^{p} .
$$

Using the previous estimates, we obtain that,

$$
P\left(A_{a}\right)=1-P\left(g^{p}>a\left(C \llbracket \sigma \rrbracket_{p}^{p}\right)^{-1}\right) \geq 1-P\left(g^{p} \geq a\left(C \llbracket \sigma \rrbracket_{p}^{p}\right)^{-1}\right) \geq 1-\frac{1}{a} C \llbracket \sigma \rrbracket_{p}^{p} L(\sigma, \beta, \mathbf{Z})^{p} .
$$

where $C=C(T, p)$ is again allowed to increase in the last inequality. Moreover,

$$
C(T, p) \rightarrow 0, \quad \text { as } T \rightarrow 0 .
$$

If we now fix $a=(C+1) \llbracket \sigma \rrbracket_{p}^{p} L(\sigma, \beta, \mathbf{Z})^{p}$, we have that $P\left(A_{a}\right) \geq 1-\frac{C}{C+1}>0$. From Fernique Theorem [15, Theorem 11.7], we have, for $r>a$,

$$
P(N>r) \leq \bar{\Phi}\left(\bar{a}+r\left(C \llbracket \sigma \rrbracket_{p}^{p}\right)^{-1}\right),
$$

where $\bar{a}=\hat{a}-a\left(C \llbracket \sigma \rrbracket_{p}^{p}\right)^{-1}$ and $\hat{a}=\Phi^{-1}\left(P\left(A_{a}\right)\right)$. By our choice of $a$ and the monotonicity of $\Phi^{-1}$, we have that $\hat{a} \geq \Phi^{-1}\left(1-\frac{C}{C+1}\right)$, which is a universal constant depending only on $(p, T)$, but can be negative. It follows from (56) that $\hat{a} \rightarrow \infty$ as $T \rightarrow 0$. We apply Lemma4.11 that, with $s>1$ (chosen so that $s \leq s^{2}$ ), $a$ and $\bar{a}$ as before and $c=C \llbracket \sigma \rrbracket_{p}^{p}$.

$$
\begin{aligned}
E e^{s N} & \leq e^{(C+1) \llbracket \sigma \rrbracket_{p}^{p} L(\sigma, \beta, \mathbf{Z})^{p} s}+e^{-C \llbracket \sigma \rrbracket_{p}^{p} s\left(\hat{a}-a\left(C \llbracket \sigma \rrbracket_{p}^{p}\right)^{-1}\right)+\left(C \llbracket \sigma \rrbracket_{p}^{p}\right)^{2} s^{2} / 2} \\
& \leq e^{(C+1) \llbracket \sigma \rrbracket_{p}^{p} L(\sigma, \beta, \mathbf{Z})^{p} s^{2}}+e^{C \llbracket \sigma \rrbracket_{p}^{p} s^{2}\left[\left(-\Phi^{-1}\left(1-\frac{C}{C+1}\right)+\frac{C+1}{C} L(\sigma, \beta, \mathbf{Z})^{p}\right)+C \llbracket \sigma \rrbracket_{p}^{p} / 2\right]} \\
& \leq e^{C\left(\llbracket \sigma \rrbracket_{p}^{p}+1\right) L(\sigma, \beta, \mathbf{Z})^{p} s^{2}} .
\end{aligned}
$$

The constant $C$ is allowed to change again in the last line, but one can easily see that it remains bounded, when $T$ is small enough.

\subsection{The average Itô formula}

In this section we prove a version of the Itô formula which we need to make the connection between (3) and (4). We note that at the present level of knowledge, we don't know how to make an $P$-a.s. Itô formula, but we only have the chain rule when we average over $\Omega$.

Proposition 4.13. Let $\left(\Omega, \mathcal{F},\left(\mathcal{F}_{t}\right)_{t \in[0, T]}, P\right)$ a complete filtered probability space and $W$ be a $d$ dimensional Wiener process on it. Let $\bar{\alpha} \in\left(\frac{1}{3}, \frac{1}{2}\right), \mathbf{Z} \in \mathscr{C}_{w g}^{\bar{\alpha}}\left([0, T], \mathbb{R}^{m}\right)$. Assume that $\sigma$ and $\beta$ satisfy Assumption 4.2 for $k>\frac{d}{2}+3$ and $\alpha \in\left(\frac{1}{3}, \bar{\alpha}\right)$. Let $\mathbf{F}$ be defined as in Lemma 4.8 .

Let $x(\xi)$ be the solution to equation (20) driven by $\mathbf{F}$ with initial condition $\xi \in \mathbb{R}^{d}$, in the sense of Definition 3.4 given by Proposition 3.13

Let $\Xi: \Omega \rightarrow \mathbb{R}^{d}$ be an $\mathcal{F}_{0}$-measurable random variable. Then the process $x_{t}(\Xi)$ is $\left(\mathcal{F}_{t}\right)_{t \geq 0}$ adapted. Moreover, $x$ is a random variable with values in $C^{\alpha}\left([0, T] ; \mathbb{R}^{d}\right)$. 
Proof. Let $t \in[0, T]$ and call $\mathbf{F}_{\mid[0, t]}$ the restriction of $\mathbf{F}$ on the interval $[0, t]$. We know from Proposition 3.11 that

$$
\mathbb{R}^{d} \times\left(C^{\alpha}\left([0, t] ; C_{b}^{3}\left(\mathbb{R}^{d} ; \mathbb{R}^{d}\right)\right) \times C_{2}^{2 \alpha}\left([0, t] ; C_{b}^{2}\left(\mathbb{R}^{d} \times \mathbb{R}^{d} ; \mathbb{R}^{d}\right)\right)\right) \rightarrow \mathbb{R}^{d}, \quad\left(\xi, \mathbf{F}_{[0, t]}\right) \mapsto x_{t},
$$

is a continuous mapping. Moreover the random variable $\left(\Xi, \mathbf{F}_{\mid[0, t]}\right)$ is $\mathcal{F}_{t}$-measurable. Hence,

$$
\omega \mapsto\left(\Xi, \mathbf{F}_{[0, t]}\right)(\omega) \mapsto x_{t}(\Xi)(\omega),
$$

is $\mathcal{F}_{t}$-measurable.

In a similar way we see that $x$ is a random variable in $C^{\alpha}\left([0, T] ; \mathbb{R}^{d}\right)$, since $\omega \mapsto \mathbf{F}(\omega)$ is measurable and $x$ is continuous w.r.t. the rough driver.

Proposition 4.14. Under the same assumptions as Proposition 4.13 let $x_{t}=x_{t}(\Xi)$. If $\phi \in C_{b}^{3} \otimes H^{k}$, endowed with the norm defined in (16), then

$$
E\left[\phi\left(x_{t}\right)\right]=E[\phi(\Xi)]+\int_{0}^{t} \frac{1}{2} E\left[\nabla_{1}^{2} \phi\left(x_{r}\right)\left(\sigma_{r}\left(x_{r}\right) \sigma_{r}\left(x_{r}\right)^{T}\right)\right] d r+\int_{0}^{t} E\left[\nabla_{1} \phi\left(x_{r}\right) \beta_{r}\left(x_{r}\right)\right] d \mathbf{Z}_{r} \in H^{k},
$$

where $E\left[\nabla_{1} \phi\left(x_{r}\right) \beta_{r}\left(x_{r}\right)\right] \in \mathcal{L}\left(\mathbb{R}^{m} ; H^{k}\right)$ is controlled by $Z$ with Gubinelli derivative $E\left[\nabla_{1} \phi\left(x_{r}\right)\left(\beta_{r}^{\prime}\left(x_{r}\right)+\right.\right.$ $\left.\left.\nabla_{1} \beta_{r}\left(x_{r}\right) \beta_{r}\left(x_{r}\right)\right)+\nabla_{1}^{2} \phi\left(x_{r}\right) \beta_{r}\left(x_{r}\right) \otimes \beta_{r}\left(x_{r}\right)\right]$.

Before we proceed with the proof of Proposition 4.14, we prove two technical lemmas.

Lemma 4.15. Under the same assumptions as Proposition 4.13, let $x^{\sharp}$ be defined in (23). For any $\rho \in \mathbb{N}$ and $|t-s| \leq h \leq T$, we have

$$
E\left[\left|x_{s t}^{\sharp}\right|^{\rho}\right] \leq C\left(h^{\rho} \vee h^{\frac{\rho}{2}}\right)^{\bar{\alpha}-\alpha}|t-s|^{2 \alpha \rho} L^{\rho},
$$

where $L:=L(\sigma, \beta, \mathbf{Z})$ is defined in (36).

Proof. Define the random variable $Y:=C\|\mathbf{F}\|_{\alpha, h ; C^{3}}$ as in Proposition 3.7 which gives that for $|t-s|^{\alpha} \leq$ $Y^{-1}$ we have $\left|x_{s t}^{\sharp}\right| \leq Y|t-s|^{2 \alpha}$. Writing $\Omega=\left\{|t-s|^{\alpha} Y>1\right\} \cup\left\{|t-s|^{\alpha} Y \leq 1\right\}$ gives

$$
\begin{aligned}
E\left[\left|x_{s t}^{\sharp}\right|^{\rho}\right] & =E\left[1_{|t-s|^{\alpha} Y>1}\left|x_{s t}^{\#}\right|^{\rho}\right]+E\left[1_{|t-s|^{\alpha} Y \leq 1}\left|x_{s t}^{\sharp}\right|^{\rho}\right] \leq|t-s|^{\rho \alpha} E\left[Y^{\rho}\left|x_{s t}^{\sharp}\right|^{\rho}\right]+E\left[Y^{\rho}\right]|t-s|^{\rho 2 \alpha} \\
& \leq|t-s|^{\rho \alpha} E\left[Y^{2 \rho}\right]^{1 / 2} E\left[\left|x_{s t}^{\sharp}\right|^{2 \rho}\right]^{1 / 2}+E\left[Y^{\rho}\right]|t-s|^{\rho 2 \alpha} .
\end{aligned}
$$

Now trivially by the definition of $x^{\sharp}$, we have

$$
\left|x_{s t}^{\sharp}\right| \leq\left([x]_{\alpha, h}+\|F\|_{\alpha, h ; C}\right)|t-s|^{\alpha} \leq C|| \mathbf{F} \|_{\alpha, h ; C^{3}}|t-s|^{\alpha}, \quad P-a . s .
$$

and the result follows from Lemma4.8.

Lemma 4.16. Under the same assumptions as Proposition 4.13 we have

$$
\left(E[\phi(x)], E\left[\nabla_{1} \phi(x) \beta(x)\right]\right) \in \mathscr{D}_{Z}^{2 \alpha}\left([0, T] ; H^{k}\right),
$$

with bounds, on a time interval of size $h \leq T$,

$$
\left\|\left(E[\phi(x)], E\left[\nabla_{1} \phi(x) \beta(x)\right]\right)\right\|_{Z, \alpha, h ; H^{k}} \leq L\left(h \vee h^{\frac{1}{2}}\right)^{\bar{\alpha}-\alpha}\|\phi\|_{C_{b}^{3} \otimes H^{k}}\left(1+\left\|[x]_{\alpha, h}\right\|_{L_{\omega}^{2}}\right),
$$

where $L:=L(\sigma, \beta, \mathbf{Z})$ is defined in (36). 
Proof. We do a first order Taylor expansion to obtain

$$
\begin{aligned}
\delta \phi(x)_{s t} & =\left[\nabla_{1} \phi\right]_{s t}^{1, x} \delta x_{s t}=\left[\nabla_{1} \phi\right]_{s t}^{1, x} W_{s t}^{\sigma}\left(x_{s}\right)+\left[\nabla_{1} \phi\right]_{s t}^{1, x} Z_{s t}^{\beta}\left(x_{s}\right)+\left[\nabla_{1} \phi\right]_{s t}^{1, x} x_{s t}^{\#} \\
& =\nabla_{1} \phi\left(x_{s}\right) \beta_{s}^{j}\left(x_{s}\right) Z_{s t}^{j}+\phi(x)_{s t}^{\#}, \quad P-a . s .
\end{aligned}
$$

We have defined

$$
\phi(x)_{s t}^{\sharp}:=\left[\nabla_{1} \phi\right]_{s t}^{1, x} W_{s t}^{\sigma}\left(x_{s}\right)+\left[\nabla_{1} \phi\right]_{s t}^{1, x} x_{s t}^{\sharp}+\left[\nabla_{1} \phi\right]_{s t}^{1, x} Z_{s t}^{\beta}\left(x_{s}\right)-\nabla_{1} \phi\left(x_{s}\right) \beta_{s}^{j}\left(x_{s}\right) Z_{s t}^{j} .
$$

We first make some deterministic bounds (i.e. uniformly in $\omega$ )

$$
\begin{aligned}
\|\left[\nabla_{1} \phi\right]_{s t}^{1, x} Z_{s t}^{\beta}\left(x_{s}\right) & -\nabla_{1} \phi\left(x_{s}\right) \beta_{s}^{j}\left(x_{s}\right) Z_{s t}^{j} \|_{H^{k}} \\
& \left.\leq\left\|\left(\left[\nabla_{1} \phi\right]_{s t}^{1, x}-\nabla_{1} \phi\left(x_{s}\right)\right) Z_{s t}^{\beta}\left(x_{s}\right)\right\|_{H^{k}}+\| \nabla_{1} \phi\left(x_{s}\right)\right)\left(Z_{s t}^{\beta}\left(x_{s}\right)-\beta_{s}^{j}\left(x_{s}\right)\right) Z_{s t}^{j} \|_{H^{k}} \\
& \leq\|\phi\|_{C_{b}^{3} \otimes H^{k}}[x]_{\alpha, h}\left[Z^{\beta}\right]_{\alpha, h ; C_{b}}|t-s|^{2 \alpha}+\|\phi\|_{C_{b}^{3} \otimes H^{k}}\left[\left(Z^{\beta}\right)^{\sharp}\right]_{2 \alpha, h}|t-s|^{2 \alpha} \\
& \leq\|\phi\|_{C_{b}^{3} \otimes H^{k}}\left\|\mathbf{Z}^{\beta}\right\|_{\alpha, h ; C_{b}^{3}}\left(1+[x]_{\alpha, h}\right)|t-s|^{2 \alpha} .
\end{aligned}
$$

Using that $x$ is adapted we get $E\left[\nabla_{1} \phi\left(x_{s}\right) W_{s t}^{\sigma}\left(x_{s}\right)\right]=0$ so that

$$
\begin{aligned}
\left\|E\left[\left[\nabla_{1} \phi\right]_{s t}^{1, x} W_{s t}^{\sigma}\left(x_{s}\right)\right]\right\|_{H^{k}} & =\left\|E\left[\left[\nabla_{1}^{2} \phi\right]_{s t}^{2, x} \delta x_{s t} W_{s t}^{\sigma}\left(x_{s}\right)\right]\right\|_{H^{k}} \leq\|\phi\|_{C_{b}^{3} \otimes H^{k}}\left\|[x]_{\alpha, h}\right\|_{L_{\omega}^{2}}\|\sigma\|_{L_{t}^{\infty} \mathcal{L}\left(\mathbb{R}^{m} ; H^{k}\right)}|t-s|^{\alpha+\frac{1}{2}} \\
& \leq h^{\frac{1}{2}-\alpha}\|\phi\|_{C_{b}^{3} \otimes H^{k}}\left\|[x]_{\alpha, h}\right\|_{L_{\omega}^{2}}\|\sigma\|_{L_{t}^{\infty} \mathcal{L}\left(\mathbb{R}^{m} ; H^{k}\right)}|t-s|^{2 \alpha} .
\end{aligned}
$$

Write now

$$
\left\|E\left[\left[\nabla_{1} \phi\right]_{s t}^{1, x} x_{s t}^{\sharp}\right]\right\|_{H^{k}} \leq\|\phi\|_{C_{b}^{3} \otimes H^{k}} E\left[\left|x_{s t}^{\sharp}\right|\right],
$$

and the result follows from Lemma 4.15 with $\rho=1$.

Proof of Proposition 4.14 We do a third order Taylor expansion to obtain, $P$-a.s.,

$$
\begin{aligned}
\delta \phi(x)_{s t}= & \nabla_{1} \phi\left(x_{s}\right) \delta x_{s t}+\frac{1}{2} \nabla_{1}^{2} \phi\left(x_{s}\right)\left(\delta x_{s t}\right)^{2}+\left[\nabla_{1}^{3} \phi\right]_{s t}^{3, x}\left(\delta x_{s t}\right)^{3} \\
= & \nabla_{1} \phi\left(x_{s}\right) W_{s t}^{\sigma}\left(x_{s}\right)+\nabla_{1} \phi\left(x_{s}\right) W_{s t}^{\sigma}\left(x_{s}\right)+\nabla_{1} \phi\left(x_{s}\right) Z_{s t}^{\beta}\left(x_{s}\right)+\nabla_{1} \phi\left(x_{s}\right) \mathbb{Z}_{s t}^{\beta}\left(x_{s}\right) \\
& +\nabla_{1} \phi\left(x_{s}\right) \int_{s}^{t} Z_{s r}^{\beta}\left(x_{s}\right) \nabla_{r}\left(x_{s}\right) d W_{r}+\nabla_{1} \phi\left(x_{s}\right) \int_{s}^{t} W_{s r}^{\sigma}\left(x_{s}\right) \nabla \beta_{r}\left(x_{s}\right) d \mathbf{Z}_{r} \\
& +\frac{1}{2} \nabla_{1}^{2} \phi\left(x_{s}\right)\left(W_{s t}^{\sigma}\left(x_{s}\right)\right)^{\otimes 2}+\frac{1}{2} \nabla_{1}^{2} \phi\left(x_{s}\right)\left(x_{s t}^{\sharp}\right)^{\otimes 2}+\nabla_{1}^{2} \phi\left(x_{s}\right)\left(W_{s t}^{\sigma}\left(x_{s}\right) \otimes x_{s t}^{\sharp}\right) \\
& +\nabla_{1}^{2} \phi\left(x_{s}\right)\left(Z_{s t}^{\beta}\left(x_{s}\right) \otimes x_{s t}^{\sharp}\right)+\frac{1}{2} \nabla_{1}^{2} \phi\left(x_{s}\right)\left(Z_{s t}^{\beta}\left(x_{s}\right) \otimes Z_{s t}^{\beta}\left(x_{s}\right)\right) \\
& +\nabla_{1} \phi\left(x_{s}\right) x_{s t}^{\natural}+\left[\nabla_{1}^{3} \phi\right]_{s t}^{3, x}\left(\delta x_{s t}\right)^{\otimes 3} \\
= & \frac{1}{2} \int_{s}^{t} \nabla_{1}^{2} \phi\left(x_{r}\right)\left(\sigma_{r}\left(x_{r}\right) \sigma_{r}\left(x_{r}\right)^{T}\right) d r+\nabla_{1} \phi\left(x_{s}\right) \beta_{s}^{j}\left(x_{s}\right) Z_{s t}^{j} \\
& +\left(\nabla_{1} \phi\left(x_{s}\right)\left(\beta_{s}^{j, i}\left(x_{s}\right)+\nabla_{1} \beta_{s}^{j}\left(x_{s}\right) \beta_{s}^{i}\left(x_{s}\right)\right)+\nabla_{1}^{2} \phi\left(x_{s}\right)\left(\beta^{j}\left(x_{s}\right) \otimes \beta^{i}\left(x_{s}\right)\right)\right) \mathbb{Z}_{s t}^{i, j}+\phi(x)_{s t}^{\natural} .
\end{aligned}
$$


Where we have defined

$$
\begin{aligned}
\phi(x)_{s t}^{\natural}= & \nabla_{1} \phi\left(x_{s}\right) W_{s t}^{\sigma}\left(x_{s}\right)+\nabla_{1} \phi\left(x_{s}\right) \mathbb{W}_{s t}^{\sigma}\left(x_{s}\right)+\nabla_{1} \phi\left(x_{s}\right) \int_{s}^{t} Z_{s r}^{\beta}\left(x_{s}\right) \nabla \sigma_{r}\left(x_{s}\right) d W_{r} \\
& +\nabla_{1} \phi\left(x_{s}\right) \int_{s}^{t} W_{s r}^{\sigma}\left(x_{s}\right) \nabla \beta_{r}\left(x_{s}\right) d \mathbf{Z}_{r}+\frac{1}{2} \nabla_{1}^{2} \phi\left(x_{s}\right) \int_{s}^{t} \sigma_{r}\left(x_{s}\right) \sigma_{r}\left(x_{s}\right)^{T} d r-\frac{1}{2} \nabla_{1}^{2} \phi\left(x_{s}\right)\left(W_{s t}^{\sigma}\left(x_{s}\right)\right)^{\otimes 2} \\
& +\frac{1}{2} \int_{s}^{t}\left(\nabla_{1}^{2} \phi\left(x_{r}\right)-\nabla_{1}^{2} \phi\left(x_{s}\right)\right)\left(\sigma_{r}\left(x_{s}\right) \sigma_{r}\left(x_{s}\right)^{T}\right) d r \\
& +\frac{1}{2} \int_{s}^{t} \nabla_{1}^{2} \phi\left(x_{r}\right)\left(\sigma_{r}\left(x_{r}\right) \sigma_{r}\left(x_{r}\right)^{T}-\sigma_{r}\left(x_{s}\right) \sigma_{r}\left(x_{s}\right)^{T}\right) d r \\
& +\nabla_{1} \phi\left(x_{s}\right) Z_{s t}^{\beta}\left(x_{s}\right)-\nabla_{1} \phi\left(x_{s}\right) \beta_{s}^{j}\left(x_{s}\right) Z_{s t}^{j} \\
& +\nabla_{1} \phi\left(x_{s}\right) \mathbb{Z}_{s t}^{\beta}\left(x_{s}\right)-\nabla_{1} \phi\left(x_{s}\right)\left(\beta_{s}^{j, i}\left(x_{s}\right)+\nabla \beta_{s}^{j}\left(x_{s}\right) \beta_{s}^{i}\left(x_{s}\right)\right) \mathbb{Z}_{s t}^{i, j} \\
& +\frac{1}{2} \nabla_{1}^{2} \phi\left(x_{s}\right)\left(Z_{s t}^{\beta}\left(x_{s}\right) \otimes Z_{s t}^{\beta}\left(x_{s}\right)\right)-\nabla_{1}^{2} \phi\left(x_{s}\right)\left(\beta_{s}^{j}\left(x_{s}\right) \otimes \beta_{s}^{i}\left(x_{s}\right)\right) \mathbb{Z}_{s t}^{i, j} \\
& +\frac{1}{2} \nabla_{1}^{2} \phi\left(x_{s}\right)\left(x_{s t}^{\sharp}\right)^{\otimes 2}+\nabla_{1}^{2} \phi\left(x_{s}\right)\left(W_{s t}^{\sigma}\left(x_{s}\right) \otimes x_{s t}^{\sharp}\right) \\
& +\nabla_{1}^{2} \phi\left(x_{s}\right)\left(Z_{s t}^{\beta}\left(x_{s}\right) \otimes x_{s t}^{\sharp}\right)+\frac{1}{2} \nabla_{1}^{2} \phi\left(x_{s}\right)\left(Z_{s t}^{\beta}\left(x_{s}\right) \otimes Z_{s t}^{\beta}\left(x_{s}\right)\right) \\
& +\nabla_{1} \phi\left(x_{s}\right) x_{s t}^{\natural}+\left[\nabla_{1}^{3} \phi\right]_{s t}^{3, x}\left(\delta x_{s t}\right)^{\otimes 3} .
\end{aligned}
$$

As in Lemma 4.7 we note that

$$
\nabla_{1} \phi\left(x_{s}\right) Z_{s t}^{\beta}\left(x_{s}\right)-\nabla_{1} \phi\left(x_{s}\right) \beta_{s}^{j}\left(x_{s}\right) Z_{s t}^{j}+\nabla_{1} \phi\left(x_{s}\right) \mathbb{Z}_{s t}^{\beta}\left(x_{s}\right)-\nabla_{1} \phi\left(x_{s}\right)\left(\beta_{s}^{j, i}\left(x_{s}\right)+\nabla \beta_{s}^{j}\left(x_{s}\right) \beta_{s}^{i}\left(x_{s}\right)\right) \mathbb{Z}_{s t}^{i, j}
$$

is uniformly in $\omega$ bounded by $|t-s|^{3 \alpha}$ depending only on $\beta$. Moreover, since $\mathbf{Z}$ is geometric and $\nabla^{2} \phi$ is a symmetric bilinear mapping we get

$$
\begin{aligned}
\frac{1}{2} \nabla_{1}^{2} \phi\left(x_{s}\right)\left(Z_{s t}^{\beta}\left(x_{s}\right)\right. & \left.\otimes Z_{s t}^{\beta}\left(x_{s}\right)\right)-\nabla_{1}^{2} \phi\left(x_{s}\right) \beta\left(x_{s}\right) \otimes \beta\left(x_{s}\right) \mathbb{Z}_{s t} \\
& \left.\left.=\frac{1}{2} \nabla_{1}^{2} \phi\left(x_{s}\right)\left(\left(Z_{s t}^{\beta}\right)^{\sharp}\left(x_{s}\right) \otimes\left(Z_{s t}^{\beta}\right)^{\sharp}\left(x_{s}\right)\right)\right)+\nabla_{1}^{2} \phi\left(x_{s}\right)\left(Z_{s t}^{\beta}\right)^{\sharp}\left(x_{s}\right) \hat{\otimes} \beta_{s}\left(x_{s}\right) Z_{s t}\right)
\end{aligned}
$$

where $\hat{\otimes}$ denotes the symmetric tensor product. This is clearly bounded by $|t-s|^{3 \alpha}$. result.

Using Lemma 4.15 with $\rho=1$ and $\rho=2$ and taking the expectation of $\phi(x)_{s t}^{\natural}$ we obtain the

To create the contraction mapping in the appropriate space of measures we shall need to control the difference of two measures induced by two rough SDEs.

Proposition 4.17. Let $\left(\Omega, \mathcal{F},\left(\mathcal{F}_{t}\right)_{t \in[0, T]}, P\right)$ a complete filtered probability space and $W$ be a $d$ dimensional Wiener process on it. Let $\bar{\alpha} \in\left(\frac{1}{3}, \frac{1}{2}\right), \mathbf{Z} \in \mathscr{C}_{w g}^{\bar{\alpha}}\left([0, T], \mathbb{R}^{m}\right)$. Assume that $(\sigma, \beta)$ and $(\theta, \gamma)$ satisfy Assumption 4.2 for $k>\frac{d}{2}+3, \alpha \in\left(\frac{1}{3}, \bar{\alpha}\right)$ and $p=\frac{1}{\alpha}$. Let $\mathbf{F}$ and $\mathbf{G}$ be nonlinear rough drivers constructed from $F_{s t}:=W_{s t}^{\sigma}+Z_{s t}^{\beta}$ and $G_{s t}:=W_{s t}^{\theta}+Z_{s t}^{\gamma}$ as in Lemma 4.8 Moreover, let $\Xi$ be an $\mathcal{F}_{0}$-measurable random variable.

Let $x$ and $y$ solutions to equation (20) driven by $\mathbf{F}$ and $\mathbf{G}$ respectively, with the same initial condition $\Xi$. 
If $\phi \in C_{b}^{3} \otimes H^{k}$, endowed with the norm defined in (16), we have

$$
\left(E[\phi(x)-\phi(y)], E\left[\nabla_{1} \phi(x) \beta(x)-\nabla_{1} \phi(y) \gamma(y)\right]\right) \in \mathscr{D}_{Z}^{2 \alpha}\left([0, T] ; H^{k}\right) .
$$

Moreover, there exists $\rho \geq 1$ and $C(T)$ such that $\lim _{T \rightarrow 0} C(T)=0$, and

$$
\begin{gathered}
\left\|\left(E[\phi(x)-\phi(y)], E\left[\nabla_{1} \phi(x) \beta(x)\right]\right)-\left(E[\phi(y)], E\left[\nabla_{1} \phi(y) \gamma(y)\right]\right)\right\|_{Z, \alpha ; H^{k}} \\
\leq C(T) e^{M^{\bar{\rho}}}\|\phi\|_{C_{b}^{3} \otimes H^{k}}\left(\|\sigma-\theta\|_{L_{t}^{\infty} \mathcal{L}\left(\mathbb{R}^{d} ; H^{k}\right)}+\left\|\left(\beta, \beta^{\prime}\right)-\left(\gamma, \gamma^{\prime}\right)\right\|_{Z, \alpha ; H^{k}}\right) .
\end{gathered}
$$

where $M:=K\left(\llbracket \sigma \rrbracket_{p,[s, t]}^{\rho}+1\right)(L(\sigma, \beta, \mathbf{Z})+L(\theta, \gamma, \mathbf{Z}))$, L is defined in (36) and $K=K(\alpha, \rho)>0$ is a universal constant.

Before proceeding with the proof, we need the next two technical lemmas.

Lemma 4.18. Under the same assumptions of Proposition 4.17 for any $\rho \geq 1$, there exists $\bar{\rho} \geq \rho, C$ and $C(T)>0$, such that $\lim _{T \rightarrow 0} C(T)=0$ and

$$
\left\|[x-y]_{\alpha}\right\|_{L_{\omega}^{\rho}} \leq C(T) e^{M^{\bar{\rho}}}\left(\|\sigma-\theta\|_{L_{t}^{\infty} \mathcal{L}\left(\mathbb{R}^{d} ; H^{k}\right)}+\left\|\left(\beta, \beta^{\prime}\right)-\left(\gamma, \gamma^{\prime}\right)\right\|_{Z, \alpha ; H^{k}}\right) .
$$

Proof. By applying Corollary 3.12, (50) and (49), we see that there exists $\bar{\rho} \geq 1$ and $K_{\bar{\rho}} \in L^{\bar{\rho}}(\Omega)$ such that $P$-a.s.,

$$
\begin{aligned}
{[x-y]_{\alpha} } & \leq C e^{C N\left(w_{\mathbf{F}},[0, T]\right)}[\mathbf{F}-\mathbf{G}]_{\alpha}\left(1+[\mathbf{F}]_{\alpha}+[\mathbf{G}]_{\alpha}\right)^{2}\left(\left([\mathbf{F}]_{\alpha}+[\mathbf{G}]_{\alpha}\right) \vee\left([\mathbf{F}]_{\alpha}+[\mathbf{G}]_{\alpha}\right)^{\frac{1}{\alpha}}\right) \\
& \leq C e^{C N\left(w_{\mathbf{F}},[0, T]\right)} T^{\bar{\alpha}-\alpha} K_{\rho} M^{\rho}\left(\|\sigma-\theta\|_{L_{t}^{\infty} H^{k}}+\left\|\left(\beta, \beta^{\prime}\right)-\left(\gamma, \gamma^{\prime}\right)\right\|_{Z, \alpha ; H^{k}}\right) .
\end{aligned}
$$

Taking the $L_{\omega}^{\rho}$ norm on both sides we conclude the proof, thanks to Theorem 4.12 , which gives

$$
E e^{C N\left(w_{\mathbf{F}},[0, T]\right)} \leq e^{C\left(\llbracket \sigma \rrbracket_{p}^{p}+1\right) L(\sigma, \beta, \mathbf{Z})^{p}},
$$

where $C>0$ is a universal constant.

Lemma 4.19. Under the same assumptions of Proposition 4.17 for any $\rho \geq 1$, there exists $\bar{\rho} \geq \rho$ and $C(T)>0$, such that $\lim _{T \rightarrow 0} C(T)=0$ and, for all $s, t \in[0, T]$,

$$
\left\|x_{s t}^{\#}-y_{s t}^{\sharp}\right\|_{L_{\omega}^{\rho}} \leq C(T) e^{M^{\bar{\rho}}}\left(\|\sigma-\theta\|_{L_{t}^{\infty} \mathcal{L}\left(\mathbb{R}^{m}, H^{k}\right)}+\left\|\left(\beta, \beta^{\prime}\right)-\left(\gamma, \gamma^{\prime}\right)\right\|_{Z, \alpha ; H^{k}}\right)|t-s|^{2 \alpha} .
$$

Proof. Let $Y:=C\left([\mathbf{F}]_{\alpha}+[\mathbf{G}]_{\alpha}\right)\left(1+[\mathbf{F}]_{\alpha}+[\mathbf{G}]_{\alpha}\right)^{2}$ where $C$ is the constant given in Proposition 3.11. Then, $|t-s|^{\alpha} Y \leq 1$ implies,

$$
\left|x_{s t}^{\sharp}-y_{s t}^{\sharp}\right| \leq|t-s|^{2 \alpha} Y e^{C N\left(w_{\mathbf{F}},[0, T]\right)}[\mathbf{F}-\mathbf{G}]_{\alpha}
$$

and we notice that $E\left[Y^{\rho}\right] \leq M^{\bar{\rho}}\left(T^{\rho} \vee T^{\frac{\rho}{2}}\right)^{\bar{\alpha}-\alpha}$ for some $\bar{\rho} \geq \rho \geq 1$ which follows from Lemma 4.8 and the Gaussian integrability of $N\left(w_{\mathbf{F}},[0, T]\right)$, Theorem 4.12 .

We split up $\Omega=\left\{|t-s|^{\alpha} Y \leq 1\right\} \cup\left\{|t-s|^{\alpha} Y>1\right\}$ which gives

$$
E\left[\left|x_{s t}^{\sharp}-y_{s t}^{\sharp}\right|^{\rho}\right] \leq|t-s|^{2 \rho \alpha} E\left[Y^{2 \rho}\left|x_{s t}^{\sharp}-y_{s t}^{\sharp}\right|^{\rho}\right]+|t-s|^{2 \rho \alpha} E\left[Y^{\rho} e^{C N\left(w_{\mathbf{F}},[0, T]\right)}[\mathbf{F}-\mathbf{G}]_{\alpha, h}^{\rho}\right] .
$$

For the first term above we use the crude (in time) bound

$$
\begin{aligned}
\left|x_{s t}^{\sharp}-y_{s t}^{\sharp}\right| & \leq[x-y]_{\alpha, h}|t-s|^{\alpha}+[\mathbf{F}]_{\alpha, h}\left|x_{s}-y_{s}\right| t-\left.s\right|^{\alpha}+[\mathbf{F}-\mathbf{G}]_{\alpha, h}|t-s|^{\alpha} \\
& \leq C(T) Y e^{C N\left(w_{\mathbf{F}},[0, T]\right)}[\mathbf{F}-\mathbf{G}]_{\alpha} .
\end{aligned}
$$

The result follows from Corollary 3.12, (29) and Theorem 4.12, 
Proof of Proposition 4.17 We write

$$
\begin{aligned}
\phi(x)_{s t}^{\sharp}-\phi(y)_{s t}^{\#} & :=\left[\nabla_{1} \phi\right]_{s t}^{1, x} W_{s t}^{\sigma}\left(x_{s}\right)-\left[\nabla_{1} \phi\right]_{s t}^{1, y} W_{s t}^{\theta}\left(y_{s}\right)+\left[\nabla_{1} \phi\right]_{s t}^{1, x} x_{s t}^{\sharp}-\left[\nabla_{1} \phi\right]_{s t}^{1, y} y_{s t}^{\#} \\
& +\left[\nabla_{1} \phi\right]_{s t}^{1, x} Z_{s t}^{\beta}\left(x_{s}\right)-\nabla_{1} \phi\left(x_{s}\right) \beta_{s}^{j}\left(x_{s}\right) Z_{s t}^{j}-\left[\nabla_{1} \phi\right]_{s t}^{1, y} Z_{s t}^{\gamma}\left(y_{s}\right)+\nabla_{1} \phi\left(y_{s}\right) \gamma_{s}^{j}\left(y_{s}\right) Z_{s t}^{j} .
\end{aligned}
$$

We start from the first term on the right hand side of (57),

$$
\begin{aligned}
{\left[\nabla_{1} \phi\right]_{s t}^{1, x} W_{s t}^{\sigma}\left(x_{s}\right)-} & {\left[\nabla_{1} \phi\right]_{s t}^{1, y} W_{s t}^{\theta}\left(y_{s}\right) } \\
= & {\left[\nabla_{1}^{2} \phi\right]_{s t}^{2, x} \delta x_{s t} W_{s t}^{\sigma}\left(x_{s}\right)-\left[\nabla_{1}^{2} \phi\right]_{s t}^{2, y} \delta y_{s t} W_{s t}^{\theta}\left(y_{s}\right)+\nabla_{1} \phi\left(x_{s}\right) W_{s t}^{\sigma}\left(x_{s}\right)-\nabla_{1} \phi\left(y_{s}\right) W_{s t}^{\theta}\left(y_{s}\right) } \\
= & \left(\left[\nabla_{1}^{2} \phi\right]_{s t}^{2, x}-\left[\nabla_{1}^{2} \phi\right]_{s t}^{2, y}\right) \delta x_{s t} W_{s t}^{\sigma}\left(x_{s}\right)+\left[\nabla_{1}^{2} \phi\right]_{s t}^{2, y}\left(\delta x_{s t}-\delta y_{s t}\right) W_{s t}^{\sigma}\left(x_{s}\right) \\
& +\left[\nabla_{1}^{2} \phi\right]_{s t}^{2, y} \delta y_{s t}\left(W_{s t}^{\sigma}\left(x_{s}\right)-W_{s t}^{\theta}\left(x_{s}\right)\right)+\left[\nabla_{1}^{2} \phi\right]_{s t}^{2, y} \delta y_{s t}\left(W_{s t}^{\theta}\left(x_{s}\right)-W_{s t}^{\theta}\left(y_{s}\right)\right) .
\end{aligned}
$$

We have, as an application of Hölder inequality, for $0 \leq s \leq t \leq T$,

$$
\begin{aligned}
\| E & {\left[\left(\left[\nabla_{1}^{2} \phi\right]_{s t}^{2, x}-\left[\nabla_{1}^{2} \phi\right]_{s t}^{2, y}\right) \delta x_{s t} W_{s t}^{\sigma}\left(x_{s}\right)\right]\left\|_{H^{k}} \leq\right\| \phi \|_{C_{b}^{3} \otimes H^{k}} E\left[\|x-y\|_{L_{t}^{\infty}}\left|\delta x_{s t}\right|\left|W_{s t}^{\sigma}\left(x_{s}\right)\right|\right] } \\
& \leq\|\phi\|_{C_{b}^{3} \otimes H^{k}}\|x-y\|_{L_{\omega}^{2} L_{t}^{\infty}}\left\|\delta x_{s t}\right\|_{L_{\omega}^{4}}\left\|W_{s t}^{\sigma}\left(x_{s}\right)\right\|_{L_{\omega}^{4}} \\
& \leq C(T)\|\phi\|_{C_{b}^{3} \otimes H^{k}}\|x-y\|_{L_{\omega}^{2} L_{t}^{\infty}}\left\|[x]_{\alpha}\right\|_{L_{\omega}^{4}}\|\sigma\|_{L_{t}^{\infty} \mathcal{L}\left(\mathbb{R}^{d} ; H^{k}\right)}|t-s|^{2 \alpha} .
\end{aligned}
$$

where, in the last inequality we used Lemma 4.3. Similarly, using Lemma 4.3 and 4.4, we can bound the remaining terms,

$$
\left\|E\left[\left[\nabla_{1}^{2} \phi\right]_{s t}^{2, y}\left(\delta x_{s t}-\delta y_{s t}\right) W_{s t}^{\sigma}\left(x_{s}\right)\right]\right\|_{H^{k}} \leq C(T)\|\phi\|_{C_{b}^{3} \otimes H^{k}}\left\|[x-y]_{\alpha}\right\|_{L_{\omega}^{2}}\|\sigma\|_{L_{t}^{\infty} \mathcal{L}\left(\mathbb{R}^{d} ; H^{k}\right)}|t-s|^{2 \alpha},
$$$$
\left\|E\left[\left[\nabla_{1}^{2} \phi\right]_{s t}^{2, y} \delta y_{s t}\left(W_{s t}^{\sigma}\left(x_{s}\right)-W_{s t}^{\theta}\left(x_{s}\right)\right)\right]\right\|_{H^{k}} \leq C(T)\|\phi\|_{C_{b}^{3} \otimes H^{k}}\left\|[y]_{\alpha}\right\|_{L_{\omega}^{2}}\left(1+\|\sigma\|_{L_{t}^{\infty} \mathcal{L}\left(\mathbb{R}^{d} ; H^{k}\right)}+\|\theta\|_{L_{t}^{\infty} \mathcal{L}\left(\mathbb{R}^{d} ; H^{k}\right)}\right)
$$

$$
\cdot\|\sigma-\theta\|_{L_{t}^{\infty} \mathcal{L}\left(\mathbb{R}^{d} ; H^{k}\right)}|t-s|^{2 \alpha} .
$$

$\left\|E\left[\left[\nabla_{1}^{2} \phi\right]_{s t}^{2, y} \delta y_{s t}\left(W_{s t}^{\theta}\left(x_{s}\right)-W_{s t}^{\theta}\left(y_{s}\right)\right)\right]\right\|_{H^{k}} \leq C(T)\|\phi\|_{C_{b}^{3} \otimes H^{k}}\|x-y\|_{L_{\omega}^{2} L_{t}^{\infty}}\left\|[y]_{\alpha}\right\|_{L_{\omega}^{4}}\|\sigma\|_{L_{t}^{\infty} \mathcal{L}\left(\mathbb{R}^{d} ; H^{k}\right)}|t-s|^{2 \alpha}$.

Summing up the previous inequalities, we get

$$
\begin{aligned}
&\left\|E\left[\left[\nabla_{1} \phi\right]_{s t}^{1, x} W_{s t}^{\sigma}\left(x_{s}\right)-\left[\nabla_{1} \phi\right]_{s t}^{1, y} W_{s t}^{\theta}\left(y_{s}\right)\right]\right\|_{H^{k}} \leq M C(T)\|\phi\|_{C_{b}^{3} \otimes H^{k}}|t-s|^{2 \alpha}\left(1+\left\|[x]_{\alpha}\right\|_{L_{\omega}^{4}}+\left\|[y]_{\alpha}\right\|_{L_{\omega}^{4}}\right) \\
& \cdot\left(\|x-y\|_{L_{\omega}^{2} L_{t}^{\infty}}+\left\|[x-y]_{\alpha}\right\|_{L_{\omega}^{2}}+\|\sigma-\theta\|_{L_{t}^{\infty} \mathcal{L}\left(\mathbb{R}^{d} ; H^{k}\right)}\right) .
\end{aligned}
$$

The second term in (57) is bounded as follows using Lemmas 4.19 and 4.15 ,

$$
\begin{aligned}
& \left\|E\left[\left[\nabla_{1} \phi\right]_{s t}^{1, x} x_{s t}^{\sharp}-\left[\nabla_{1} \phi\right]_{s t}^{1, y} y_{s t}^{\sharp}\right]\right\|_{H^{k}} \leq\|\phi\|_{C_{b}^{3} \otimes H^{k}}\left[\left\|x_{s t}^{\sharp}\right\|_{L_{\omega}^{2}}\|x-y\|_{L_{\omega}^{2} L_{t}^{\infty}}+\left\|x_{s t}^{\sharp}-y_{s t}^{\sharp}\right\|_{L_{\omega}^{1}}\right] \\
& \quad \leq C(T)\|\phi\|_{C_{b}^{3} \otimes H^{k}} e^{M^{\bar{\rho}}}\left(\|x-y\|_{L_{\omega}^{2} L_{t}^{\infty}}+\|\sigma-\theta\|_{L_{t}^{\infty} \mathcal{L}\left(\mathbb{R}^{m}, H^{k}\right)}+\left\|\left(\beta, \beta^{\prime}\right)-\left(\gamma, \gamma^{\prime}\right)\right\|_{Z, \alpha ; H^{k}}\right)|t-s|^{2 \alpha} .
\end{aligned}
$$

The third term in (57) is

$$
\begin{aligned}
& {\left[\nabla_{1} \phi\right]_{s t}^{1, x} Z_{s t}^{\beta}\left(x_{s}\right)-\nabla_{1} \phi\left(x_{s}\right) \beta_{s}^{j}\left(x_{s}\right) Z_{s t}^{j}-\left[\nabla_{1} \phi\right]_{s t}^{1, y} Z_{s t}^{\gamma}\left(y_{s}\right)+\nabla_{1} \phi\left(y_{s}\right) \gamma_{s}^{j}\left(y_{s}\right) Z_{s t}^{j}} \\
& =\left[\nabla_{1}^{2} \phi\right]_{s t}^{2, x} \delta x_{s t} Z_{s t}^{\beta}\left(x_{s}\right)-\left[\nabla_{1}^{2} \phi\right]_{s t}^{2, y} \delta y_{s t} Z_{s t}^{\gamma}\left(y_{s}\right)+\nabla_{1} \phi\left(x_{s}\right)\left(Z_{s t}^{\beta}\right)^{\sharp}\left(x_{s}\right)-\nabla_{1} \phi\left(y_{s}\right)\left(Z_{s t}^{\gamma}\right)^{\sharp}\left(y_{s}\right) \\
& =\left(\left[\nabla_{1}^{2} \phi\right]_{s t}^{2, x}-\left[\nabla_{1}^{2} \phi\right]_{s t}^{2, y}\right) \delta x_{s t} Z_{s t}^{\beta}\left(x_{s}\right)+\left[\nabla_{1}^{2} \phi\right]_{s t}^{2, y}\left(\delta x_{s t}-\delta y_{s t}\right) Z_{s t}^{\beta}\left(x_{s}\right)+\left[\nabla_{1}^{2} \phi\right]_{s t}^{2, y} \delta y_{s t}\left(Z_{s t}^{\beta}\left(x_{s}\right)-Z_{s t}^{\beta}\left(y_{s}\right)\right) \\
& +\left[\nabla_{1}^{2} \phi\right]_{s t}^{2, y} \delta y_{s t}\left(Z_{s t}^{\beta}\left(y_{s}\right)-Z_{s t}^{\gamma}\left(y_{s}\right)\right)+\nabla_{1} \phi\left(x_{s}\right)\left(Z_{s t}^{\beta}\right)^{\sharp}\left(x_{s}\right)-\nabla_{1} \phi\left(y_{s}\right)\left(Z_{s t}^{\gamma}\right)^{\sharp}\left(y_{s}\right) .
\end{aligned}
$$


We estimate the first term in the right hand side using Lemma 4.5,

$$
\begin{aligned}
& \left\|E\left[\left(\left[\nabla_{1}^{2} \phi\right]_{s t}^{2, x}-\left[\nabla_{1}^{2} \phi\right]_{s t}^{2, y}\right) \delta x_{s t} Z_{s t}^{\beta}\left(x_{s}\right)\right]\right\|_{H^{k}} \leq\|\phi\|_{C_{b}^{3} \otimes H^{k}}\|x-y\|_{L_{\omega}^{2} L_{t}^{\infty}}\left\|\delta x_{s t}\right\|_{L_{\omega}^{4}}\left\|Z_{s t}^{\beta}\left(x_{s}\right)\right\|_{L_{\omega}^{4}} \\
& \quad \leq C(T)\|\phi\|_{C_{b}^{3} \otimes H^{k}}\|x-y\|_{L_{\omega}^{2} L_{t}^{\infty}}\left\|[x]_{\alpha}\right\|_{L_{\omega}^{4}}\left\|\left(\beta, \beta^{\prime}\right)\right\|_{Z, \alpha ; H^{k}}[\mathbf{Z}]_{\bar{\alpha}}|t-s|^{2 \alpha} .
\end{aligned}
$$

Similarly, using Lemma 4.5 and 4.6 ,

$$
\begin{gathered}
\left\|E\left[\left[\nabla_{1}^{2} \phi\right]_{s t}^{2, y}\left(\delta x_{s t}-\delta y_{s t}\right) Z_{s t}^{\beta}\left(x_{s}\right)\right]\right\|_{H^{k}} \leq C(T)\|\phi\|_{C_{b}^{3} \otimes H^{k}}\left\|[x-y]_{\alpha}\right\|_{L_{\omega}^{2}}\left\|\left(\beta, \beta^{\prime}\right)\right\|_{Z, \alpha ; H^{k}}[\mathbf{Z}]_{\bar{\alpha}}|t-s|^{2 \alpha}, \\
\left\|E\left[\left[\nabla_{1}^{2} \phi\right]_{s t}^{2, y} \delta y_{s t}\left(Z_{s t}^{\beta}\left(x_{s}\right)-Z_{s t}^{\beta}\left(y_{s}\right)\right)\right]\right\|_{H^{k}} \leq C(T)\|\phi\|_{C_{b}^{3} \otimes H^{k}}\left\|[y]_{\alpha}\right\|_{L_{\omega}^{4}}\|x-y\|_{L_{\omega}^{2} L_{t}^{\infty}}\left\|\left(\beta, \beta^{\prime}\right)\right\|_{Z, \alpha ; H^{k}}[\mathbf{Z}]_{\bar{\alpha}}|t-s|^{2 \alpha}, \\
\left\|E\left[\left[\nabla_{1}^{2} \phi\right]_{s t}^{2, y} \delta y_{s t}\left(Z_{s t}^{\beta}\left(y_{s}\right)-Z_{s t}^{\gamma}\left(y_{s}\right)\right)\right]\right\|_{H^{k}} \leq M C(T)\|\phi\|_{C_{b}^{3} \otimes H^{k}}\left\|[y]_{\alpha}\right\|_{L_{\omega}^{1}}\left(\left\|\left(\beta, \beta^{\prime}\right)-\left(\gamma, \gamma^{\prime}\right)\right\|_{Z, \alpha ; H^{k}}\right)[\mathbf{Z}]_{\bar{\alpha}}|t-s|^{2 \alpha} .
\end{gathered}
$$

We estimate the last term in (58) using equation (44) and Lemma4.6,

$$
\begin{aligned}
\left\|E\left[\nabla_{1} \phi\left(x_{s}\right)\left(Z_{s t}^{\beta}\right)^{\sharp}\left(x_{s}\right)-\nabla_{1} \phi\left(y_{s}\right)\left(Z_{s t}^{\gamma}\right)^{\sharp}\left(y_{s}\right)\right]\right\|_{H^{k}} \leq C M\|\phi\|_{C_{b}^{3} \otimes H^{k}} C(T)[\mathbf{Z}]_{\bar{\alpha}} \\
\cdot\left(\|x-y\|_{L_{\omega}^{2} L_{t}^{\infty}}+\left\|\left(\beta, \beta^{\prime}\right)-\left(\gamma, \gamma^{\prime}\right)\right\|_{Z, \alpha ; H^{k}}\right)|t-s|^{2 \alpha} .
\end{aligned}
$$

Thus, there exists $\rho \geq 1$ (which may increase from a line to the next) such that the remainder satisfies, for all $s, t \in[0, T]$,

$$
\begin{aligned}
\| E\left[\phi(x)_{s t}^{\sharp}-\right. & \left.\phi(y)_{s t}^{\sharp}\right]\left\|_{H^{k}} \leq M^{\rho} C(T)\right\| \phi \|_{C_{b}^{3} \otimes H^{k}}|t-s|^{2 \alpha}\left(1+\left\|[x]_{\alpha}\right\|_{L_{\omega}^{4}}+\left\|[y]_{\alpha}\right\|_{L_{\omega}^{4}}\right) \\
& \cdot\left(\|x-y\|_{L_{\omega}^{2} L_{t}^{\infty}}+\left\|[x-y]_{\alpha}\right\|_{L_{\omega}^{2}}+\|\sigma-\theta\|_{L_{t}^{\infty} \mathcal{L}\left(\mathbb{R}^{d} ; H^{k}\right)}+\left\|\left(\beta, \beta^{\prime}\right)-\left(\gamma, \gamma^{\prime}\right)\right\|_{Z, \alpha ; H^{k}}\right) \\
\leq & M^{\rho} C(T)\|\phi\|_{C_{b}^{3} \otimes H^{k}}|t-s|^{2 \alpha}\left(\left\|[x-y]_{\alpha}\right\|_{L_{\omega}^{2}}+\|\sigma-\theta\|_{L_{t}^{\infty} \mathcal{L}\left(\mathbb{R}^{d} ; H^{k}\right)}+\left\|\left(\beta, \beta^{\prime}\right)-\left(\gamma, \gamma^{\prime}\right)\right\|_{Z, \alpha ; H^{k}}\right) .
\end{aligned}
$$

In the last inequality we used Lemma 3.8 combined with Lemma 4.8, and also $\|x\|_{L_{t}^{\infty}} \leq T^{\alpha}[x]_{\alpha}+\left|x_{0}\right|$. We check now the Gubinelli derivative, for each $j$ we have

$$
\begin{aligned}
& \left.\delta\left(\nabla_{1} \phi(x) \beta^{j}(x)\right)_{s t}-\delta\left(\nabla_{1} \phi(y)\right) \gamma^{j}(y)\right)_{s t}=\left[\nabla_{1}^{2} \phi\right]_{s t}^{1 x} \delta x_{s t} \beta_{t}^{j}\left(x_{t}\right)-\left[\nabla_{1}^{2} \phi\right]_{s t}^{1 y} \delta y_{s t} \gamma_{t}^{j}\left(y_{t}\right) \\
& \quad+\nabla_{1} \phi\left(x_{s}\right) \beta_{s t}^{j}\left(x_{s}\right)-\nabla_{1} \phi\left(y_{s}\right) \gamma_{s t}^{j}\left(y_{s}\right)+\nabla_{1} \phi\left(x_{s}\right) \delta\left(\beta_{s t}^{j}(x .)\right)_{s t}-\nabla_{1} \phi\left(y_{s}\right) \delta\left(\gamma_{s t}^{j}(y .)\right)_{s t} .
\end{aligned}
$$

Similarly as for the remainder, we obtain the following,

$$
\begin{aligned}
& \left\|E\left[\delta\left(\nabla_{1} \phi(x) \beta^{j}(x)\right)_{s t}-\delta\left(\left(\nabla_{1} \phi(y)\right) \gamma^{j}(y)\right)_{s t}\right]\right\|_{H^{k}} \\
& \quad \leq M^{\rho} C(T)\|\phi\|_{C_{b}^{3} \otimes H^{k}}|t-s|^{\alpha}\left(\left\|[x-y]_{\alpha}\right\|_{L_{\omega}^{2}}+\|\sigma-\theta\|_{L_{t}^{\infty} \mathcal{L}\left(\mathbb{R}^{d} ; H^{k}\right)}+\left\|\left(\beta, \beta^{\prime}\right)-\left(\gamma, \gamma^{\prime}\right)\right\|_{Z, \alpha ; H^{k}}\right) .
\end{aligned}
$$

We conclude by using Lemma 4.18 to estimate $\left\|[x-y]_{\alpha}\right\|_{L_{\omega}^{2}}$.

\section{Linear Rough PDE}

Let $d, m \in \mathbb{N}$ be fixed and let $\mathbf{Z} \in \mathscr{C}_{g}^{\bar{\alpha}}\left([0, T], \mathbb{R}^{m}\right)$, for $\bar{\alpha} \in\left(\frac{1}{3}, \frac{1}{2}\right)$. Let $\sigma$ and $\beta$ satisfy Assumptions 4.2, for $k$ large enough. In this section we prove well-posedness of measure-valued solutions to linear rough partial differential equations, which are formally given as

$$
\partial_{t} v_{t}=\frac{1}{2} \operatorname{Tr} \nabla^{2}\left(\sigma_{t} \sigma_{t}^{T} v_{t}\right)-\operatorname{div}\left(\beta_{t} \dot{Z}_{t} v_{t}\right), \quad v_{0} \in \mathcal{P}\left(\mathbb{R}^{d}\right)
$$

To rigorously define the meaning of a solution to equation (59), we take a slightly more general approach, as described below. 
Assumptions 5.1. Let $n \in \mathbb{N}$ and $\alpha \in\left(\frac{1}{3}, \bar{\alpha}\right)$.

(i) Let a : $[0, T] \rightarrow C^{n+3}\left(\mathbb{R}^{d} ; \mathbb{R}^{d \times d}\right)$ be a measurable path such that $a_{t}^{i, j}(x) \xi^{i} \xi^{j} \geq 0$ for all $x, \xi \in \mathbb{R}^{d}$ and $t \in[0, T]$.

(ii) Let $\mathbf{X} \in \mathscr{C}_{g}^{\alpha}\left([0, T] ; C_{b}^{n+3}\left(\mathbb{R}^{d} ; \mathbb{R}^{d}\right)\right)$ be a geometric rough path, as described in Section 2

The examples we have in mind are $a=\frac{1}{2} \sigma \sigma^{T}$ and $\mathbf{X}=\int \beta_{r} d \mathbf{Z}_{r}$, as described in Proposition 5.5. In order to describe the main ideas, we argue now on a formal level assuming smoothness in time of $X$; rigorous definitions in the rough path case will be given later in the section. We study uniqueness of solutions to the following linear equation

$$
\partial_{t} v_{t}=\operatorname{Tr} \nabla^{2}\left(a_{t} v_{t}\right)+\operatorname{div}\left(\dot{X}_{t} v_{t}\right), \quad v_{0} \in \mathcal{P}\left(\mathbb{R}^{d}\right) .
$$

The proof is based on a backward duality trick; suppose we can show existence of a sufficiently regular solution to the backward PDE

$$
\partial_{t} u_{t}+\operatorname{Tr}\left(a_{t} \nabla^{2} u_{t}\right)=\dot{X}_{t} \nabla u_{t}
$$

for a given final condition $u_{T}$, then at least formally we have

$$
\partial_{t} v_{t}\left(u_{t}\right)=\left(\operatorname{Tr} \nabla^{2}\left(a_{t} v_{t}\right)\left(u_{t}\right)-v_{t}\left(\operatorname{Tr}\left(a_{t} \nabla^{2} u_{t}\right)\right)+\operatorname{div}\left(\dot{X}_{t} v_{t}\right)\left(u_{t}\right)+v_{t}\left(\dot{X}_{t} \nabla u_{t}\right)=0,\right.
$$

which shows that $v_{T}\left(u_{T}\right)=v_{0}\left(u_{0}\right)$. Now, if $u_{T}$ is chosen in a class of functions large enough to fully determine $v_{T}$, we see that it will be fully determined by $v_{0}$ and $u_{0}$, thus showing uniqueness.

For simplicity only, we write equation (61) on divergence form and as a forward equation as follows

$$
\partial_{t} u_{t}=\operatorname{div}\left(a_{t} \nabla u_{t}\right)+\dot{X}_{t} \nabla u_{t}, \quad u_{0} \text { given, }
$$

which can be seen to be equivalent to (61) by replacing $X_{t}$ by $\left(\int_{0}^{t} \nabla a_{r} d r, X_{t}\right)$ in (63) and then reversing time, i.e. $u_{t} \mapsto u_{T-t}$.

The strategy to prove existence of a smooth solution to (63) is as follows. We first show how to give an intrinsic notion of solution of (60) and (63) in the context of the so-called unbounded rough drivers, see [2]. We then replace $X$ by smooth vector fields, in which case it is well know that there exists a unique solution of (63) which is smooth provided the coefficients are. We then consider the vector of derivatives $f=\left(u, \nabla u, \ldots, \nabla^{n} u\right)$ and show that $f$ satisfies a vector valued equation, for which we can find bounds independent of $\dot{X}$. The equation for $f$ will be solved in the space $L^{2}\left(\mathbb{R}^{d} ; \mathbb{R}^{N}\right)$, thus giving bounds on $u$ in the Sobolev-space $H^{n}\left(\mathbb{R}^{d}\right)$.

Second, we approximate $\mathbf{X}$ by a sequence of smooth vector fields and show that the corresponding sequence of solutions converge to a meaningful solution of (63). Since the solution is in $H^{n}\left(\mathbb{R}^{d}\right)$ we can use Sobolev embedding [4, Corollary 9.13] to show the needed spatial regularity to justify the computations in (62).

The techniques used to prove the first step are motivated by [2] and [11], and the main technical tool is the a priori estimate found in [11].

\subsection{Unbounded rough drivers}

We start by rephrasing (63) in terms of so called unbounded rough drivers. The main motivation for doing so is the a priori estimate from [11]. 
Assume that $X$ is a smooth path, then equation (63) is well defined as a PDE. Integrating (63) from $s$ to $t$ we obtain

$$
\delta u_{s t}=\int_{s}^{t} \operatorname{div}\left(a_{r} \nabla u_{r}\right) d r+\int_{s}^{t} \dot{X}_{r} \nabla u_{r} d r
$$

Iterating the equation into itself we obtain

$$
\delta u_{s t}=\int_{s}^{t} \operatorname{div}\left(a_{r} \nabla u_{r}\right) d r+B_{s t}^{1} u_{s}+B_{s t}^{2} u_{s}+u_{s t}^{\natural}
$$

where at least formally,

$$
B_{s t}^{1} \phi=X_{s t}^{j} \partial_{j} \phi, \quad B_{s t}^{2} \phi=\int_{s}^{t} \dot{X}_{r}^{j} \partial_{j} \int_{s}^{r} \dot{X}_{u}^{i} \partial_{i} \phi d u d r
$$

and

$$
\begin{aligned}
u_{s t}^{\natural} & =\int_{s}^{t} \dot{X}_{r}^{j} \partial_{j} \int_{s}^{r} \dot{X}_{\tau}^{i} \partial_{i} \int_{s}^{\tau} \dot{X}_{\theta}^{l} \partial_{l} u_{\theta} d \theta d \tau d r \\
& +\int_{s}^{t} \dot{X}_{r}^{j} \partial_{j} \int_{s}^{r} \operatorname{div}\left(a_{\tau} \nabla u_{\tau}\right) d \tau d r+\int_{s}^{t} \dot{X}_{r}^{j} \partial_{j} \int_{s}^{r} \dot{X}_{\tau}^{i} \partial_{i} \int_{s}^{\tau} \operatorname{div}\left(a_{\theta} \nabla u_{\theta}\right) d \theta d \tau d r .
\end{aligned}
$$

By the usual power counting the remainder term $u^{\natural}$ should be regular in time, but we notice that in general it is a distribution in space. Following [2] we call a scale of spaces a quadruple $\left(E^{n}\right)_{n=0}^{3}$ of Banach spaces such that $E^{n+1}$ is continuously embedded into $E^{n}$. Let $E^{-n}$ be the topological dual of $E^{n}$ (in general, $E^{-0} \neq E^{0}$ ).

Definition 5.2. An unbounded $\alpha$-rough driver on the scale $\left(E^{n}\right)_{n}$, is a pair $\mathbf{B}=\left(B^{1}, B^{2}\right)$ of mappings on $E^{n}$ such that

$$
\left\|B_{s t}^{1}\right\|_{\mathcal{L}\left(E^{n}, E^{n-1}\right)} \lesssim|t-s|^{\alpha} \text { for }-2 \leq n \leq 3, \quad\left\|B_{s t}^{2}\right\|_{\mathcal{L}\left(E^{n}, E^{n-2}\right)} \lesssim|t-s|^{2 \alpha} \text { for } 0 \leq n \leq 2,
$$

and Chen's relation is satisfied,

$$
\delta B_{s r t}^{1}=0, \quad \delta B_{s r t}^{2}=B_{r t}^{1} B_{s r}^{1}, \quad \forall s<r<t .
$$

We shall write $\|\mathbf{B}\|_{\alpha}$ for the smallest constant dominating the bounds in (66).

We show how to construct an unbounded rough driver given a rough path.

Proposition 5.3. Let $N \in \mathbb{N}$ and $\mathbf{X}$ satisfy Assumption 4.2|(ii), Define for $\phi \in C_{c}^{\infty}\left(\mathbb{R}^{d} ; \mathbb{R}^{N}\right)$

$$
B_{s t}^{1} \phi(x)=X_{s t}^{j}(x) \partial_{j} \phi(x), \quad B_{s t}^{2} \phi(x)=\left(\nabla_{1}^{\otimes} \mathbb{X}_{s t}\right)^{j}(x, x) \partial_{j} \phi(x)+\mathbb{X}_{s t}^{i, j}(x, x) \partial_{i} \partial_{j} \phi(x)
$$

where $\nabla_{1}^{\otimes}: C_{b}^{3}\left(\mathbb{R}^{d} \times \mathbb{R}^{d} ; \mathbb{R}^{d \times d}\right) \rightarrow C_{b}^{2}\left(\mathbb{R}^{d} \times \mathbb{R}^{d} ; \mathbb{R}^{d}\right)$ is the linear extension of the map defined on the algebraic tensor as

$$
\nabla_{1}^{\otimes}(f \otimes g)^{j}(x, y)=g^{i}(y) \partial_{i} f^{j}(x) .
$$

Then $\mathbf{B}:=\left(B^{1}, B^{2}\right)$ is an unbounded rough driver on both scales $E_{n}:=W^{n, \rho}\left(\mathbb{R}^{d} ; \mathbb{R}^{N}\right), \rho \geq 1$, and $E_{n}:=C_{b}^{n}\left(\mathbb{R}^{d} ; \mathbb{R}^{N}\right)$. Moreover, the mapping $\mathbf{X} \mapsto \mathbf{B}$ is continuous in the operator norm. 
Proof. Let $0 \leq s \leq \theta \leq t$. By Chen's relation for rough paths (10), and (68)

$$
\delta\left[\left(\nabla_{1}^{\otimes} \mathbb{X}\right)^{j}(x, x) \partial_{j} \phi(x)\right]_{s \theta t}=\nabla_{1}^{\otimes}\left(X_{s \theta} \otimes X_{\theta t}\right)^{j}(x, x) \partial_{j} \phi(x)=X_{\theta t}^{i}(x) \partial_{i} X_{s \theta}^{j}(x) \partial_{j} \phi(x)
$$

which gives

$$
\delta B_{s \theta t}^{2} \phi(x)=X_{\theta t}^{i}(x) \partial_{i} X_{s \theta}^{j}(x) \partial_{j} \phi(x)+X_{s \theta}^{i}(x) X_{\theta t}^{j}(x) \partial_{i} \partial_{j} \phi(x)=X_{\theta t}^{i}(x) \partial_{i}\left[X_{s \theta}^{j}(x) \partial_{j} \phi(x)\right] .
$$

Continuity of the mapping follows immediately from the continuity of $\nabla_{x}^{\otimes}$.

We notice that there is no zero order term in the above unbounded rough driver. We include such a term by considering a rough path $\mathbf{X} \in \mathscr{C}^{\alpha}\left([0, T] ; C_{b}^{3}\left(\mathbb{R}^{1+d} ; \mathbb{R}^{1+d}\right)\right)$, i.e. with an additional spatial variable. Then, for $\phi \in C_{c}^{\infty}\left(\mathbb{R}^{d} ; \mathbb{R}^{N}\right)$ let

$$
\begin{aligned}
B_{s t}^{1} \phi(x)= & X_{s t}^{j}(x) \partial_{j} \phi(x)+X_{s t}^{0} \phi(x) \\
B_{s t}^{2} \phi(x)= & \left(\nabla_{1}^{\otimes} \mathbb{X}_{s t}\right)^{j}(x, x) \partial_{j} \phi(x)+\mathbb{X}_{s t}^{i, j}(x, x) \partial_{i} \partial_{j} \phi(x) \\
& +\mathbb{X}_{s t}^{0,0}(x, x) \phi(x)+\mathbb{X}_{s t}^{0, j}(x, x) \partial_{j} \phi(x)+\left(\nabla_{1}^{\otimes} \mathbb{X}_{s t}\right)^{0}(x, x) \phi(x),
\end{aligned}
$$

where we make the convention that summation over repeated indexes are over $1 \leq j \leq d$, i.e. excluding 0 .

With this in hand we can define the notion of a solution of (60).

Definition 5.4. A path $v:[0, T] \rightarrow \mathcal{M}\left(\mathbb{R}^{d}\right) \subset\left(C_{b}\left(\mathbb{R}^{d}\right)\right)^{*}$ is a solution to 60 if for all $\phi \in C_{b}^{3}\left(\mathbb{R}^{d}\right)$ the mapping defined by

$$
v_{s t}^{\natural}(\phi):=\delta v_{s t}(\phi)-\int_{s}^{t} v_{r}\left(\operatorname{Tr}\left(a_{r} \nabla^{2} \phi\right)\right) d r-v_{s}\left(B_{s t}^{1} \phi\right)-v_{s}\left(B_{s t}^{2} \phi\right)
$$

satisfies $\left|v_{s t}^{\natural}(\phi)\right| \lesssim|t-s|^{3 \alpha}\|\phi\|_{C_{b}^{3}}$. Above $\mathbf{B}=\left(B^{1}, B^{2}\right)$ is the unbounded rough driver constructed from $\mathbf{X}$ as in Proposition 5.3

We see now that, in the special case when $a=\frac{1}{2} \sigma \sigma^{T}$ and $\mathbf{X}=\int \beta_{r} d \mathbf{Z}_{r}$, existence of solutions follows from the results of Sections 3 and 4.

Proposition 5.5. Let $\rho \geq 2$ and let $\left(\Omega, \mathcal{F},\left(\mathcal{F}_{t}\right)_{t \in[0, T]}, P\right)$ be a probability space that supports a $d$ dimensional Brownian motion $W$ and an $\mathcal{F}_{0}$-measurable random variable, $\Xi \in L^{\rho}\left(\Omega ; \mathbb{R}^{d}\right)$ such that the push-forward measure $P_{*}(\Xi)=v_{0}$. Let $\mathbf{Z} \in \mathscr{C}_{\text {wg }}^{\bar{\alpha}}\left([0, T] ; \mathbb{R}^{m}\right)$ be a a weakly geometric rough path. Under Assumption 4.2 we have

(i) B, generated by the rough path $\int \beta_{r} d \mathbf{Z}_{r}$ as in Proposition 5.3 is an unbounded rough driver as in Definition 5.2

(ii) There exists a solution $v$ of (60) driven by $\mathbf{B}$, in the sense of Definition 5.4 This solution is given by $v_{t}=\mathcal{L}\left(x_{t}\right)$, where, for $P$-a.e. $\omega \in \Omega, x(\omega)$ is the unique solution to equation (20) with initial condition $\Xi(\omega)$, driven by the random rough driver $\mathbf{F}$ constructed in Lemma 4.8

Proof. From Sobolev embedding theorem [4, Corollary 9.13], we have $\beta \in \mathscr{D}_{Z}^{2 \alpha}\left([0, T] ; C_{b}^{3}\left(\mathbb{R}^{d} ; \mathbb{R}^{d}\right)\right)$. Thus, using the construction (11), we have that $\int \beta_{r} d \mathbf{Z}_{r}$ is a rough path over $C_{b}^{3}\left(\mathbb{R}^{d} ; \mathbb{R}^{d}\right)$. The first claim follows now by Proposition 5.3. 
We prove now the second claim. It follows from Proposition 4.13 that the stochastic process $\left(x_{t}\right)_{t \in[0, T]}$ is adapted. We can thus define $v:=\mathcal{L}(x)$ and denote by $v_{t}$ the induced time-marginals. From Itô's formula, Proposition 4.14, we get

$$
v_{t}(\phi)=v_{0}(\phi)+\int_{0}^{t} \frac{1}{2} v_{r}\left(\operatorname{Tr}\left(\nabla^{2} \phi \sigma_{r} \sigma_{r}^{T}\right)\right) d r+\int_{0}^{t} v_{r}\left(\nabla \phi \beta_{r}\right) d \mathbf{Z}_{r}
$$

The proof is complete once we show that $\int_{0}^{t} v_{r}\left(\nabla \phi \beta_{r}\right) d \mathbf{Z}_{r}$ has an expansion in terms of the unbounded rough driver. Recall that we get from Lemma 4.15, we have

$$
\left(v(\nabla \phi \beta), v\left(\nabla^{2} \phi(\beta \otimes \beta)+\nabla \phi\left(\nabla \beta \beta+\beta^{\prime}\right)\right) \in \mathscr{D}_{Z}^{2 \alpha}\left([0, T] ; H^{k}\right)\right.
$$

and this gives, using the sewing lemma 2.1 ,

$$
\mid \int_{s}^{t} v_{r}\left(\phi \beta_{r}\right) d \mathbf{Z}_{r}-v_{s}\left(\nabla \phi \beta_{s}^{j}\right) Z_{s t}^{j}-v_{s}\left(\nabla^{2} \phi \beta_{s}^{j} \otimes \beta_{s}^{i}+\nabla \phi\left(\nabla \beta_{s}^{j} \beta_{s}^{i}+\beta_{s}^{j, i}\right) \mathbb{Z}_{s t}^{i, j} \mid \lesssim\|\phi\|_{C_{b}^{3}|t-s|^{3 \alpha}} .\right.
$$

Regrouping the terms we can write

$$
\left|\int_{s}^{t} v_{r}\left(\phi \beta_{r}\right) d \mathbf{Z}_{r}-v_{s}\left(\beta_{s}^{j} \nabla \phi Z_{s t}^{j}+\beta_{s}^{j, i} \nabla \phi \mathbb{Z}_{s t}^{i, j}\right)-v_{s}\left(\beta_{s}^{j} \nabla\left(\beta_{s}^{i} \nabla \phi\right) \mathbb{Z}_{s t}^{i, j}\right)\right| \lesssim\|\phi\|_{C_{b}^{3}}|t-s|^{3 \alpha} .
$$

By definition of $B^{1}$ we get

$$
\left\|B_{s t}^{1} \phi-\beta_{s}^{j} \nabla \phi Z_{s t}^{j}-\beta_{s}^{j, i} \nabla \phi \mathbb{Z}_{s t}^{i, j}\right\|_{C_{b}} \lesssim\|\phi\|_{C_{b}^{1}}|t-s|^{3 \alpha},
$$

which gives

$$
\left|v_{s}\left(\beta_{s}^{j} \nabla \phi Z_{s t}^{j}+\beta_{s}^{j, i} \nabla \phi \mathbb{Z}_{s t}^{i, j}\right)-v_{s}\left(B_{s t}^{1} \phi\right)\right| \lesssim\|\phi\|_{C_{b}^{1}}|t-s|^{3 \alpha} .
$$

Moreover

$$
\begin{aligned}
\left\|B_{s t}^{2} \phi-\beta_{s}^{j} \nabla\left(\beta_{s}^{i} \nabla \phi\right) \mathbb{Z}_{s t}^{i, j}\right\|_{C_{b}} \lesssim \| \beta_{s}^{j} \nabla B_{s}^{1} \phi Z_{s t}^{j}+\beta_{s}^{j, i} \nabla B_{s}^{1} \phi \mathbb{Z}_{s t}^{i, j}+\beta_{s}^{j} \nabla\left(\beta_{s}^{i} \nabla\right) \phi \mathbb{Z}_{s t}^{i, j} \\
\quad-\left(\beta_{s}^{j} \nabla Z_{s t}^{j}+\beta_{s}^{j, i} \nabla \mathbb{Z}_{s t}^{i, j}\right) B_{s}^{1} \phi-\beta_{s}^{j} \nabla\left(\beta_{s}^{i} \nabla \phi\right) \mathbb{Z}_{s t}^{i, j}\left\|_{C_{b}}+\right\| \phi \|_{C_{b}^{2}}|t-s|^{3 \alpha} \\
\quad=\|\phi\|_{C_{b}^{2}|t-s|^{3 \alpha} .}
\end{aligned}
$$

This shows that we may rewrite the equation for $v$ as

$$
\delta v_{s t}(\phi)=\int_{s}^{t} \frac{1}{2} v_{r}\left(\operatorname{Tr}\left(\nabla^{2} \phi \sigma_{r} \sigma_{r}^{T}\right)\right) d r+v_{s}\left(B_{s t}^{1} \phi+B_{s t}^{2} \phi\right)+v_{s t}^{\natural}(\phi)
$$

where $v^{\natural} \in C_{2}^{3 \alpha}\left([0, T] ;\left(C_{b}^{3}\left(\mathbb{R}^{d}\right)\right)^{*}\right)$ is a remainder.

\subsection{A priori estimates for smooth vector fields}

For this section we consider an approximation of equation (64), driven by a smooth (in time) driver,

$$
\partial_{t} u=\operatorname{div}(a \nabla u)+\dot{X} \nabla u
$$

where $X$ is smooth. We will find bounds on $u$ in $H^{n}\left(\mathbb{R}^{d}\right)$ depending only on a canonical unbounded rough driver generated by $X$. The first step towards this goal is to write $u$ and all the derivatives as a vector in an $L^{2}$ space. 
Let $u$ denote the (smooth) solution of (70) and let $f=\left(u, \nabla u, \ldots, \nabla^{n} u\right)$ denote the vector of gradients as taking values in the truncated tensor algebra $T^{(n)}\left(\mathbb{R}^{d}\right)=\bigoplus_{q=0}^{n}\left(\mathbb{R}^{d}\right)^{\otimes q}$. We will simply write $g v$ for the 1-contractive product

$$
\left(\mathbb{R}^{d}\right)^{\otimes q} \times\left(\mathbb{R}^{d}\right)^{\otimes r} \rightarrow\left(\mathbb{R}^{d}\right)^{\otimes(q+r-2)},
$$

e.g. for a $g \in\left(\mathbb{R}^{d}\right)^{\otimes 2}$ and $v \in \mathbb{R}^{d}$ the product $g v$ has component $i$ given by $g_{i j} v_{j}$.

Using Leibniz formula we have

$$
\nabla^{q}(\dot{X} \nabla u)=\sum_{j=0}^{q}\left(\begin{array}{c}
q \\
j
\end{array}\right) \nabla^{q-j} \dot{X} \nabla^{j+1} u=\sum_{j=0}^{q-1}\left(\begin{array}{c}
q \\
j
\end{array}\right) \nabla^{q-j} \dot{X} f^{(j+1)}+\dot{X} \nabla f^{(q)}=: \dot{X} \nabla f^{(q)}+M_{\dot{X}}^{(q)} f
$$

where $M_{\dot{X}}^{(q)}: T^{(n)}\left(\mathbb{R}^{d}\right) \rightarrow\left(\mathbb{R}^{d}\right)^{\otimes q}$ is given by

$$
M_{\dot{X}}^{(q)}\left(\bigoplus_{j=0}^{n} y^{(j)}\right)=\sum_{j=0}^{q-1}\left(\begin{array}{c}
q \\
j
\end{array}\right) \nabla^{q-j} \dot{X} y^{(j+1)} .
$$

We notice that the above sum is in $\left(\mathbb{R}^{d}\right)^{\otimes q}$ since we are doing a contractive product of $\left(\mathbb{R}^{d}\right)^{\otimes(q-j+1)}$ and $\left(\mathbb{R}^{d}\right)^{\otimes(j+1)}$.

For each $q$ we have

$$
\begin{aligned}
\partial_{t} f^{(q)} & =\nabla^{q} \operatorname{div}(a \nabla u)+\nabla^{q}(\dot{X} \nabla u)=\operatorname{div}\left(a \nabla f^{(q)}\right)+\nabla\left(M_{a}^{(q)} f\right)+\left(\dot{X} \nabla f^{(q)}+M_{\dot{X}}^{(q)} f\right) \\
& =\operatorname{div}\left(a \nabla f^{(q)}\right)+M_{\nabla a}^{(q)} f+M_{a}^{(q)} \nabla f+\left(\dot{X} \nabla f^{(q)}+M_{\dot{X}}^{(q)} f\right) .
\end{aligned}
$$

This gives that $f$ satisfies the $T^{(n)}\left(\mathbb{R}^{d}\right)$-valued equation

$$
\partial_{t} f=\operatorname{div}(a \nabla f)+\dot{V} \nabla f+\dot{Y} f
$$

where we have set

$$
\dot{V}=\bigoplus_{q=0}^{n}\left(M_{a}^{(q)}+\dot{X}\right), \quad \dot{Y}=\bigoplus_{q=0}^{n}\left(M_{\nabla a}^{(q)}+M_{\dot{X}}^{(q)}\right) .
$$

Remark 5.6. We notice that if we replace $X$ above by $X^{\epsilon}$ where $\mathbf{X}^{\epsilon}$ converges to a rough path $\mathbf{X}$, then the corresponding coefficients $V^{\epsilon}, Y^{\epsilon}$ have canonical rough path lifts, $\mathbf{V}^{\epsilon}$ and $\mathbf{Y}^{\epsilon}$, with values in $C_{b}^{3}$ which remain bounded uniformly in $\epsilon$. This comes from the fact that there are canonical iterated integrals between the $C_{b}^{3}$-valued paths $t \mapsto \int_{0}^{\cdot} a_{r}(x) d r$ and $t \mapsto X_{t}(x)$,

$$
\int_{s}^{t} X_{s r}(x) a_{r}(y) d r, \quad \int_{s}^{t} a_{s r}(x) d X_{r}(y)
$$

where the first term is simply the Riemann-integral and the second term is defined using integration by parts as before.

Given the previous construction, we consider now a system of equations. We remark that this is not just a vector valued version of the results found in [18], since we are not interested in energy estimates. Indeed, the matrix $a$ is allowed to be degenerate but we require spatial smoothness. We consider the equation

$$
\partial_{t} f=\operatorname{div}(a \nabla f)+\dot{V} \nabla f+\dot{Y} f
$$


for given functions $a$ and $\dot{V}, \dot{Y}$ smooth in time, and a given initial condition $f_{0}$. The solution is a vector valued function $f:[0, T] \times \mathbb{R}^{d} \rightarrow \mathbb{R}^{N}$, and the coefficients are on the form

$$
\begin{aligned}
& \dot{Y}:[0, T] \times \mathbb{R}^{d} \rightarrow \mathbb{R}^{N} \otimes \mathbb{R}^{N}, \quad \dot{V}:[0, T] \times \mathbb{R}^{d} \rightarrow \mathcal{L}\left(\mathbb{R}^{d} \otimes \mathbb{R}^{N} ; \mathbb{R}^{N}\right), \\
& a:[0, T] \times \mathbb{R}^{d} \rightarrow \mathbb{R}^{d} \otimes \mathbb{R}^{d} .
\end{aligned}
$$

We will assume that $a$ is diagonal in (73), so component $l$ reads

$$
\partial_{t} f^{l}=\partial_{i}\left(a^{i, j} \partial_{j} f^{l}\right)+\dot{V}_{i}^{l, m} \partial_{i} f^{m}+\dot{Y}^{l, m} f^{m}, \quad 1 \leq l \leq N .
$$

We begin with our main a priori estimate.

Proposition 5.7. Assume $f$ is a solution of (73). Then there exists a constant $C=C\left(a, B^{1}, B^{2}\right)$ such that

$$
\sup _{t \in[0, T]}\left\|f_{t}\right\|_{L^{2}\left(\mathbb{R}^{d} ; \mathbb{R}^{N}\right)} \leq C\left\|f_{0}\right\|_{L^{2}\left(\mathbb{R}^{d}\right)}, \quad\left\|\delta f_{s t}\right\|_{H^{-1}\left(\mathbb{R}^{d} ; \mathbb{R}^{N}\right)} \leq C|t-s|^{\alpha}
$$

where $\left(B^{1}, B^{2}\right)$ is an unbounded rough driver depending only on the rough path lift of the path $(V, Y)$.

Proof. The finite-dimensional tensor $\left(f^{\otimes 2}\right)^{n, l}:=f^{n} f^{l}$ then satisfies

$$
\begin{aligned}
\partial_{t}\left(f^{\otimes 2}\right) & =2 f \hat{\otimes} \partial_{t} f=2 f \hat{\otimes} \operatorname{div}(a \nabla f)+2 f \hat{\otimes} \dot{V} \nabla f+2 f \hat{\otimes} \dot{Y} f \\
& =2 f \hat{\otimes} \operatorname{div}(a \nabla f)+\dot{\bar{V}} \nabla f^{\otimes 2}+\dot{\bar{Y}} f^{\otimes 2}
\end{aligned}
$$

where

$$
\bar{V}:=2 \operatorname{Id} \hat{\otimes} V:=\operatorname{Id} \otimes V+V \otimes \operatorname{Id}, \quad \bar{Y}:=2 \operatorname{Id} \hat{\otimes} Y:=\operatorname{Id} \otimes Y+Y \otimes \operatorname{Id},
$$

both belongs to the space $\mathcal{L}\left(\mathbb{R}^{N} \otimes \mathbb{R}^{N} ; \mathbb{R}^{N} \otimes \mathbb{R}^{N}\right)$. Define now the unbounded rough driver

$$
B_{s t}^{\otimes 2,1} \phi=\int_{s}^{t} \dot{\bar{V}}_{r} \nabla \phi+\dot{\bar{Y}}_{r} \phi d r, \quad B_{s t}^{\otimes 2,2} \phi=\int_{s}^{t}\left[\dot{\bar{V}}_{r} \nabla \cdot+\dot{\bar{Y}}_{r}\right] \int_{s}^{r} \dot{\bar{V}}_{\theta} \nabla \phi+\dot{\bar{Y}}_{\theta} \phi d \theta d r
$$

and the drift

$$
m_{t}^{\otimes 2}(\phi)=-\int_{s}^{t} 2\left(a \nabla f^{n}, \nabla f^{l} \phi^{l, n}\right)-\left(f^{n} f^{l}, \nabla\left(a \nabla \phi^{l, n}\right)\right) d r
$$

for functions $\phi: \mathbb{R}^{d} \rightarrow \mathbb{R}^{N} \otimes \mathbb{R}^{N}$. This gives the dynamics

$$
\delta f_{s t}^{\otimes 2}=\delta m_{s t}^{\otimes 2}+B_{s t}^{\otimes 2,1} f_{s}^{\otimes 2}+B_{s t}^{\otimes 2,2} f_{s}^{\otimes 2}+f_{s t}^{\otimes 2, \natural}
$$

on the scale $\left(W^{n, \infty}\left(\mathbb{R}^{d} ; \mathbb{R}^{N} \otimes \mathbb{R}^{N}\right)\right)_{n}$. Let $\phi \in W^{2, \infty}\left(\mathbb{R}^{d} ; \mathbb{R}^{N} \otimes \mathbb{R}^{N}\right)$ and write

$$
\left|\delta m_{s t}^{\otimes 2}(\phi)\right| \leq\|\phi\|_{W^{1, \infty}} \int_{s}^{t} 2\left\|\left(\nabla f^{l}\right)^{T} a \nabla f^{n}\right\|_{L^{1}}+\|a\|_{W^{1, \infty}}\|f\|_{L^{2}}^{2} d r
$$

which shows that $m^{\otimes 2}$ has bounded variation in $\left(W^{2, \infty}\left(\mathbb{R}^{d} ; \mathbb{R}^{N} \otimes \mathbb{R}^{N}\right)^{*}\right)$.

Now, by the a priori bounds, [11, Theorem 2.9], we get

$$
\left\|f_{s t}^{\otimes 2, \natural}\right\|_{\left(W^{3, \infty}\right)^{*}} \leq C\left(\|f\|_{L^{\infty}\left(s, t L^{2}\right)}^{2}|t-s|^{3 \alpha}+|t-s|^{\alpha} \int_{s}^{t} 2\left\|\left(\nabla f^{l}\right)^{T} a \nabla f^{n}\right\|_{L^{1}}+\|a\|_{W^{1, \infty}}\|f\|_{L^{2}}^{2} d r\right) .
$$


where $C$ depends on $\left\|\mathbf{B}^{\otimes 2}\right\|_{\alpha}$. Testing $f^{\otimes 2}$ against the $N \times N$ identity matrix $I_{N \times N}$ and using that $a$ is positive semi-definite we get

$$
\begin{aligned}
\delta\left(\|f\|_{L^{2}}^{2}\right)_{s t} & =\delta m_{s t}^{\otimes 2}\left(I_{N \times N}\right)+f_{s}^{\otimes 2}\left(B_{s t}^{\otimes 2,1, *} I_{N \times N}+B_{s t}^{\otimes 2,2, *} I_{N \times N}\right)+f_{s t}^{\otimes 2, \natural}\left(I_{N \times N}\right) \\
& \leq-2 \int_{s}^{t}\left\|\left(\nabla f^{n}\right)^{T} a \nabla f^{n}\right\|_{L^{1}} d r+\left\|f_{s}\right\|_{L^{2}}^{2}\left\|\mathbf{B}^{\otimes 2}\right\|\left\|_{\alpha}|t-s|^{\alpha}+\right\| f_{s}\left\|_{L^{2}}^{2}\right\| \mathbf{B}^{\otimes 2}\left\|_{\alpha}|t-s|^{2 \alpha}+\right\| f_{s t}^{\otimes 2, \natural} \|_{\left(W^{3, \infty}\right)^{*}} \\
& \leq-2 \int_{s}^{t}\left\|\left(\nabla f^{n}\right)^{T} a \nabla f^{n}\right\|_{L^{1}} d r+C\|f\|_{L^{\infty}\left(s, t ; L^{2}\right)}^{2}|t-s|^{\alpha} \\
& +C|t-s|^{\alpha} \int_{s}^{t} 2\left\|\left(\nabla f^{l}\right)^{T} a \nabla f^{n}\right\|_{L^{1}}+\|a\|_{W^{1, \infty}}\|f\|_{L^{2}}^{2} d r
\end{aligned}
$$

Note that $\left\|\left(\nabla f^{l}\right)^{T} a \nabla f^{n}\right\|_{L^{1}} \leq N\left\|\left(\nabla f^{n}\right)^{T} a \nabla f^{n}\right\|_{L^{1}}$. Indeed, write $a=\frac{1}{2} \sigma \sigma^{T}$ and use the CauchySchwarz inequality

$$
\left(\nabla f^{l}\right)^{T} a \nabla f^{n}=\frac{1}{2}\left(\sigma^{T} \nabla f^{l}\right)^{T}\left(\sigma^{T} \nabla f^{n}\right) \leq \frac{1}{2}\left|\sigma^{T} \nabla f^{l} \| \sigma^{T} \nabla f^{n}\right| .
$$

Summing over $l$ and $n$ gives that the above is bounded by $\frac{N}{2} \sum_{n=1}^{N}\left|\sigma^{T} \nabla f^{n}\right|^{2}$. Integrating w.r.t. $x$ we get the claim.

If we choose $s, t$ such that $C N|t-s|^{\alpha} \leq \frac{1}{2}$ we get

$$
\delta\left(\|f\|_{L^{2}}^{2}\right)_{s t} \leq C\|f\|_{L^{\infty}\left(s, t ; L^{2}\right)}^{2}\left(|t-s|^{\alpha}+\|a\|_{W^{1, \infty}}(t-s)\right) .
$$

From the rough Gronwall lemma, [11, Lemma 2.11], the first bound of (75) holds.

For the second inequality we notice that the evolution of $f$ on $W^{n, 2}\left(\mathbb{R}^{d} ; \mathbb{R}^{N}\right)$ reads

$$
\delta f_{s t}=\delta m_{s t}+B_{s t}^{1} f_{s}+B_{s t}^{2} f_{s}+f_{s t}^{\natural}
$$

where $m_{t}=\int_{0}^{t} \operatorname{div}\left(a_{r} \nabla f_{r}\right) d r$ and we have defined the unbounded rough driver

$$
B_{s t}^{1} \phi=\int_{s}^{t} \dot{V}_{r} \nabla \phi+\dot{Y}_{r} \phi d r, \quad B_{s t}^{2} \phi=\int_{s}^{t}\left[\dot{V}_{r} \nabla \cdot+\dot{Y}_{r}\right] \int_{s}^{r} \dot{V}_{\theta} \nabla \phi+\dot{Y}_{\theta} \phi d \theta d r
$$

Since the operator is self-adjoint it is easy to bound the variation of $m$ in $H^{-2}$;

$$
\left|\delta m_{s t}(\phi)\right| \leq(t-s)\|f\|_{L^{\infty}\left([s, t] ; L^{2}\right)}\|a\|_{W^{1, \infty}}\|\phi\|_{H^{2}} \leq(t-s) C\left\|f_{0}\right\|_{L^{2}}\|a\|_{W^{1, \infty}}\|\phi\|_{H^{2}} .
$$

This gives, using [11, Theorem 2.9],

$$
\left\|f_{s t}^{\natural}\right\|_{H^{-3}} \lesssim C|t-s|^{3 \alpha}\left\|f_{0}\right\|_{L^{2}} .
$$

where $C$ depends on $\|\mathbf{B}\|_{\alpha}$ and $\|a\|_{W^{1, \infty}}$. Take now a mollifier $\psi_{\eta}$ and decompose $\phi=\psi_{\eta} * \phi+(I-$ $\left.\psi_{\eta}\right) * \phi$ for any $\eta>0$ and any test function $\phi \in H^{1}\left(\mathbb{R}^{d} ; \mathbb{R}^{N}\right)$. This gives

$$
\left|\left(\delta f_{s t},\left(I-\psi_{\eta}\right) * \phi\right)\right| \lesssim\|f\|_{L^{\infty}\left([s, t] ; L^{2}\right)}\left\|\left(I-\psi_{\eta}\right) * \phi\right\|_{L^{2}} \lesssim\left\|f_{0}\right\|_{L^{2}}\|\phi\|_{H^{1}} \eta,
$$

and for the smooth part $\psi_{\eta} * \phi$ we use the equation (77) to get

$$
\begin{aligned}
\left|\left(\delta f_{s t}, \psi_{\eta} * \phi\right)\right| & \lesssim(t-s)\left\|f_{0}\right\|_{L^{2}}\|a\|_{W^{1, \infty}}\left\|\psi_{\eta} * \phi\right\|_{H^{2}}+\|\mathbf{B}\|_{\alpha}\left\|f_{0}\right\|_{L^{2}}\left\|\psi_{\eta} * \phi\right\|_{H^{1}}+\|\mathbf{B}\|_{\alpha}\left\|f_{0}\right\|_{L^{2}}\left\|\psi_{\eta} * \phi\right\|_{H^{2}} \\
& +|t-s|^{3 \alpha} C\left\|f_{0}\right\|_{L^{2}}\left\|\psi_{\eta} * \phi\right\|_{H^{3}} \\
& \leq\left\|f_{0}\right\|_{L^{2}} C\left[(t-s) \eta^{-1}+|t-s|^{\alpha}+|t-s|^{2 \alpha} \eta^{-1}+|t-s|^{3 \alpha} \eta^{-2}\right]\|\phi\|_{H^{1}} .
\end{aligned}
$$

Choosing $\eta=|t-s|^{\alpha}$ we get the second inequality in (75). 


\subsection{Existence of a smooth solution}

With the previous a priori estimates at hand, we are ready to prove existence of a solution.

Theorem 5.8. Let Assumption 5.1 hold for $n>6+\frac{d}{2}$ and let $u_{0} \in C_{c}^{\infty}\left(\mathbb{R}^{d}\right)$ be given. Then there exists a solution to (63) which belongs to $C_{b}^{6}$ and

$$
\delta u_{s t}=\int_{s}^{t} \operatorname{div}\left(a_{r} \nabla u_{r}\right) d r+B_{s t}^{1} u_{s}+B_{s t}^{2} u_{s}+u_{s t}^{\natural}
$$

holds in $C_{b}^{3}$ in the sense that $u^{\natural} \in C_{2}^{3 \alpha}\left([0, T] ; C_{b}^{3}\left(\mathbb{R}^{d}\right)\right)$, where $\mathbf{B}=\left(B^{1}, B^{2}\right)$ is the unbounded rough driver constructed from $\mathbf{X}$ as in Proposition 5.3

Proof. Denote by $u^{\epsilon}$ the solution of (63) when $X$ is replaced by $X^{\epsilon}$, which we write

$$
\delta u_{s t}^{\epsilon}=\int_{s}^{t} \operatorname{div}\left(a \nabla u_{r}^{\epsilon}\right) d r+X_{s t}^{\epsilon} \nabla u_{s}^{\epsilon}+\int_{s}^{t} \dot{X}_{r}^{\epsilon} \nabla\left(X_{s r}^{\epsilon} \nabla u_{s}^{\epsilon}\right) d r+u_{s t}^{\epsilon, \natural}
$$

Setting $f^{\epsilon}=\left(u^{\epsilon}, \ldots, \nabla^{n} u^{\epsilon}\right)$ and choosing $N$ large (in fact $N=1+d+\cdots+d^{n}$ ) we see that (71) is on the form (73) where $V^{\epsilon}$ and $Y^{\epsilon}$ are defined from $X^{\epsilon}$ using (72). We then build the unbounded rough driver $\mathbf{B}^{\epsilon, \otimes 2}$ and $\mathbf{B}^{\epsilon}$ from $V^{\epsilon}$ and $Y^{\epsilon}$ according to (76) and (78) respectively.

By the assumptions on $a, \mathbf{X}$ and $u_{0}$ we get

$$
\sup _{t \in[0, T]}\left\|u_{t}^{\epsilon}\right\|_{H^{n}}^{2}=\sup _{t \in[0, T]} \sum_{k=0}^{n}\left\|\nabla^{k} u_{t}^{\epsilon}\right\|_{L^{2}\left(\mathbb{R}^{d}\right)}^{2}=\sup _{t \in[0, T]}\left\|f_{t}^{\epsilon}\right\|_{L^{2}\left(\mathbb{R}^{d} ; T^{(n)}\left(\mathbb{R}^{d}\right)\right)}^{2} \leq C \sup _{t \in[0, T]}\left\|f_{0}\right\|_{L^{2}\left(\mathbb{R}^{d} ; T^{(n)}\left(\mathbb{R}^{d}\right)\right)}^{2}=C\left\|u_{0}\right\|_{H^{n}}^{2}
$$

for some constant $C$. For $\phi \in H^{n+1}$, define $\Phi \in L^{2}\left(\mathbb{R}^{d} ; T^{(n)}\left(\mathbb{R}^{d}\right)\right)$ by $\Phi=\left(\phi, \nabla \phi, \ldots, \nabla^{n} \phi\right)$ and notice

$$
\begin{aligned}
\left(\delta u_{s t}^{\epsilon}, \phi\right)_{H^{n}} & =\sum_{k=0}^{n}\left(\delta \nabla^{k} u_{s t}^{\epsilon}, \nabla^{k} \phi\right)_{L^{2}\left(\mathbb{R}^{d}\right)}=\left(\delta f_{s t}^{\epsilon}, \Phi\right)_{L^{2}\left(\mathbb{R}^{d} ; T^{(n)}\left(\mathbb{R}^{d}\right)\right)} \\
& \leq C|t-s|^{\alpha}\|\Phi\|_{H^{1}\left(\mathbb{R}^{d} ; T^{(n)}\left(\mathbb{R}^{d}\right)\right)} \leq C|t-s|^{\alpha}\|\phi\|_{H^{n+1}\left(\mathbb{R}^{d}\right)}
\end{aligned}
$$

Since $H^{n+1}$ and $H^{n-1}$ are dual w.r.t. to the inner product on $H^{n}$, we get $\left\|\delta u^{\epsilon}\right\|_{H^{n-1}\left(\mathbb{R}^{d}\right)} \leq C|t-s|^{\alpha}$. By similar reasoning we get $\left\|u_{s t}^{\natural}\right\|_{H^{n-3}\left(\mathbb{R}^{d}\right)} \leq C|t-s|^{3 \alpha}$ using (79).

Since $u^{\epsilon}$ lies in a bounded set of $C^{\alpha}\left([0, T] ; H^{n-1}\left(\mathbb{R}^{d}\right)\right) \cap C\left([0, T] ; H^{n}\left(\mathbb{R}^{d}\right)\right)$, by Arzelà-Ascoli there exists a subsequence $u^{k}:=u^{\epsilon_{k}}$ converging in $C\left([0, T] ; H_{w}^{n}\left(\mathbb{R}^{d}\right)\right)$ some element $u$. Here $H_{w}^{n}\left(\mathbb{R}^{d}\right)$ denotes $H^{n}\left(\mathbb{R}^{d}\right)$ equipped with the weak topology. Choosing now $n>6+\frac{d}{2}$ and using Sobolev embedding [4, Corollary 9.13] we get that $u^{\epsilon, \natural}$ is bounded in $C_{2}^{3 \alpha}\left([0, T] ; C_{b}^{3}\left(\mathbb{R}^{d}\right)\right)$ and $u \in C\left([0, T] ; C_{b}^{6}\left(\mathbb{R}^{d}\right)\right)$.

It is straightforward to take the limit in (81) and use the uniform bounds on $u^{\epsilon, \natural}$ to obtain (80).

\subsection{Uniqueness}

Theorem 5.9. Let Assumption 5.1 hold for $n>6+\frac{d}{2}$. Then solutions of (60) are unique.

Proof. Let $v$ be a solution to $(60)$, i.e. for all $\phi \in C_{b}^{3}$ we have

$$
\delta v_{s t}(\phi)=\int_{s}^{t} v_{r}\left(\operatorname{Tr}\left(a_{r} \nabla^{2} \phi\right)\right) d r-v_{s}\left(B_{s t}^{1} \phi\right)+v_{s}\left(B_{s t}^{2} \phi\right)+v_{s t}^{\natural}(\phi)
$$


where $v^{\natural} \in C_{2}^{3 \alpha}\left([0, T] ;\left(C_{b}^{3}\left(\mathbb{R}^{d}\right)\right)^{*}\right)$ and $\mathbf{B}=\left(B^{1}, B^{2}\right)$ is the unbounded rough driver constructed from $\mathbf{X}$. Let $u$ be the solution of the backward equation (61) with final condition $\psi \in C_{c}^{\infty}\left(\mathbb{R}^{d}\right)$ so that

$$
\delta u_{s t}=-\int_{s}^{t} \operatorname{Tr}\left(a_{r} \nabla^{2} u_{r}\right) d r+B_{s t}^{1} u_{s}+B_{s t}^{2} u_{s}+u_{s t}^{\natural}
$$

holds in $C_{b}^{3}$. We then have

$$
\begin{aligned}
\delta v(u)_{s t} & \left.=\delta v_{s t}\left(u_{s}\right)+v_{s}\left(\delta u_{s t}\right)+\delta v_{s t}\left(\delta u_{s t}\right)=\int_{s}^{t} v_{r}\left(\operatorname{Tr}\left[a_{r} \nabla^{2} u_{s}\right]\right) d r+v_{s}\left(-B_{s t}^{1} u_{s}+B_{s t}^{2} u_{s}\right]\right)+v_{s t}^{\natural}\left(u_{s}\right) \\
& -\int_{s}^{t} v_{s}\left(\operatorname{Tr}\left[a_{r} \nabla^{2} u_{r}\right]\right) d r+v_{s}\left(B_{s t}^{1} u_{s}\right)+v_{s}\left(B_{s t}^{2} u_{s}\right)+v_{s}\left(u_{s t}^{\natural}\right) \\
& +v_{s t}^{\sharp}\left(u_{s t}^{\sharp}\right)+v_{s t}^{\sharp}\left(B_{s t}^{1} u_{s}\right)-v_{s}\left(B_{s t}^{1} u_{s t}^{\sharp}\right)-v_{s}\left(B_{s t}^{1} B_{s t}^{1} u_{s}\right) \\
& =\int_{s}^{t} \delta v_{s r}\left(\operatorname{Tr}\left[a_{r} \nabla^{2} u_{r}\right]\right) d r-\int_{s}^{t} v_{r}\left(\operatorname{Tr}\left[a_{r} \delta \nabla^{2} u_{s r}\right]\right) d r \\
& +v_{s t}^{\natural}\left(u_{s}\right)+v_{s}\left(u_{s t}^{\natural}\right)+v_{s t}^{\sharp}\left(u_{s t}^{\sharp}\right)+v_{s t}^{\sharp}\left(B_{s t}^{1} u_{s}\right)-v_{s}\left(B_{s t}^{1} u_{s t}^{\sharp}\right)
\end{aligned}
$$

where we have defined

$$
v_{s t}^{\sharp}:=\delta v_{s t}+B_{s t}^{1, *} v_{s}, \quad u_{s t}^{\#}:=\delta u_{s t}-B_{s t}^{1} u_{s}
$$

and we have used that the path is geometric which gives $v_{s}\left(B_{s t}^{1} B_{s t}^{1} u_{s}\right)=v_{s}\left(B_{s t}^{2} u_{s}\right)$. Using the equations for $u$ and $v$ we get $v^{\sharp} \in C_{2}^{2 \alpha}\left([0, T] ;\left(C_{b}^{3}\left(\mathbb{R}^{d}\right)\right)^{*}\right)$ and $u^{\sharp} \in C_{2}^{2 \alpha}\left([0, T] ; C_{b}^{3}\left(\mathbb{R}^{d}\right)\right)$. Using this and analyzing every term in $(82)$ we see that

$$
\left|\delta v(u)_{s t}\right| \lesssim|t-s|^{3 \alpha}, \quad \Longrightarrow \quad v_{t}\left(u_{t}\right)=\text { const. }
$$

and in particular $v_{T}(\psi)=v_{0}\left(u_{0}\right)$. If $\bar{v}$ is any other solution with the same initial condition, the same analysis gives $\bar{v}_{T}(\psi)=v_{0}\left(u_{0}\right)$ which gives that $\nu_{T}(\psi)=\bar{v}_{T}(\psi)$. Since $\psi$ was arbitrary the result follows.

\section{The McKean-Vlasov equation}

Let $d, m \in \mathbb{N}$ be fixed. Let $\left(\Omega, \mathcal{F},\left(\mathcal{F}_{t}\right)_{t \in[0, T]}, P\right)$ be a complete filtered probability space and $W$ be a $d$-dimensional Wiener process on it. Let $\Xi: \Omega \rightarrow \mathbb{R}^{d}$ be an $\mathcal{F}_{0}$-measurable random variable. Let $\mathbf{Z} \in \mathscr{C}_{g}^{\bar{\alpha}}\left([0, T], \mathbb{R}^{m}\right)$, for $\bar{\alpha} \in\left(\frac{1}{3}, \frac{1}{2}\right)$. Moreover let $\alpha \in\left(\frac{1}{3}, \bar{\alpha}\right)$ and $p=\frac{1}{\alpha}$.

In this section we prove well-posedness of the equation

$$
d x_{t}=\sigma\left(\mathcal{L}\left(x_{t}\right), x_{t}\right) d W_{t}+\beta\left(\mathcal{L}\left(x_{t}\right), x_{t}\right) d \mathbf{Z}_{t}, \quad x_{0}=\Xi \in \mathbb{R}^{d} .
$$

We start by defining the notion of solution we shall use.

Definition 6.1. Let $\rho \geq 1$ and $\alpha \in\left(\frac{1}{3}, \frac{1}{2}\right]$. We say that an $\left(\mathcal{F}_{t}\right)_{t \geq 0}$-adapted stochastic process $x$ : $\Omega \times[0, T] \rightarrow \mathbb{R}^{d}$ is a solution to equation (83) with initial condition $\Xi \in L^{\rho}\left(\Omega, \mathcal{F}_{0} ; \mathbb{R}^{d}\right)$, if

(i) $\mu_{t}:=\mathcal{L}\left(x_{t}\right)$ is such that

$$
(\mu(\beta), \mu(\nabla \beta \mu(\beta))) \in \mathscr{D}_{Z}^{2 \alpha}\left([0, T] ; H^{k}\right) .
$$

and $\mathbf{F}^{\mu}$ defined from $\sigma(\mu)$ and $\beta(\mu)$ as in Lemma 4.8 is a rough driver in the sense of Definition (3.1). 
(ii) P-almost surely, $x$ satisfies

$$
d x_{t}=\mathbf{F}_{d t}^{\mu}\left(x_{t}\right), \quad x_{0}=\Xi
$$

in the sense of Definition 3.4

Before proceeding we state the assumptions that will be in force throughout the section.

Assumptions 6.2. Let $k>\frac{d}{2}+3$ and $\rho \geq 1$,

(i) We assume $\beta \in \mathcal{L}\left(\mathbb{R}^{m}, C_{b}^{3} \otimes H^{k}\right)$.

(ii) Let $\sigma: \mathcal{P}_{\rho}\left(\mathbb{R}^{d}\right) \rightarrow \mathcal{L}\left(\mathbb{R}^{d} ; H^{k}\right)$ be a measurable function, such that there exists a constant $C_{\sigma}>0$, with

$$
\|\sigma(\mu)-\sigma(v)\|_{\mathcal{L}\left(\mathbb{R}^{d} ; H^{k}\right)} \leq C_{\sigma} W_{\rho}(\mu, v), \quad\|\sigma(\mu)\|_{\mathcal{L}\left(\mathbb{R}^{d} ; H^{k}\right)} \leq C_{\sigma}, \quad \forall \mu, v \in \mathcal{P}_{\rho}\left(\mathbb{R}^{d}\right) .
$$

We now introduce a suitable space of measures in which will be useful for proving well-posedness of (83). The set up is reminiscent of the controlled space as introduced in [17], but tailored for measures on path spaces.

Definition 6.3. Let $\rho \geq 1$. We say that a pair $(\mu, \gamma) \in \mathcal{P}_{\rho}\left(C_{0}^{\alpha}\left([0, T] ; \mathbb{R}^{d}\right)\right) \times C^{\alpha}\left([0, T] ; \mathcal{L}\left(\mathbb{R}^{m} ; C_{b}^{3}\left(\mathbb{R}^{d} ; \mathbb{R}^{d}\right)\right)\right.$ is controlled by $Z$ provided for every $\phi \in C_{b}^{3} \otimes H^{k}$ we have that

$$
\left(\mu(\phi), \mu\left(\nabla_{1} \phi \gamma\right)\right) \in \mathscr{D}_{Z}^{2 \alpha}\left([0, T] ; H^{k}\right) .
$$

Here we used the notation

$$
\mu(\phi)_{t}=\int_{C^{\alpha}} \phi\left(\omega_{t}, \cdot\right) d \mu(\omega), \quad \mu\left(\nabla_{1} \phi \gamma\right)_{t}^{j}=\int_{C^{\alpha}} \nabla_{1} \phi\left(\omega_{t}, \cdot\right) \gamma_{t}^{j}\left(\omega_{t}\right) d \mu(\omega) .
$$

For $\rho \geq 1$, we denote by $\mathcal{M}_{Z}^{2 \alpha, \rho}$ the set of all such controlled pairs equipped with the metric

$$
d((\mu, \gamma),(v, \zeta))=W_{\rho}(\mu, v)+[\gamma-\zeta]_{\alpha ; C_{b}^{3}}+\sup _{\|\phi\|_{C_{b}^{3} \otimes H^{k}} \leq 1}\left\|\left(\mu(\phi)-v(\phi), \mu\left(\nabla_{1} \phi \gamma\right)-v\left(\nabla_{1} \phi \zeta\right)\right)\right\|_{Z, \alpha ; H^{k}} .
$$

Remark 6.4. We note that in Definition 6.1](i) the law, $\mu_{t}=\mathcal{L}\left(x_{t}\right)$, of the solution is only defined for the time-marginals, and a priori it is not clear how to construct from this a measure on the path space $C_{0}^{\alpha}\left([0, T] ; \mathbb{R}^{d}\right)$. However, since $x$ satisfies the equation in Definition 6.1](ii) $x$ is a random variable in $C^{\alpha}\left([0, T] ; \mathbb{R}^{d}\right)$, and letting $h \rightarrow 0$ in (25) and (49) we see that $x$ takes values in $C_{0}^{\alpha}\left([0, T] ; \mathbb{R}^{d}\right)$. Hence it induces the measure $\mathcal{L}(x)$ on $C_{0}^{\alpha}\left([0, T] ; \mathbb{R}^{d}\right)$ which clearly has time-marginals $\mu_{t}$.

Remark 6.5. Let $\beta$ and $\sigma$ satisfy Assumption 6.2 with $k>\frac{d}{2}+3$, and let $(\mu, \gamma) \in \mathcal{M}_{Z}^{2 \alpha, \rho}$. Then, $\sigma(\mu)$ and $\mu(\beta), \mu\left(\nabla_{1} \beta \gamma\right)$ satisfy Assumption 4.2 Assumption 4.2[(i) is verified by replacing $\varphi=\beta^{i}$, for $i=1, \ldots, m$, in Definition 6.3 Assumption 4.2](ii) follows trivially by the boundedness in Assumption 4.2[(ii)] We are only left with verifying 4.2[(iii)] For all $s, t \in[0, T]$,

$\left\|\sigma\left(\mu_{t}\right)-\sigma\left(\mu_{s}\right)\right\|_{\mathcal{L}\left(\mathbb{R}^{d} ; H^{k}\right)} \leq C_{\sigma} W_{\rho}\left(\mu_{t}, \mu_{s}\right) \leq C_{\sigma}\left(\int_{C_{0}^{\alpha}}\left|\omega_{t}-\omega_{s}\right|^{\rho} d \mu(\omega)\right)^{\frac{1}{\rho}} \leq C_{\sigma}\left(\int_{C_{0}^{\alpha}}[\omega]_{\alpha}^{\rho} d \mu(\omega)\right)^{\frac{1}{\rho}}|t-s|^{\alpha}$.

This gives that $\sigma \in C^{\alpha} H^{k} \subset C^{p-v a r} H^{k}$, if $p=\frac{1}{\alpha}$. 
Theorem 6.6. Suppose $\sigma$ and $\beta$ satisfies Assumption 6.2 and $\rho \geq 2$. For any $\Xi_{0} \in L^{\rho}\left(\Omega, \mathcal{F}_{0} ; \mathbb{R}^{d}\right)$ there exists a unique solution $x$ of (83) in the sense of Definition 6.1

Proof. We fix $\sigma, \beta$ satisfying Assumptions 6.2 and construct the following mappings

$$
\begin{array}{ccccccc}
\mathcal{M}_{Z}^{2 \alpha, \rho} & \rightarrow & C^{\alpha}\left([0, T] ; H^{k}\right) \times \mathscr{D}_{Z}^{2 \alpha}\left([0, T] ; H^{k}\right) & \rightarrow & \mathscr{C}^{\alpha} & \rightarrow & \mathcal{M}_{Z}^{2 \alpha, \rho} \\
(\mu, \gamma) & \mapsto & \left(\sigma(\mu),\left(\beta(\mu), \beta(\mu)^{\prime}\right)\right) & \mapsto & \mathbf{F}^{\mu} & \mapsto & (\mathcal{L}(x), \beta(\mu)) .
\end{array}
$$

and we shall use the notation $\Gamma(\mu, \gamma):=(\mathcal{L}(x), \beta(\mu))$. By letting $h \rightarrow 0$ in 25) and (49) we see that $\mathcal{L}(x)$ is supported on $C_{0}^{\alpha}\left([0, T] ; \mathbb{R}^{d}\right)$. In Lemma 6.7 and Lemma 6.8 we show that $\Gamma$ is a contraction mapping on a subset of $\mathcal{M}_{Z}^{2 \alpha, \rho}$ for a small time parameter $T_{0} \leq T$. Then, noting that $T_{0}=T_{0}(\rho, \alpha, \sigma, \beta, \mathbf{Z})$ does not depend on the initial condition $\Xi_{0}$, the solution can be constructed iteratively on the full time interval $[0, T]$ by concatenation of the solutions defined on $\left[0, T_{0}\right],\left[T_{0}, 2 T_{0}\right]$ etc.

\section{Lemma 6.7. Define}

$$
\bar{L}=\bar{L}(\sigma, \beta, \mathbf{Z}):=\left(1+C_{\sigma}+\|\beta\|_{C_{b}^{3} \otimes H^{k}}\right)\left(1+[\mathbf{Z}]_{\bar{\alpha}} \vee[\mathbf{Z}]_{\bar{\alpha}}^{\frac{1}{2}}\right),
$$

and the closed subset of $\mathcal{M}_{Z}^{2 \alpha, \rho}$,

$$
\mathcal{B}_{T}:=\left\{(\mu, \gamma) \in \mathcal{M}_{Z}^{2 \alpha, \rho} \mid d\left((\mu, \gamma),\left(\mu, \delta_{0}\right)\right) \leq 1, \quad W_{\rho}\left(\mu, \delta_{0}\right) \leq \frac{1}{3} \bar{L}^{-1}\right\}
$$

Assume Assumption 6.2 with $\rho \geq 2$. There exists a small time $T=T(\rho, \alpha, \sigma, \beta, \mathbf{Z})$, such that $\Gamma$ leaves $\mathcal{B}_{T}$ invariant.

Proof. We start by looking at the controlled function,

$$
\begin{aligned}
\left\|\delta \beta(\mu)_{s t}\right\|_{H^{k}} & =\left\|\int_{C_{0}^{\alpha}} \delta \beta(\omega ., \cdot)_{s t} d \mu(\omega)\right\|_{H^{k}} \leq\|\beta\|_{C_{b}^{3} \otimes H^{k}} \int_{C_{0}^{\alpha}}\left|\delta \omega_{s t}\right| d \mu(\omega) \\
& \leq\|\beta\|_{C_{b}^{3} \otimes H^{k}} \int_{C_{0}^{\alpha}}[\omega]_{\alpha} d \mu(\omega)|t-s|^{\alpha} \leq\|\beta\|_{C_{b}^{3} \otimes H^{k}} W_{\rho}\left(\mu, \delta_{0}\right)|t-s|^{\alpha} \leq \frac{1}{3}|t-s|^{\alpha}
\end{aligned}
$$

To show the bounds on the rough driver, start by noting that, by linearity,

$$
\left\|\beta(\mu), \beta(\mu)^{\prime}\right\|_{Z, \alpha ; H^{k}} \leq\|\beta\|_{C_{b}^{3} \otimes H^{k}} \sup _{\|\phi\|_{C^{3} \otimes H^{k}} \leq 1}\left\|\mu(\phi), \mu\left(\nabla_{1} \phi \gamma\right)\right\|_{Z, \alpha ; H^{k}} \leq\|\beta\|_{C_{b}^{3} \otimes H^{k}}
$$

and thanks to (84), $\|\sigma(\mu)\|_{L_{t}^{\infty} \mathcal{L}\left(\mathbb{R}^{d} ; H^{k}\right)} \leq C_{\sigma}$. This gives that for $(\mu, \gamma) \in \mathcal{B}_{T}$, we have $L(\sigma(\mu), \beta(\mu), \mathbf{Z}) \leq$ $\bar{L}(\sigma, \beta, \mathbf{Z})$, where $L$ is defined in (36). The previous observation and (49) imply

$$
\left[\mathbf{F}^{\mu}\right]_{\alpha}=\left[\mathbf{F}^{\mu}\right]_{\alpha ; C_{b}^{3}} \leq \bar{L} T^{\frac{\bar{\alpha}-\alpha}{2}} K_{\rho}
$$

for any $\alpha<\bar{\alpha}$ and for any $\rho \geq 1$ and for a random variable $K_{\rho} \in L^{\rho}(\Omega)$. From the a priori estimates (26) we see that there exists a constant $C>0$, depending only on $\rho$ (which may change from an inequality to the next), such that

$$
\left\|[x]_{\alpha}\right\|_{L_{\omega}^{\rho}} \leq C\left(E\left[[\mathbf{F}]_{\alpha}^{\rho} \vee[\mathbf{F}]_{\alpha}^{\rho / \alpha}\right]\right)^{1 / \rho} \leq C T^{\frac{\bar{\alpha}-\alpha}{2}} \bar{L}^{1 / \alpha} .
$$


We may now choose $T \leq\left(3 C \bar{L}^{1+1 / \alpha}\right)^{-\frac{2}{\bar{\alpha}-\alpha}}$ such that

$$
\bar{L}\left\|[x]_{\alpha}\right\|_{L_{\omega}^{\rho}} \leq \frac{1}{3} .
$$

From Lemma 4.16 we get,

$$
\sup _{\|\phi\|_{C_{b}^{3} \otimes H^{k}} \leq 1}\left\|\left(\mathcal{L}(x)(\phi), \mathcal{L}(x)\left(\nabla_{1} \phi \beta(\mu)\right)\right)\right\|_{Z, \alpha ; H^{k}} \leq T^{\frac{\bar{\alpha}-\alpha}{2}}\left(1+\left\|[x]_{\alpha}\right\|_{L_{\omega}^{2}}\right) \bar{L} \leq T^{\frac{\bar{\alpha}-\alpha}{2}}\left(1+C \bar{L}^{1 / \alpha}\right) \bar{L} .
$$

and we choose $T \leq\left(3(1+C)\left(1+\bar{L}^{1+1 / \alpha}\right)\right)^{-\frac{2}{\alpha-\alpha}}$ such that the above is bounded by $\frac{1}{3}$. This shows that

$$
d\left(\Gamma(\mu, \gamma),\left(\delta_{0}, 0\right)\right)=\left\|[x]_{\alpha}\right\|_{L_{\omega}^{\rho}}+[\beta(\mu)]_{\alpha ; C^{3}}+\sup _{\|\phi\|_{C_{b}^{3} \otimes H^{k}} \leq 1}\left\|\left(\mathcal{L}(x)(\phi), \mathcal{L}(x)\left(\nabla_{1} \phi \beta(\mu)\right)\right)\right\|_{Z, \alpha ; H^{k}} \leq \frac{1}{3} .
$$

This, together with (87) implies $\Gamma\left(\mathcal{B}_{T}\right) \subset \mathcal{B}_{T}$.

Lemma 6.8. Assume Assumption 6.2 with $\rho \geq 2$. There exists a constant $0<c<1$ and a small time $T=T(\rho, \alpha, \sigma, \beta, \mathbf{Z})$, such that, for all $(\mu, \gamma),(\nu, \zeta) \in \mathcal{B}_{T}$, we have

$$
d(\Gamma(\mu, \gamma), \Gamma(v, \zeta)) \leq c d((\mu, \gamma),(v, \zeta))
$$

Proof. Let $M=K\left(\llbracket \sigma \rrbracket_{p,[s, t]}^{\rho}+1\right)(L(\sigma, \beta, \mathbf{Z})+L(\theta, \gamma, \mathbf{Z}))$ be defined as in Lemma 4.17, We have seen in the proof of Lemma 6.7 that, for $(\mu, \gamma) \in \mathcal{B}_{T}$, we have $L(\sigma(\mu), \beta(\mu), \mathbf{Z}) \leq \bar{L}(\sigma, \beta, \mathbf{Z})$. Moreover, from (84), we have $\llbracket \sigma(\mu) \rrbracket_{p}^{p} \leq C_{\sigma} T\left(\int_{C_{0}^{\alpha}}[\omega]_{\alpha}^{\rho} d \mu(\omega)\right)^{\frac{1}{\rho}} \leq \frac{T}{3}$, for $\mu \in \mathcal{B}_{T}$. Hence $M \leq K \bar{L}$, for some universal constant $K=K(\alpha, \rho)$. We estimate the Wasserstein distance of the image laws, as given in (85). From Lemma4.17, there exists $\bar{\rho} \geq \rho$ and $C(T)>0$, such that $\lim _{T \rightarrow 0} C(T)=0$ and

$$
\begin{aligned}
W_{\rho}(\mathcal{L}(x), \mathcal{L}(y)) \leq\left\|[x-y]_{\alpha}\right\|_{L_{\omega}^{\rho} \leq} & C(T) e^{M^{\bar{\rho}}}\left(\|\sigma(\mu)-\sigma(v)\|_{L_{t}^{\infty} \mathcal{L}\left(\mathbb{R}^{d} ; H^{k}\right)}\right. \\
& \left.+\left\|\left(\beta(\mu), \beta(\mu)^{\prime}\right)-\left(\beta(v), \beta(v)^{\prime}\right)\right\|_{Z, \alpha ; H^{k}}\right) \\
\leq & C(T) e^{(K \bar{L})^{\bar{p}}} d((\mu, \gamma),(\nu, \zeta)) .
\end{aligned}
$$

We study now the Gubinelli derivative. For all $s, t \in[0, T]$, we have

$$
\begin{aligned}
\left\|\beta(\mu)_{s t}-\beta(v)_{s t}\right\|_{H^{k}} & \leq\left\|\left(\mu(\nabla \beta \gamma)_{s}-v(\nabla \beta \zeta)_{s}\right) Z_{s t}\right\|_{H^{k}}+\left\|\mu(\beta)_{s t}^{\#}-\zeta(\beta)_{s t}^{\#}\right\|_{H^{K}} \\
& \leq\|(\mu(\beta), \mu(\nabla \beta \gamma))-(v(\beta), v(\nabla \beta \zeta))\|_{Z, \alpha, H^{k}}\left([\mathbf{Z}]_{\alpha}+|t-s|^{2 \alpha}\right) .
\end{aligned}
$$

Hence, using $\bar{\alpha}>\alpha$ and $\bar{L} \geq L$,

$$
[\beta(\mu)-\beta(v)]_{\alpha ; C_{b}^{3}} \leq[\beta(\mu)-\beta(v)]_{\alpha ; H^{k}} \leq\|\beta\|_{C_{b}^{3} \otimes H^{k}} T^{\bar{\alpha}-\alpha} \bar{L} d((\mu, \gamma),(\nu, \zeta)) .
$$

For the last term in the definition of the metric $d$, we have, using Proposition 4.17 and proceeding as in (89)

$\sup _{\|\phi\|_{C_{b}^{3} \otimes H^{k}} \leq 1}\left\|\left(E[\phi(x)], E\left[\nabla_{1} \phi(x) \beta(x)\right]\right)-\left(E[\phi(y)], E\left[\nabla_{1} \phi(y) \gamma(y)\right]\right)\right\|_{Z, \alpha, H^{k}} \leq C(T) e^{(K \bar{L})^{\bar{p}}} d((\mu, \gamma),(v, \zeta))$.

We now add together (89), 90), and (91) to obtain

$$
d(\Gamma(\mu, \gamma), \Gamma(\nu, \zeta)) \leq C(T) e^{(K \bar{L})^{\bar{\rho}}} d((\mu, \gamma),(\nu, \zeta)) .
$$

Choosing $T=T(\rho, \alpha, \sigma, \beta, \mathbf{Z})$ small enough, depending on $\bar{L}$, we conclude the proof. 


\section{Non local rough PDEs}

Let $d, m \in \mathbb{N}$ be fixed. Let $\mathbf{Z} \in \mathscr{C}_{g}^{\bar{\alpha}}\left([0, T], \mathbb{R}^{m}\right)$, for $\bar{\alpha} \in\left(\frac{1}{3}, \frac{1}{2}\right)$. Moreover let $\alpha \in\left(\frac{1}{3}, \bar{\alpha}\right)$ and $p=\frac{1}{\alpha}$. Let $\sigma$ and $\beta$ satisfy Assumption 6.2 .

We turn to the Fokker-Planck equation induced by the rough diffusion, which formally reads

$$
\partial_{t} \mu=\frac{1}{2} \operatorname{Tr} \nabla^{2}\left(\left[\sigma(\mu) \sigma(\mu)^{T} \mu\right]\right)-\operatorname{div}(\beta(\mu) \mu) \dot{Z}, \quad \mu_{0} \in \mathcal{P}\left(\mathbb{R}^{d}\right) .
$$

We define the notion of a solution in a similar way as in the linear case, Definition 5.4, but where now the unbounded rough driver depends on the solution itself.

Definition 7.1. We say that a path $\mu:[0, T] \rightarrow \mathcal{P}_{\rho}\left(\mathbb{R}^{d}\right)$ is a solution of with initial condition $\mu_{0} \in \mathcal{P}_{\rho}\left(\mathbb{R}^{d}\right)$ provided

(i) for all $\varphi \in C_{b}^{3} \otimes H^{k}$,

$$
\left(\mu(\varphi), \mu(\nabla \varphi \beta(\mu)) \in \mathscr{D}_{Z}^{2 \alpha}\left([0, T] ; H^{k}\right) .\right.
$$

(ii) $\mu$ satisfies (69) with the unbounded rough driver $\mathbf{B}=\mathbf{B}^{\mu}$ defined from

$$
X_{s t}^{\mu}=\int_{s}^{t} \beta\left(\mu_{r}, \cdot\right) d \mathbf{Z}_{r}, \quad \mathbb{X}_{s t}^{\mu}=\int_{s}^{t} \beta\left(\mu_{r}, \cdot\right) \int_{s}^{r} \beta\left(\mu_{u}, \cdot\right) d \mathbf{Z}_{u} d \mathbf{Z}_{r}
$$

as in Proposition 5.3 and $a_{t}=\frac{1}{2} \sigma\left(\mu_{t}\right) \sigma\left(\mu_{t}\right)^{T}$.

Existence of a solution to (92) is relatively straightforward.

Theorem 7.2. Suppose $\sigma$ and $\beta$ satisfies Assumptions $6.2 \mu_{0} \in \mathcal{P}_{\rho}\left(\mathbb{R}^{d}\right)$ for $\rho \geq 2$ and $\mathbf{Z} \in C_{w g}^{\bar{\alpha}}\left([0, T] ; \mathbb{R}^{m}\right)$ for $\bar{\alpha} \in\left(\frac{1}{3}, \frac{1}{2}\right)$. Let $\left(\Omega, \mathcal{F},\left(\mathcal{F}_{t}\right)_{t \in[0, T]}, P\right)$ be a complete probability space that supports a d-dimensional Brownian motion $W$ and an $\mathcal{F}_{0}$-measurable random variable, $\Xi \in L^{\rho}\left(\Omega ; \mathbb{R}^{d}\right)$ such that the pushforward measure $P_{*}(\Xi)=\mu_{0}$. Then, there exists a solution $\mu$ of (92), in the sense of Definition 7.1$]$ This solution is given by $\mu_{t}=\mathcal{L}\left(x_{t}\right)$, where $x$ is the unique solution to the McKean-Vlasov equation (83) with initial condition $\Xi$, in the sense of Definition 6.1

Proof. The proof is completed by following the same steps as in Proposition 5.5 except the unbounded rough driver depends on the solution itself.

The following result will be crucial for proving uniqueness of the non-local Fokker-Planck equation.

Proposition 7.3. Let $\bar{\alpha} \in\left(\frac{1}{3}, \frac{1}{2}\right), \alpha \in\left(\frac{1}{3}, \bar{\alpha}\right)$ and $\mathbf{Z} \in \mathscr{C}_{w g}^{\bar{\alpha}}\left([0, T], \mathbb{R}^{m}\right)$ is weakly geometric. Define for $(\mu, \gamma) \in \mathcal{M}_{Z}^{2 \alpha, \rho}$ and $\phi \in C_{b}^{3} \otimes H^{k}$,

$$
X_{s t}^{\phi}=\int_{s}^{t} \phi\left(\mu_{r}, \cdot\right) d \mathbf{Z}_{r}, \quad \mathbb{X}_{s t}^{\phi}=\int_{s}^{t} \phi\left(\mu_{r}, \cdot\right) \int_{s}^{r} \phi\left(\mu_{u}, \cdot\right) d \mathbf{Z}_{u} d \mathbf{Z}_{r} .
$$

Then $\mathbf{X}^{\phi} \in \mathscr{C}_{g}^{\alpha}\left([0, T] ; H^{k}\right)$. 
Proof. We prove this result in two steps. First we show that the controlled path $\left(\mu(\phi), \mu\left(\nabla_{1} \phi \gamma\right)\right)$ can be continuously approximated by controlled paths which takes values in a finite-dimensional space. This clearly gives that $\mathbf{X}^{\phi}$ can be approximated by a sequence of finite dimensional rough paths. In the second step we use that the finite dimensional rough path is weakly geometric to find a smooth approximation of $\mathbf{X}^{\phi}$.

Step 1. For simplicity we only show this for $\phi \in C_{b}^{3}\left(\mathbb{R}^{d}\right) \otimes L^{2}\left(\mathbb{R}^{d}\right)$, the general case follows by replacing $\phi$ by $D_{2}^{\beta} \phi$ for $|\beta| \leq k$. Let $\left\{e_{n}\right\}$ be an orthonormal basis of $L^{2}\left(\mathbb{R}^{d}\right)$ and define

$$
\phi^{N}(x, y):=\sum_{n=1}^{N}\left\langle\phi(x, \cdot), e_{n}\right\rangle e_{n}(y) .
$$

We now show that $\left(\phi^{N}(\mu), \nabla \phi^{N}(\mu) \gamma\right) \rightarrow(\phi(\mu), \nabla \phi(\mu) \gamma)$ in $\mathscr{D}_{Z}^{2 \alpha^{\prime}}\left([0, T] ; L^{2}\right)$ for any $\alpha^{\prime} \in(\alpha, \bar{\alpha})$.

Start with the first component.

$$
\begin{aligned}
\left\|\delta \phi^{N}(\mu)_{s t}-\delta \phi(\mu)_{s t}\right\|_{L^{2}}^{2} & =\sum_{n>N}\left|\left\langle\delta \phi(\mu)_{s t}, e_{n}\right\rangle\right|^{2}=\sum_{n>N}\left|\int_{\mathbb{R}^{d}} \int_{C_{0}^{\alpha}} \delta \phi(\omega, y)_{s t} e_{n}(y) d \mu(\omega) d y\right|^{2} \\
& =\sum_{n>N}\left|\int_{\mathbb{R}^{d}} \int_{C_{0}^{\alpha}} \int_{0}^{1} \nabla_{1} \phi\left(\omega_{s}+\theta \delta \omega_{s t}, y\right) \omega_{s t} e_{n}(y) d \theta d \mu(\omega) d y\right|^{2} \\
& =\sum_{n>N}\left|\int_{C_{0}^{\alpha}} \int_{0}^{1}\left\langle\nabla_{1} \phi\left(\omega_{s}+\theta \delta \omega_{s t}\right), e_{n}\right\rangle \omega_{s t} d \theta d \mu(\omega)\right|^{2} \\
& \leq \int_{C_{0}^{\alpha}} \int_{0}^{1} \sum_{n>N}\left|\left\langle\nabla_{1} \phi\left(\omega_{s}+\theta \delta \omega_{s t}\right), e_{n}\right\rangle\right|^{2}[\omega]_{\alpha}^{2} d \theta d \mu(\omega)|t-s|^{2 \alpha}
\end{aligned}
$$

Now for fixed $\omega, \theta$ and every $s, t \in[0, T]$ we have the monotone convergence

$$
\sum_{n>N}\left|\left\langle\nabla_{1} \phi\left(\omega_{s}+\theta \delta \omega_{s t}\right), e_{n}\right\rangle\right|^{2} \rightarrow 0
$$

as $N \rightarrow \infty$ since $\phi \in C_{b}^{3}\left(\mathbb{R}^{d}\right) \otimes L^{2}\left(\mathbb{R}^{d}\right)$. Moreover, for fixed $N$, as a function of $s$ and $t$ the above is continuous. By Dini's theorem we get

$$
\sup _{s, t} \sum_{n>N}\left|\left\langle\nabla_{1} \phi\left(\omega_{s}+\theta \delta \omega_{s t}\right), e_{n}\right\rangle\right|^{2} \rightarrow 0
$$

as $N \rightarrow \infty$. This gives

$$
\left[\delta \phi^{N}(\mu)-\phi(\mu)\right]_{\alpha, L^{2}}^{2} \leq \sup _{s, t} \int_{C_{0}^{\alpha}} \int_{0}^{1} \sum_{n>N}\left|\left\langle\nabla_{1} \phi\left(\omega_{s}+\theta \delta \omega_{s t}\right), e_{n}\right\rangle\right|^{2}[\omega]_{\alpha}^{2} d \theta d \mu(\omega) \rightarrow 0
$$

by monotone convergence. In a similar way one can show that $\nabla_{1} \phi^{N}(\mu \gamma)$ converges to $\nabla_{1} \phi(\mu \gamma)$ in $C^{\alpha}\left([0, T] ; L^{2}\left(\mathbb{R}^{d}\right)\right)$.

To see the convergence of the remainder, $\phi^{N}(\mu)_{s t}^{\sharp}:=\delta \phi^{N}(\mu)_{s t}-\nabla_{1} \phi^{N}\left(\mu_{s} \gamma_{s}\right) Z_{s t}$, we note first that this term is obviously bounded in $C_{2}^{2 \alpha}\left([0, T] ; L^{2}\left(\mathbb{R}^{d}\right)\right)$. Furthermore, writing

$$
\begin{aligned}
\left\|\phi^{N}(\mu)_{s t}^{\sharp}-\phi(\mu)_{s t}^{\#}\right\|_{L^{2}}^{2} & =\sum_{n>N}\left|\int_{C_{0}^{\alpha}} \delta\left\langle\phi(\omega), e_{n}\right\rangle_{s t}-\left\langle\nabla_{1} \phi\left(\omega_{s}\right), e_{n}\right\rangle \gamma_{s}\left(\omega_{s}\right) Z_{s t} d \mu(\omega)\right|^{2} \\
& \leq \int_{C_{0}^{\alpha}} \sum_{n>N}\left|\delta\left\langle\phi(\omega), e_{n}\right\rangle_{s t}-\left\langle\nabla_{1} \phi\left(\omega_{s}\right), e_{n}\right\rangle \gamma_{s}\left(\omega_{s}\right) Z_{s t}\right|^{2} d \mu(\omega) .
\end{aligned}
$$


Using Dini's theorem and monotone convergence as before we get that for any $\epsilon>0$ there exists $N_{\epsilon}$ such that for all $N \geq N_{\epsilon}$ we have $\sup _{s, t}\left\|\phi^{N}(\mu)_{s t}^{\sharp}-\phi(\mu)_{s t}^{\sharp}\right\|_{L^{2}}<\epsilon$.

This gives, uniformly in $s, t$

$$
\left\|\phi^{N}(\mu)_{s t}^{\sharp}-\phi(\mu)_{s t}^{\sharp}\right\|_{L^{2}} \leq \epsilon \wedge C|t-s|^{2 \alpha} \leq \epsilon^{1-\kappa} C^{\kappa}|t-s|^{\kappa 2 \alpha}
$$

where we have used the geometric interpolation $a \wedge b \leq a^{1-\kappa} b^{\kappa}$ for any $\kappa \in(0,1)$. By choosing $\kappa$ correctly we get $\phi^{N}(\mu)^{\sharp} \rightarrow \phi(\mu)^{\#}$ in $C_{2}^{2 \alpha^{\prime}}\left([0, T] ; L^{2}\left(\mathbb{R}^{d}\right)\right)$.

Step 2. We now proceed to prove that $\mathbf{X}^{\phi}$ can be approximated by a smooth path. Let $\epsilon>0$. From the above continuity we can choose $N$ such that

$$
\left[\mathbf{X}^{\phi^{N}}-\mathbf{X}^{\phi}\right]_{\alpha^{\prime}}<\frac{\epsilon}{2}
$$

where $\mathbf{X}^{\phi^{N}}$ is constructed by replacing $\phi$ with $\phi^{N}$ in (93).

As spelled out in Lemma A.3, there exists $\alpha<\alpha^{\prime}$ and a smooth path $X^{N, \epsilon}$ such that $\left[\mathbf{X}^{\phi^{N}}-\mathbf{X}^{N, \epsilon}\right]_{\alpha}<$ $\frac{\epsilon}{2}$. This gives

$$
\left[\mathbf{X}^{N, \epsilon}-\mathbf{X}^{\phi}\right]_{\alpha} \leq\left[\mathbf{X}^{N, \epsilon}-\mathbf{X}^{\phi^{N}}\right]_{\alpha}+\left[\mathbf{X}^{\phi^{N}}-\mathbf{X}^{\phi}\right]_{\alpha^{\prime}}<\epsilon
$$

Theorem 7.4. Suppose $\sigma, \beta$ satisfies Assumptions 6.2 for $k>9+d$ and $\mu_{0} \in \mathcal{P}_{\rho}\left(\mathbb{R}^{d}\right)$ is given with $\rho \geq 2$. Then there exists at most one solution $\mu$ of (92) in the sense of Definition 7.1

Proof. Let $\mu$ be a solution of (92). From the the assumptions on $\beta$ and $\sigma$ we may construct the timedependent coefficients $\left(\sigma(\mu),\left(\beta(\mu), \nabla_{1} \beta(\beta(\mu) \mu)\right)\right)$ from which we construct the rough driver $\mathbf{F}^{\mu}$ as in Lemma 4.8. Denote by $x^{\mu}$ the solution of

$$
d x_{t}^{\mu}=\sigma\left(\mu_{t}, x_{t}^{\mu}\right) d W_{t}+\beta\left(\mu_{t}, x_{t}^{\mu}\right) d \mathbf{Z}_{t},
$$

i.e. $d x_{t}^{\mu}=\mathbf{F}_{d t}^{\mu}\left(x_{t}\right)$. From Proposition 4.14 we see that $v$ satisfies

$$
\partial_{t} v=\frac{1}{2} \operatorname{Tr} \nabla^{2}\left(\left[\sigma(\mu) \sigma(\mu)^{T} v\right]\right)-\operatorname{div}(\beta(\mu) v) \dot{\mathbf{Z}} .
$$

as in Definition 7.1, where $X_{s t}(x)=\int_{s}^{t} \beta\left(\mu_{r}, x\right) d \mathbf{Z}_{r}$ and $\mathbb{X}_{s t}(x, y)=\int_{s}^{t} \beta\left(\mu_{r}, x\right) \int_{s}^{r} \beta\left(\mu_{u}, y\right) d \mathbf{Z}_{u} d \mathbf{Z}_{r}$. From the assumption on $\beta$, the Sobolev embedding [4, Corollary 9.13] $H^{k} \subset C_{b}^{n+3}\left(\mathbb{R}^{d} ; \mathbb{R}^{d}\right)$ for $k>$ $\frac{d}{2}+n+3$ and Proposition 7.3 we see that $\mathbf{X} \in \mathscr{C}_{g}^{\alpha}\left([0, T] ; C_{b}^{n+3}\left(\mathbb{R}^{d} ; \mathbb{R}^{d}\right)\right)$. Now if $n>6+\frac{d}{2}$, we get from Theorem 5.9 that there exists at most one solution of (94). In particular, we see that $\mu_{t}=v_{t}$ which gives that $x^{\mu}$ is a solution of (83). Since this equation is well-posed, this uniquely describes $\mu$.

\section{A Appendix}

\section{A.1 Kolmogorov continuity theorem}

In this section we prove a Kolmogorov continuity type theorem for rough drivers. The proof is done exactly as in [15, Theorem 3.1], so we only sketch the proof to convince the reader that the steps are the same. 
Theorem A.1. Suppose $\mathbf{F}=(F, \mathbb{F})$ is a random rough driver such that

$$
E\left[\left\|F_{s t}\right\|_{C_{b}^{3}}^{q}\right] \leq C|t-s|^{\beta q}, \quad E\left[\left\|\mathbb{F}_{s t}\right\|_{C_{b}^{2}}^{q / 2}\right] \leq C|t-s|^{\beta q}
$$

for $q$ and $\beta$ such that $q \beta>1$. Then for every $\alpha \in\left(0, \beta-\frac{1}{q}\right)$ we have

$$
E\left[\|F\|_{\alpha ; C_{b}^{3}}^{q}\right] \leq C, \quad E\left[\|\mathbb{F}\|_{\alpha ; C_{b}^{2}}^{q / 2}\right] \leq C
$$

and if $\beta-\frac{1}{q}>\frac{1}{3}$ then $\mathbf{F}$ is rough driver for $\alpha \in\left(\frac{1}{3}, \beta-\frac{1}{q}\right)$.

Proof. Take $T=1$ for simplicity and denote by $D_{n}$ the uniform partition of $[0,1]$ with mesh $2^{-n}$ and let

$$
K_{n}:=\sup _{t \in D_{n}}\left\|F_{t, t+2^{-n}}\right\|_{C_{b}^{3}}, \quad \mathbb{K}_{n}:=\sup _{t \in D_{n}}\left\|\mathbb{F}_{t, t+2^{-n}}\right\|_{C_{b}^{2}} .
$$

By assumption on $\mathbf{F}$ we get

$$
E\left[K_{n}^{q}\right] \leq E\left[\sum_{t \in D_{n}}\left\|F_{t, t+2^{-n}}\right\|_{C_{b}^{3}}^{q}\right] \lesssim 2^{-n(1-\beta q)}, \quad E\left[\mathbb{K}_{n}^{q / 2}\right] \leq E\left[\sum_{t \in D_{n}}\left\|\mathbb{F}_{t, t+2^{-n}}\right\|_{C_{b}^{3}}^{q / 2}\right] \lesssim 2^{-n(1-\beta q)} .
$$

Let $s, t \in \bigcup D_{n}$ and choose $m$ such that $\left|D_{m+1}\right|<|t-s| \leq\left|D_{m}\right|$. There exists a partition $\left\{t_{i}\right\}_{i=0}^{N}$ of $[s, t]$ such that $\left(t_{i}, t_{i+1}\right) \in D_{n}$ for some $n \geq m+1$, and for each fixed such $n$ there are at most two such intervals from $D_{n}$. We get

$$
\left\|F_{s t}\right\|_{C_{b}^{3}} \leq \sum_{i=0}^{N-1}\left\|F_{t_{i} t_{i+1}}\right\|_{C_{b}^{3}} \leq 2 \sum_{n \geq m+1} K_{n}
$$

and using $\mathbb{F}_{s t}=\sum_{i=0}^{N-1} \mathbb{F}_{t_{i} t_{i+1}}+\nabla F_{t_{i} t_{i+1}} F_{s t_{i}}$, which is easily seen from Chen's relation, we get

$$
\left\|\mathbb{F}_{s t}\right\|_{C_{b}^{2}} \leq \sum_{i=0}^{N-1}\left\|\mathbb{F}_{t_{i} t_{i+1}}\right\|_{C_{b}^{2}}+\left\|F_{t_{i} t_{i+1}}\right\|_{C_{b}^{3}}\left\|F_{s t_{i}}\right\|_{C_{b}^{3}} \leq 2 \sum_{n \geq m+1} \mathbb{K}_{n}+\left(2 \sum_{n \geq m+1} K_{n}\right)^{2} .
$$

This gives

$$
\frac{\left\|F_{s t}\right\|_{C_{b}^{3}}}{|t-s|^{\alpha}} \leq K_{\alpha}, \quad \frac{\left\|\mathbb{F}_{s t}\right\|_{C_{b}^{2}}}{|t-s|^{\alpha}} \leq \mathbb{K}_{\alpha}
$$

where

$$
K_{\alpha}:=2 \sum_{n \geq 0} \frac{K_{n}}{\left|D_{n}\right|^{\alpha}}, \quad \mathbb{K}_{\alpha}:=2 \sum_{n \geq 0} \frac{\mathbb{K}_{n}}{\left|D_{n}\right|^{\alpha}}
$$

which belongs to $L^{q}(\Omega)$ and $L^{q / 2}(\Omega)$ respectively. This proves the claim.

\section{A.2 Weakly geometric rough paths}

We prove that rough path integration w.r.t. a weakly geometric rough path yields a weakly geometric rough path.

Lemma A.2. Assume $\mathbf{Z}$ is weakly geometric and $E$ is a separable Hilbert space and $\left(Y, Y^{\prime}\right) \in$ $\mathscr{D}_{Z}^{2 \alpha}([0, T] ; E)$. Then the rough path $\mathbf{X}$ defined by

$$
X_{s t}:=\int_{s}^{t} Y_{r}^{k} d \mathbf{Z}_{r}^{k}, \quad \mathbb{X}_{s t}:=\int_{s}^{t} X_{r} \otimes Y_{r}^{k} d \mathbf{Z}_{r}^{k}-X_{s} \otimes X_{s t}
$$

is also weakly geometric. 
Proof. Let $\left\{e_{i}\right\}$ be an orthonormal basis of $E$ and use the component notation

$$
g^{i}=\left\langle g, e_{i}\right\rangle_{E}, \quad g \in E, \quad h^{i, j}=\left\langle h, e_{i} \otimes e_{j}\right\rangle_{E \otimes E}, \quad h \in E \otimes E .
$$

The components of the integrals may thus be spelled out

$$
X_{s t}^{i}=\int_{s}^{t} Y_{r}^{i, k} d \mathbf{Z}_{r}^{k}, \quad \mathbb{X}_{s t}^{i, j}=\int_{s}^{t} X_{r}^{i} Y_{r}^{j, k} d \mathbf{Z}_{r}^{k}-X_{s}^{i} X_{s t}^{j}
$$

where the above are scalar integrals defined by their local expansions

$$
\Xi_{s t}^{i}=Y_{s}^{i, k} Z_{s t}^{k}+Y_{s}^{i, k, l} \mathbb{Z}_{s t}^{l, k}, \quad \Xi_{s t}^{i, j}=X_{s}^{i} Y_{s}^{j, k} Z_{s t}^{k}+\left(Y_{s}^{i, l} Y_{s}^{j, k}+X_{s}^{i} Y_{s}^{i, k, l}\right) \mathbb{Z}_{s t}^{l, k}
$$

respectively. Since $\Xi_{s t}^{i, j}-X_{s}^{i} \Xi_{s t}^{j}=Y_{s}^{i, l} Y_{s}^{j, k} \mathbb{Z}_{s t}^{l, k}$ and by definition of $X$ we get

$$
\left|\mathbb{X}_{s t}^{i, j}-Y_{s}^{i, l} Y_{s}^{j, k} \mathbb{Z}_{s t}^{l, k}\right| \lesssim|t-s|^{3 \alpha}, \quad\left|X_{s t}^{i}-Y_{s}^{i, k} Z_{s t}^{k}\right| \lesssim|t-s|^{2 \alpha}
$$

which gives

$$
\left|\mathbb{X}_{s t}^{i, j}+\mathbb{X}_{s t}^{j, i}-X_{s t}^{i} X_{s t}^{j}\right| \lesssim\left|Y_{s}^{i, l} Y_{s}^{j, k} \mathbb{Z}_{s t}^{l, k}+Y_{s}^{j, l} Y_{s}^{i, k} \mathbb{Z}_{s t}^{l, k}-Y_{s}^{j, k} Z_{s t}^{k} Y_{s}^{j, l} Z_{s t}^{l}\right|+|t-s|^{3 \alpha} .
$$

Now, since $\mathbf{Z}$ is weakly geometric we have

$$
Y_{s}^{i, l} Y_{s}^{j, k} \mathbb{Z}_{s t}^{l, k}+Y_{s}^{j, l} Y_{s}^{i, k} \mathbb{Z}_{s t}^{l, k}=Y_{s}^{i, l} Y_{s}^{j, k}\left(\mathbb{Z}_{s t}^{l, k}+\mathbb{Z}_{s t}^{k, l}\right)=Y_{s}^{i, l} Y_{s}^{j, k} Z_{s t}^{l} Z_{s t}^{k}
$$

which gives

$$
\left|\mathbb{X}_{s t}^{i, j}+\mathbb{X}_{s t}^{j, i}-X_{s t}^{i} X_{s t}^{j}\right| \lesssim|t-s|^{3 \alpha}
$$

It is straightforward to check that the above left hand side is the increment from $s$ to $t$ of the function $t \mapsto \mathbb{X}_{0 t}^{i, j}+\mathbb{X}_{0 t}^{j, i}-X_{t}^{i} X_{t}^{j}$. Since $3 \alpha>1$ we get that this function is constant and equal to 0 .

In the next lemma we show how to construct the approximation in Proposition 7.3 .

Lemma A.3. Fix $N, K, d, m>0, \bar{\alpha} \in\left(\frac{1}{3}, \frac{1}{2}\right)$ and let $\mathbf{Z} \in \mathscr{C}_{w g}^{\bar{\alpha}}\left([0, T] ; \mathbb{R}^{m}\right)$ be a weakly geometric rough path. Moreover, for $i=1, \ldots, d, n=1, \ldots, N$ and $k=1, \ldots, K$, let $e_{n} \in L^{2}\left(\mathbb{R}^{d}\right)$ be an orthonormal basis and $\theta^{i, k, n} \in \mathscr{D}_{Z}^{2 \alpha^{\prime}}([0, T], \mathbb{R})$, for $\alpha^{\prime} \in\left(\frac{1}{3}, \bar{\alpha}\right)$. Let $\phi=\phi^{i, k}=\sum_{n=1}^{N} \theta^{i, k, n} e_{n}$ and construct $\mathbf{X}^{\phi}$ as in (93). Then, for every $\alpha \in\left(\frac{1}{3}, \alpha^{\prime}\right)$ there exists $\mathbf{X}^{\epsilon}$ such that

$$
\varrho_{\alpha}\left(\mathbf{X}^{\phi}, \mathbf{X}^{\epsilon}\right) \rightarrow 0, \quad \text { for } \epsilon \rightarrow 0 .
$$

Proof. We take $\left(\bar{e}_{i}\right)_{i=1, \ldots, d}$ an orthonormal basis of $\mathbb{R}^{d}$ and, for $\bar{\imath}=1, \ldots, d N$, we define $\xi^{\bar{l}}:=e_{n} \bar{e}_{i} \in$ $L^{2}\left(\mathbb{R}^{d} ; \mathbb{R}^{d}\right)$, where $\bar{\imath}, i, n$ satisfy the relation

$$
\bar{\iota}:=d(n-1)+i, \quad i=1, \ldots, d, n=1, \ldots, N .
$$

Let $V^{N}$ be the finite dimensional vector space defined as

$$
V^{N}:=\operatorname{span}\left\{\xi^{\bar{l}} \mid \bar{\imath}=1, \ldots, d N\right\} \subset L^{2}\left(\mathbb{R}^{d} ; \mathbb{R}^{d}\right) .
$$


We note that $\operatorname{dim}\left(V^{N}\right)=d N$. On this space we construct a rough path as follows, for $\bar{\imath}, \bar{\jmath}=1, \ldots, d N$,

$$
X_{s t}^{\bar{\imath}}:=C_{s t}^{\bar{l}} \xi^{\bar{l}}:=\left(\int_{s}^{t} \theta_{r}^{i, k, n} d \mathbf{Z}_{r}^{k}\right) \xi^{\bar{l}}, \quad \mathbb{X}_{s t}^{\bar{i} \bar{\jmath}}:=C_{s t}^{\bar{l} \bar{\jmath}} \xi^{\bar{l}} \otimes \xi^{\bar{\jmath}}:=\left(\int_{s}^{t} \theta_{r}^{i, k, n} \int_{s}^{u} \theta_{u}^{j, l, m} d \mathbf{Z}_{u}^{l} d \mathbf{Z}_{r}^{k}\right) \xi^{\bar{l}} \otimes \xi^{\bar{J}} .
$$

Here and in the following we always assume that the triples $(\bar{l}, i, n)$ and $(\bar{\jmath}, j, m)$ satisfy relation (95). Moreover, we always use the convention that we are summing over repeated indices, in this case $k, l=1, \ldots, K$. It is immediate to see that $\mathbf{X}^{\phi}=\left(\sum_{\bar{l}=1}^{d N} X^{\bar{\imath}}, \sum_{\bar{l}, \bar{j}=1}^{d N} \mathbb{X}^{\bar{i}, \bar{j}}\right)$.

We prove now that $(X, \mathbb{X})$ is geometric, i.e. that the following relation holds

$$
2 \operatorname{Sym}(\mathbb{X})_{s t}=X_{s t} \otimes X_{s t}, \quad \forall s, t \in[0, T] .
$$

Let us look more in detail what the tensor product on the right hand side is, for $\bar{\imath}, \bar{j}=1, \ldots, d N$,

$$
\left(X_{s t} \otimes X_{s t}\right)^{\bar{l}, \bar{j}}=\left(C_{s t}^{\bar{l}} C_{s t}^{\bar{j}}\right) \xi^{\bar{l}} \otimes \xi^{\bar{j}}=\left(C_{s t}^{\bar{l}} C_{s t}^{\bar{j}} e_{n} e_{m}\right) \bar{e}_{i} \otimes \bar{e}_{j}
$$

Each of these terms is a tensor product which is mostly zero. Let us now describe each component of (96). We start by introducing the indexes

$$
\hat{\imath}:=d(\bar{\imath}-1)+f, \quad f=1, \ldots, d, \bar{\imath}=1, \ldots, d N .
$$

We assume from now that the couple $(l, f)$ and $(\jmath, g)$ always assume the previous relation. We obtain

$$
\left(X_{s t} \otimes X_{s t}\right)^{\hat{\imath}, \hat{\jmath}}=\left(\left(X_{s t} \otimes X_{s t}\right)^{\bar{l}, \bar{J}}\right)^{f, g}=C_{s t}^{\bar{\imath}} C_{s t}^{\bar{J}} e^{n} e^{m} \delta_{i, j, f, g} .
$$

Similarly, we see that

$$
\left(\operatorname{Sym}(\mathbb{X})_{s t}\right)^{\hat{\imath}, \hat{\jmath}}=\mathbb{X}_{s t}^{\hat{\imath}, \hat{\jmath}}+\mathbb{X}_{s t}^{\hat{\jmath}, \hat{l}}=\left(C_{s t}^{\bar{l}, \bar{j}} e^{n} e^{m}\right)\left(e_{i} \otimes e_{j}\right)^{f, g}=C_{s t}^{\bar{l}, \bar{j}} e^{n} e^{m} \delta_{i, j, f, g} .
$$

The symmetry condition reduces to verify the scalar equality

$$
C_{s t}^{\bar{l}} C_{s t}^{\bar{j}}=C_{s t}^{\bar{l}, \bar{j}},
$$

which is satisfied thanks to Lemma A.2,

The rough path $\mathbf{X}^{\phi}$ is thus in $\mathscr{C}_{w g}^{\alpha^{\prime}}\left([0, T], V^{N}\right)$. Since $V^{N}$ is a finite dimensional space, we can find a smooth approximation $\mathbf{X}^{\epsilon}$ in $\mathscr{C}^{\alpha}\left([0, T], V^{N}\right)$, for some $\alpha \in\left(\frac{1}{3}, \alpha^{\prime}\right)$. Hence, since $V^{N} \subset L^{2}\left(\mathbb{R}^{d} ; \mathbb{R}^{d}\right)$, this is also an approximation in $\mathscr{C}^{\alpha}\left([0, T], L^{2}\left(\mathbb{R}^{d} ; \mathbb{R}^{d}\right)\right)$.

\section{A.3 A separable subspace of the Hölder space}

Proposition A.4. The space $C_{0}^{\alpha}([0, T] ; E)$ is equal to the closure of $C^{1}([0, T] ; E)$ with respect to the $C^{\alpha}$-topology. In particular, $C_{0}^{\alpha}([0, T] ; E)$ is separable if $E$ is separable.

Proof. For simplicity we assume $E=\mathbb{R}$. We clearly have $[f]_{\alpha, h} \leq h^{1-\alpha}\|\nabla f\|_{\infty}$ so that $C^{1}([0, T]) \subset$ $C_{0}^{\alpha}([0, T])$, which shows one inclusion by taking the closure.

To see the reversed inclusion, we take $f \in C_{0}^{\alpha}([0, T])$, a standard mollifier $\rho_{n}(u)=n \rho(n u)$ and let $f_{t}^{n}=\int_{0}^{T} f_{u} \rho_{n}(t-u) d u=\int_{t}^{T-t} f_{t-u} \rho_{n}(u) d u$. Then $f^{n}$ is smooth and we get for $|t-s| \leq h$

$$
\left|f_{t}^{n}-f_{s}^{n}\right| \leq \int_{t}^{T-t}\left|f_{t-u}-f_{s-u}\right| \rho_{n}(u) d u \leq[f]_{\alpha, h}|t-s|^{\alpha}
$$


so that $\left[f^{n}\right]_{\alpha, h} \leq[f]_{\alpha, h}$. Let us show that $f^{n}$ converges uniformly to $f$.

$$
\begin{aligned}
\left|f_{t}-f_{t}^{n}\right| & =\left|f_{t} \int_{0}^{T} \rho_{n}(t-u) d u-\int_{0}^{T} f_{u} \rho_{n}(t-u) d u\right| \leq[f]_{\alpha} \int_{0}^{T}|t-u|^{\alpha} \rho_{n}(t-u) d u \\
& \leq[f]_{\alpha} \int_{\mathbb{R}}|t-u|^{\alpha} \rho_{n}(t-u) d u=[f]_{\alpha} n^{-\alpha} \int_{\mathbb{R}}|r|^{\alpha} \rho(r) d r
\end{aligned}
$$

which converges to 0 uniformly in $t$.

Now, write

$$
\left[f-f^{n}\right]_{\alpha} \leq\left[f-f^{n}\right]_{\alpha, h}+\sup _{(s, t) \in \Delta_{T}:|t-s| \geq h} \frac{\left|\delta f_{s t}-\delta f_{s t}^{n}\right|}{|t-s|^{\alpha}} \leq 2[f]_{\alpha, h}+2 h^{-\alpha}\left\|f-f^{n}\right\|_{\infty}
$$

which gives

$$
\lim _{n \rightarrow \infty}\left[f-f^{n}\right]_{\alpha} \leq 2[f]_{\alpha, h}
$$

By assumption on $f$, letting $h \rightarrow 0$ gives that $f^{n} \rightarrow f$ in $C^{\alpha}([0, T])$.

\section{References}

[1] I. Bailleul, R. Catellier, and F. Delarue. Mean field rough differential equations. arXiv preprint arXiv:1802.05882, 2018.

[2] I. Bailleul and M. Gubinelli. Unbounded rough drivers. Annales de la Faculté des Sciences de Toulouse. Mathématiques., 26(4):795-830, 2017.

[3] I. Bailleul and S. Riedel. Rough flows. To appear in Journal of the Mathematical Society of Japan, arXiv preprint: https://arxiv.org/abs/1505.01692, 2018.

[4] H. Brezis. Functional analysis, Sobolev spaces and partial differential equations. Universitext. Springer, New York, 2011.

[5] R. Carmona, F. Delarue, and D. Lacker. Mean field games with common noise. Ann. Probab., 44(6):3740-3803, 112016.

[6] T. Cass, C. Litterer, and T. Lyons. Integrability and tail estimates for gaussian rough differential equations. Ann. Probab., 41(4):3026-3050, 072013.

[7] T. Cass and T. Lyons. Evolving communities with individual preferences. Proc. Lond. Math. Soc. (3), 110(1):83-107, 2015.

[8] M. Coghi and B. Gess. Stochastic nonlinear fokker-planck equations. arXiv preprint arXiv:1904.07894, 2019.

[9] A. Davie. Differential equations driven by rough paths: an approach via discrete approximation. Appl. Math. Res. eXpress 35, 2010.

[10] D. Dawson and J. Vaillancourt. Stochastic McKean-Vlasov equations. Nonlinear Differential Equations and Applications NoDEA, 2(2):199-229, June 1995. 
[11] A. Deya, M. Gubinelli, M. Hofmanová, and S. Tindel. A priori estimates for rough PDEs with application to rough conservation laws. arXiv preprint arXiv:1604.00437, 2016.

[12] J. Diehl, P. K. Friz, and W. Stannat. Stochastic partial differential equations: a rough path view. Annales de la Faculté des Sciences de Toulouse. Mathématiques., 26(4), 2017.

[13] J. Diehl, H. Oberhauser, and S. Riedel. A levy-area between brownian motion and rough paths with applications to robust non-linear filtering and rpdes. Stochastic Processes and Their Applications, Volume 125, Issue 1, January 2015, Pages 161 - 181, 2015.

[14] P. Friz, T. Nilssen, and W. Stannat. Existence, uniqueness and stability of semi-linear rough partial differential equations. arXiv preprint arXiv:1809.00841, 2018.

[15] P. K. Friz and M. Hairer. A course on rough paths. Universitext. Springer, Cham, 2014. With an introduction to regularity structures.

[16] P. K. Friz and N. B. Victoir. Multidimensional Stochastic Processes as Rough Paths: Theory and Applications. Cambridge Studies in Advanced Mathematics. Cambridge University Press, 2010.

[17] M. Gubinelli. Controlling rough paths. Journal of Functional Analysis, 216(1):86 - 140, 2004.

[18] A. Hocquet and M. Hofmanová. An energy method for rough partial differential equations. Journal of Differential Equations, 265(4):1407-1466, 2018.

[19] H. Kunita. Stochastic flows and stochastic differential equations, volume 24 of Cambridge Studies in Advanced Mathematics. Cambridge University Press, Cambridge, 1990.

[20] T. G. Kurtz and J. Xiong. Particle representations for a class of nonlinear spdes. Stochastic Processes and their Applications, 83(1):103-126, 1999.

[21] T. G. Kurtz and J. Xiong. Numerical solutions for a class of SPDEs with application to filtering. In Stochastics in finite and infinite dimensions, Trends Math., pages 233-258. Birkhäuser Boston, Boston, MA, 2001.

[22] W. Liu and M. Röckner. Stochastic partial differential equations: an introduction. Universitext. Springer, Cham, 2015.

[23] D. Nualart and P. Xia. On nonlinear rough paths. arXiv preprint arXiv:1904.11526, 2019. 